\title{
ON THE CONJECTURE OF LEHMER, LIMIT MAHLER MEASURE OF TRINOMIALS AND ASYMPTOTIC EXPANSIONS
}

\author{
JEAN-LOUIS VERGER-GAUGRY
}

Dedicated to the memory of Professor Pierre Liardet.

\begin{abstract}
Let $n \geq 2$ be an integer and denote by $\theta_{n}$ the real root in $(0,1)$ of the trinomial $G_{n}(X)=-1+X+X^{n}$. The sequence of Perron numbers $\left(\theta_{n}^{-1}\right)_{n \geq 2}$ tends to 1 . We prove that the Conjecture of Lehmer is true for $\left\{\theta_{n}^{-1} \mid\right.$ $n \geq 2\}$ by the direct method of Poincaré asymptotic expansions (divergent formal series of functions) of the roots $\theta_{n}, z_{j, n}$, of $G_{n}(X)$ lying in $|z|<1$, as a function of $n, j$ only. This method, not yet applied to Lehmer's problem up to the knowledge of the author, is successfully introduced here. It first gives the asymptotic expansion of the Mahler measures $\mathrm{M}\left(G_{n}\right)=\mathrm{M}\left(\theta_{n}\right)=\mathrm{M}\left(\theta_{n}^{-1}\right)$ of the trinomials $G_{n}$ as a function of $n$ only, without invoking Smyth's Theorem, and their unique limit point above the smallest Pisot number. Comparison is made with Smyth's, Boyd's and Flammang's previous results. By this method we obtain a direct proof that the conjecture of Schinzel-Zassenhaus is true for $\left\{\theta_{n}^{-1} \mid n \geq 2\right\}$, with a minoration of the house $\theta_{n}^{-1}$, and a minoration of the Mahler measure $\mathrm{M}\left(G_{n}\right)$ better than Dobrowolski's one. The angular regularity of the roots of $G_{n}$, near the unit circle, and limit equidistribution of the conjugates, for $n$ tending to infinity (in the sense of Bilu, Petsche, Pritsker), towards the Haar measure on the unit circle, are described in the context of the Erdős-Turán-Amoroso-Mignotte theory, with uniformly bounded discrepancy functions.
\end{abstract}

\section{Communicated by Werner Georg Nowak}

2010 Mathematics Subject Classification: 11C08, 11G50, 11K16, 11K26, 11K38, 11Q05, 11R06, 11R09, 30B10, 30C15.

Keyw ords: Mahler measure, trinomial, Lehmer Conjecture, asymptotic expansion, divergent series, Perron number, Pisot number, Schinzel-Zassenhaus conjecture, Smyth conjecture, Lind-Boyd Conjecture, Boyd Conjecture, Erdős-Turán-Mignotte-Amoroso, discrepancy function, limit equidistribution. 


\section{JEAN-LOUIS VERGER-GAUGRY}

\section{Introduction}

We consider the family $\left(G_{n}(X)\right)_{n>2}$ of trinomials $G_{n}(X)=X^{n}+X-1$ and their reciprocal polynomials $G_{n}^{*}(X)=X^{n} G_{n}\left(X^{-1}\right)=-\left(X^{n}-X^{n-1}-1\right)$. Let $\theta_{n}, n \geq 2$, be the unique real root in $(0,1)$ of the trinomial $G_{n}(X)=-1+X+X^{n}$. By Smyth's Theorem [Sy1] (Dubickas [Ds1]), since the trinomials $G_{n}(X)$ are not reciprocal, the Mahler measure of $G_{n}$ satisfies

$$
\mathrm{M}\left(\theta_{n}\right)=\mathrm{M}\left(G_{n}\right) \geq \Theta=1.3247 \ldots, \quad n \geq 2,
$$

where $\Theta=\theta_{5}^{-1}$ is the smallest Pisot number, dominant root of the Pisot polynomial $X^{3}-X-1=-G_{5}^{*}(X) /\left(X^{2}-X+1\right)$. Lehmer's Conjecture [Le] (Smyth [Sy3]) asserts that there exists a constant $c>0$ such that $\mathrm{M}(\alpha) \geq 1+c$ for every nonzero algebraic number $\alpha$ which is not a root of unity (Blansky and Montgomery [ByM], Dobrowolsky [Do2], Stewart [St]). Since the sequence of Perron numbers $\theta_{n}^{-1}, n \geq 2$, tends to 1 when $n$ tends to infinity (Lemma 2.2 ), the inequalities (1.1) imply that Lehmer's Conjecture is true for the family $\left\{\theta_{n}^{-1} \mid n \geq 2\right\}$ with $c=\Theta-1$.

Lehmer's problem has a long history (Smyth [Sy3]). The search of a proof of Lehmer's Conjecture has led many authors to develop various technics for obtaining a minoration of the Mahler measure, or equivalently the Weil height in the classical context, or in a generalized context: e.g., polynomials (Amoroso [A1], Borwein, Dobrowolski and Mossinghoff [BDM], Flammang [F], Rhin and Smyth [RS], Rhin and Wu [RW], Silverman [Sn]), multivariate polynomials (Boyd [Bo2], Schinzel [Sc3], Smyth [Sy2]), algebraic numbers (Louboutin [Lt]), in abelian extensions (Amoroso and Dvornicich [AD], Amoroso and Zannier [AZ2], Baker [Bk]), limit points (Bertin et al [B-S], Boyd and Mossinghoff [BM], Flammand, Grandcolas and Rhin [FGR]), using auxiliary functions and interpolation determinants (Cantor and Strauss [CS], Laurent [La2], Stewart [St], Waldschmidt [W2]), elliptic curves and elliptic Mahler measures (Galateau and Mahé [GM], Hindry and Silverman [HS], Laurent [La], Masser [Ma], Ratazzi $[\mathrm{Rz}]$ ), abelian varieties (David and Hindry [DH], Rémond [Rd]), p-adic Mahler measures (Besser and Deninger $[\mathrm{BD}]$ ).

The objective of this note is to give a direct proof of Lehmer's Conjecture for the family $\left\{\theta_{n}^{-1} \mid n \geq 2\right\}$ by using the (Poincaré) asymptotic expansions of the roots $\theta_{n}, z_{j, n}$, of $G_{n}, n \geq 2$ (Borel [Bl], Copson [C], Dingle [Di], Erdélyi [E]), as a function of $n, j$ only (cf. Section 3 ). Once these asymptotic expansions obtained, other conjectures can readily be investigated (Section 5): (i) we will consider Smyth conjecture for the trinomials $G_{n}$ (of height 1 ) of small Mahler measure, 
(ii) we will obtain an effective minoration of the Mahler measures $\mathrm{M}\left(G_{n}\right)$, and (iii) we will give a proof of Schinzel-Zassenhaus conjecture for $\left\{\theta_{n}^{-1} \mid n \geq 2\right\}$ with an explicit value of the constant in the minoration of the house $\left|\theta_{n}^{-1}\right|=\theta_{n}^{-1}$.

Namely, we write

$$
\begin{gathered}
\theta_{n}=\mathrm{D}\left(\theta_{n}\right)+\operatorname{tl}\left(\theta_{n}\right), \\
\operatorname{Re}\left(z_{j, n}\right)=\mathrm{D}\left(\operatorname{Re}\left(z_{j, n}\right)\right)+\operatorname{tl}\left(\operatorname{Re}\left(z_{j, n}\right)\right), \\
\operatorname{Im}\left(z_{j, n}\right)=\mathrm{D}\left(\operatorname{Im}\left(z_{j, n}\right)\right)+\operatorname{tl}\left(\operatorname{Im}\left(z_{j, n}\right)\right),
\end{gathered}
$$

where "D" stands for "development" (or "limited expansion", or "lowest order terms") and "tl" for "tail" (or "remainder", or "terminant" in [Di]), and consider the products

$$
\Pi_{G_{n}}:=\mathrm{D}\left(\mathrm{M}\left(G_{n}\right)\right)=\mathrm{D}\left(\theta_{n}\right)^{-1} \times \prod_{z_{j, n}} \prod_{\text {in }|z|<1} \mathrm{D}\left(\left|z_{j, n}\right|\right)^{-2}
$$

instead of $\mathrm{M}\left(G_{n}\right)$, as approximant value of $\mathrm{M}\left(G_{n}\right)$. Ideally each quantity should be represented by " $D$ " as an infinite sum, of infinitely many terms, for an exact matching, and then with a tail "tl" equal to zero; but, in the way they are obtained here (cf. in the proofs of Proposition 3.1 and Proposition 3.4), these terms "D", if they were infinite, as asymptotic expansions of the variables $n$ and $j$, were divergent expansions. Divergence would be a problem. But the terminants are readily obtained by the method; to overcome the difficulty of divergence, the objective is to show that the control of the terminants (Dingle [Di] Chap. XXI and XXII, "Theory of Terminants") at a sufficiently high order, but not too high, makes sense.

Proving Lehmer's Conjecture for $\left\{\theta_{n}^{-1} \mid n \geq 2\right\}$ amounts to (i) show that the limited expansions $\mathrm{D}\left(\theta_{n}\right), \mathrm{D}\left(\operatorname{Re}\left(z_{j, n}\right)\right)$ and $\mathrm{D}\left(\operatorname{Im}\left(z_{j, n}\right)\right)$ can be "given a push" up till an order (i.e., can be represented as sums of a sufficiently high number of lower order terms) for which the tails

$$
\mathrm{tl}_{G_{n}}:=\mathrm{M}\left(G_{n}\right)-\Pi_{G_{n}}
$$

satisfy $\lim _{n \rightarrow+\infty} \mathrm{tl}_{G_{n}}=0$, (ii) give an uniform lower bound $>1$ to the approximant values $\Pi_{G_{n}}$. We will show more, namely that the two limits

$$
\lim _{n \rightarrow+\infty} \Pi_{G_{n}} \text { and } \lim _{n \rightarrow+\infty} \mathrm{M}\left(G_{n}\right)
$$

exist, are equal and greater than $\Theta$.

Roughly speaking the $n$ roots of $G_{n}$ are considered as $n$ "bodies" as in celestial mechanics (in the so-called " $n$-body problem"), not linked by differential equations of movement but only by the polynomial equation $G_{n}(z)=0$; and we mimic here the strategy of $\mathrm{H}$. Poincaré $[\mathrm{P}]$ to describe them in an " $n$-body problem" using a sort or "theory of perturbations", by divergent asymptotic expansions. It seems that this strategy has never been introduced as such, though divergent 
formal series were already shown to play a major role into many problems [Bl]: cf. the remarkable book "Divergent Series" by G. H. Hardy for instance.

This method of Poincaré asymptotic expansions is a direct attack of the conjectures (Lehmer, Schinzel-Zassenhaus), which does not make use of Smyth's Theorem (Amoroso [A2]). It amounts to study the geometry of the set of values of Mahler measures $\mathrm{M}\left(G_{n}\right)$ and its limit points (Amoroso [A1], Boyd and Mossinghoff [BM], Dixon and Dubickas [DDs], Langevin [Lg], Smyth [Sy3]) by "controlled" approximants. Theorem 1.1 is obtained by this method of asymptotic expansions (in Section 4.2) and shows that the first derived set of $\left\{\mathrm{M}\left(G_{n}\right) \mid n \geq 2\right\}$ is reduced to one element, $1.38135 \ldots$

To confirm the relevance of the present method of asymptotic expansions, with a control of the tails of the expansions, we prove Theorem 1.1 in two ways: by this method (Section 4.2) and by using a method initiated by Boyd with bivariate Mahler measures (Section 4.1). In both cases, we obtain the same value of the limit, called the limit Mahler measure of the trinomials $G_{n}$.

TheOREM 1.1. Let $\chi_{3}$ be the uniquely specified odd character of conductor 3 $\left(\chi_{3}(m)=0,1\right.$ or -1 according to whether $m \equiv 0,1$ or $2(\bmod 3)$, equivalently $\chi_{3}(m)=\left(\frac{m}{3}\right)$ the Jacobi symbol), and denote $L\left(s, \chi_{3}\right)=\sum_{m \geq 1} \frac{\chi_{3}(m)}{m^{s}}$ the Dirichlet L-series for the character $\chi_{3}$. Then

$$
\begin{aligned}
\lim _{n \rightarrow+\infty} \mathrm{M}\left(G_{n}\right) & =\exp \left(\frac{3 \sqrt{3}}{4 \pi} \mathrm{L}\left(2, \chi_{3}\right)\right) \\
& =\exp \left(\frac{-1}{\pi} \int_{0}^{\pi / 3} \log \left(2 \sin \left(\frac{x}{2}\right)\right) \mathrm{d} x\right)=1.38135 \ldots=: \Lambda .
\end{aligned}
$$

Whether $\Lambda$ is algebraic or transcendental is unkown (Boyd [Bo1]). Another question of Boyd [Bo0] is whether $\Lambda$ belongs to the second derived set of the set $\{\mathrm{M}(\beta)\}$ of Mahler measures of algebraic numbers. The value $\log \Lambda$, as Clausen's integral, is obtained by the Bloch-Wigner dilogarithm [BM] [Ln]. In Section 5.1 we obtain the following asymptotic expansions of $\mathrm{M}\left(G_{n}\right)$ in a neighbourhood of the limit Mahler measure $\Lambda=1.38135 \ldots$ of Theorem 1.1.

Theorem 1.2. Let $n_{0}$ be an integer such that $\frac{\pi}{3}>2 \pi \frac{\log n_{0}}{n_{0}}$, and let $n \geq n_{0}$. Then

$$
\mathrm{M}\left(G_{n}\right)=\left(\lim _{m \rightarrow+\infty} \mathrm{M}\left(G_{m}\right)\right)\left(1+r(n) \frac{1}{\log n}+O\left(\frac{\log \log n}{\log n}\right)^{2}\right)
$$

with the constant $1 / 6$ involved in the Big $O$, and with $r(n)$ real, $|r(n)| \leq 1 / 6$. 


\section{CONJECTURE OF LEHMER, ASYMPTOTIC EXPANSIONS OF THE MAHLER MEASURE}

In the sequel, we take $n_{0}=18$. Whether the coefficients $r(n), n \geq n_{0}$ are negative or positive they would allow to prove Smyth's Conjecture for the trinomials $G_{n}$; we recall Smyth's Conjecture below, for general trinomials of (naïve) height 1 (Flammang $[\mathrm{F}]$ ). However the coefficients $r(n)$ do not seem to be easily computed by this method. We report in Theorem 1.3 the distribution of the values $\mathrm{M}\left(G_{n}\right)$ in a neighbourhood of $\Lambda$ provided by the original method introduced by Smyth and Boyd in [Bo3], in which the signs of the coefficients $s(n)$ of the second-order terms are accessible and computed.

ConjeCture (Smyth). For all integers $n \geq 4, k \geq 1$ such that

$$
\operatorname{gcd}(n, k)=1, \quad k<n / 2,
$$

- $\mathrm{M}\left(z^{n}+z^{k}+1\right)<\Lambda$ if and only if 3 divides $n+k$,

- $\mathrm{M}\left(z^{n}-z^{k}+1\right)<\Lambda$ with $n$ odd if and only if 3 does not divide $n+k$,

- $\mathrm{M}\left(z^{n}-z^{k}-1\right)<\Lambda$ with $n$ even if and only if 3 does not divide $n+k$.

Smyth's conjecture was recently proved by Flammang $[\mathrm{F}]$ for large $n$, using similar techniques; see also Borwein and Straub [BS]. Even though the Lerch transcendent function (or other transcendental functions) is used in the proof of Theorem 1.3, the values $\mathrm{M}\left(G_{n}\right)$ are all Perron numbers (Adler and Marcus [AM], Dubickas [Ds5]).

Theorem 1.3 is obtained by a method dedicated to the trinomials $G_{n}$ while Theorem 1.2, obtained by a totally different type of proof, independently, can be generalized to other families of polynomials than $\left\{G_{n}\right\}$.

Theorem 1.3. Let $n \geq 2$ be an integer. Then

$$
\mathrm{M}\left(-1+X+X^{n}\right)=\left(\lim _{m \rightarrow+\infty} \mathrm{M}\left(G_{m}\right)\right)\left(1+\frac{s(n)}{n^{2}}+O\left(n^{-3}\right)\right)
$$

with, for $n$ odd:

$$
s(n)=\left\{\begin{aligned}
\sqrt{3} \pi / 18 & =+0.3023 \ldots & & \text { if } n \equiv 1 \text { or } 3 \\
-\sqrt{3} \pi / 6 & =-0.9069 \ldots & & \text { if } n \equiv 5
\end{aligned}\right.
$$

for $n$ even:

$$
s(n)=\left\{\begin{array}{llrl}
-\sqrt{3} \pi / 36 & =-0.1511 \ldots & & \text { if } n \equiv 0 \text { or } 4 \\
+\sqrt{3} \pi / 12=+0.4534 \ldots & & \text { if } n \equiv 2 & (\bmod 6),
\end{array}\right.
$$

In the search of smallest limit Mahler measures, Smyth [Sy4] obtained that the set of Mahler measures $\{\mathrm{M}(\alpha) \mid \alpha \neq 0$ nonreciprocal algebraic number $\}$ admits $\Theta$ as isolated infimum. Comparatively, the distribution of values of Mahler's measure for polynomials having coefficients in $\mathbb{Z}$ of bounded Mahler measure is in general spread over larger intervals than a neighbourhood of an unique 
limit point (Chern and Vaaler [CV], Dixon and Dubickas [DDs], Sinclair [Si]). Corollary 1.4, obtained by Smyth and Boyd's method i.e., as deduced from Theorem 1.3, or by the method of asymptotic expansions, shows that the constant of Lehmer $c=\Theta-1$ is reached only once.

Corollary 1.4. $\mathrm{M}\left(G_{2}\right)=\theta_{2}^{-1}=\frac{1+\sqrt{5}}{2}=1.618 \ldots, \mathrm{M}\left(G_{n}\right) \geq \mathrm{M}\left(G_{5}\right)=\theta_{5}^{-1}=\Theta$ for all $n \geq 3$, with equality if and only if $n=5$.

Corollary 1.5. Smyth's conjecture is true for $\left\{G_{n} \mid n \geq 4\right\}$.

The method of asymptotic expansions does not seem to be powerful enough for solving Smyth's conjecture (i.e., Flammang's Theorem) for $k=1$, neither probably for the generic three cases " $n, k$ " in its statement (covering all cases of trinomials of height 1). Comparing (1.3) and (1.4), these two methods surprisingly give rise to very different speeds of convergence towards the limit, simply meaning that (1.3) underestimates it.

However the method of asymptotic expansions gives a new insight into the problem of the minoration of the Mahler measure $\mathrm{M}\left(G_{n}\right)$. In 1979 Dobrowolski [Do2] proved that

$$
\mathrm{M}(\alpha)>1+(1-\epsilon)\left(\frac{\log \log d}{\log d}\right)^{3}, \quad d>d_{1}(\epsilon) .
$$

for any nonzero algebraic number $\alpha$ of degree $d$. From Theorem 1.2 we obtain the following minoration of $\mathrm{M}\left(\theta_{n}^{-1}\right)$ which is better than (1.5).

Corollary 1.6.

$$
\mathrm{M}\left(\theta_{n}^{-1}\right)>\Lambda-\frac{\Lambda}{6}\left(\frac{1}{\log n}\right), \quad n \geq n_{1}=2 .
$$

The value of $n_{1}$ is effective: for the small values of $n$, a numerical comparison between Theorem 1.3 and the inequality (1.6) gives $n_{1}=2$. In (1.6) the exponent of the denominator " $\log n$ " is now " 1 " instead of " 3 ", where $n=\operatorname{deg} \theta_{n}^{-1}$ if $n \not \equiv 5 \quad(\bmod 6)$, and $n=\operatorname{deg} \theta_{n}^{-1}+2$ if $n \equiv 5 \quad(\bmod 6)$. That $n$ is closely related to the degree, but different in some cases, is common: quoting Waldschmidt in [W2, p. 90], "we insist that $n$ is only an upper bound for the degree of $\theta_{n}^{-1}$, and not the actual degree". Minorations of the Mahler measure obtained by several authors (Waldschmidt [W2, §3.6]) are discussed in Section 5.2.

Assume that $\mathbb{L}$ is a totally real algebraic number field, or a CM field (a totally complex quadratic extension of a totally real number field); then for any nonzero algebraic integer $\alpha \in \mathbb{L}$, of degree $d$, not being a root of unity, Schinzel [Sc2] obtained the minoration

$$
\mathrm{M}(\alpha) \geq \theta_{2}^{-d / 2}
$$


Here, from Theorem 1.2, the following theorem of Zagier [Za] which claims

$$
\mathrm{M}(\alpha) \mathrm{M}(\alpha-1) \geq \theta_{2}^{-d / 2}
$$

for algebraic numbers $\alpha$, of degree $d, \alpha \neq 0, \alpha \neq 1, \alpha \neq \frac{1+\sqrt{-3}}{2}$, and the improvment of the minoration of the Zhang-Zagier height by Doche [De], we readily obtain the following minoration.

Corollary 1.7. Let $u=0$ except if $n \equiv 5 \bmod 6$ in which case $u=-2$. Then

$$
\mathrm{M}\left(\theta_{n}^{-1}-1\right) \geq \kappa \frac{\theta_{2}^{-(n+u) / 2}}{\Lambda}\left(1-\frac{1}{6 \log n}\right), \quad n \geq 2,
$$

with $\kappa=1.2817770214 / \sqrt{\theta_{2}^{-1}}=1.0076708 \ldots$

The method of asymptotic expansions also gives a direct proof of the conjecture of Schinzel-Zassenhaus (Section 5.3; Dubickas [Ds1], Schinzel-Zassenhaus [SZ]) for $\left\{\theta_{n}^{-1} \mid n \geq 2\right\}$ as follows

TheOREM 1.8. For all $n \geq 2$,

$$
\theta_{n}^{-1} \mid=\theta_{n}^{-1} \geq 1+\frac{c}{n}
$$

with $c=2\left(\theta_{2}^{-1}-1\right)=1.2360 \ldots$ reached only for $n=2$, and,

$$
\mid \theta_{n}^{-1}=\theta_{n}^{-1}>1+\frac{(\log n)\left(1-\frac{\log \log n}{\log n}\right)}{n} .
$$

The proof (Section 5.3) of Theorem 1.8 is a consequence of the asymptotic expansion of $\theta_{n}$ and does not invoke Smyth's Theorem [Sy1], which claims

$$
|\beta|>1+\frac{\log \Theta}{n}
$$

for any nonreciprocal algebraic integer $\beta$, of degree $n$. For $n$ large enough, assuming true Lind-Boyd's conjecture and Boyd's conjecture (recalled in Section 5.3), we show that $\theta_{n}^{-1}$ is a nonextremal Perron number: the minimum $\mathrm{m}_{h}(n)$ of the houses of the algebraic integers of degree $n$ is certainly smaller than the lower bound given by (1.10), and the inequality (1.10) corresponds to the asymptotic excess of $\theta_{n}^{-1}$ off extremality in some sense. Another way for obtaining a minoration of (the house of ) $\theta_{n}^{-1}$, more classical, consists in deducing it from the Mahler measure (1.6) and the inequality $\mathrm{M}\left(\theta_{n}^{-1}\right) \leq\left(\theta_{n}^{-1}\right)^{n}$; we obtain the following:

$$
\left|\theta_{n}^{-1}\right|=\theta_{n}^{-1}>1-\frac{1}{6}\left(\frac{1}{\log n}\right) \frac{1}{n}, \quad n>n_{2} .
$$

The inequality (1.11) is but less good than (1.9) and (1.10) in Theorem 1.8. 


\section{JEAN-LOUIS VERGER-GAUGRY}

In Section 6, we consider the angular distribution of the conjugates of the Perron numbers $\theta_{n}^{-1}, n \geq 2$, and their limit distribution on the unit circle.

For any Salem number $\alpha<1.3$, of degree $2 d$, of conjugates $\alpha^{(i)}$, having positive imaginary part, M.-J. Bertin $[\mathrm{Bn}]$ showed

$$
\frac{1}{d-1} \sum_{j=1}^{d-1} \arg \left(\alpha^{(j)}\right) \geq 0.96, \quad d>d_{2} .
$$

In Subsection 6.1 we show that the same type of slight angular dissymmetry may occur for the conjugates $z_{j, n}^{-1}$ of $\theta_{n}^{-1}$ which lie in the first quadrant, but in fact it disappears when $n$ tends to infinity.

TheOREM 1.9.

with

$$
\lim _{n \rightarrow+\infty} \frac{1}{\left\lfloor\frac{n-1}{4}\right\rfloor} \sum_{j=1}^{\left\lfloor\frac{n-1}{4}\right\rfloor} \arg \left(z_{j, n}\right)=\frac{\pi}{4}=0.785 \ldots,
$$

$$
\frac{1}{\left\lfloor\frac{n-1}{4}\right\rfloor} \sum_{j=1}^{\left\lfloor\frac{n-1}{4}\right\rfloor} \arg \left(z_{j, n}\right) \geq \frac{\pi}{4}-\frac{2 \pi}{n}\left(\frac{\log \log n}{\log n}\right)^{2} \quad n \geq 5 .
$$

For limit distributions of conjugates, we show that the method of bivariate Mahler measures, or equivalently the method of asymptotic expansions of the roots of $G_{n}$, as a function of $n, j$, allows to determine the limsup of Mignotte's discrepancy functions for the trinomials $G_{n}$ in the Erdös-Turán-AmorosoMignotte (ETAM) theory (Theorem 6.1). On the other hand, from Theorem 1.1 and Theorem 1.2, we deduce the asymptotic expansion of the Weil height of $\theta_{n}^{-1}$ as

$$
h\left(\theta_{n}^{-1}\right)=\frac{1}{n}\left(\frac{3 \sqrt{3}}{4 \pi} L\left(2, \chi_{3}\right)+O\left(\frac{1}{\log n}\right)\right)
$$

and therefore $\lim _{n \rightarrow \infty} h\left(\theta_{n}^{-1}\right)=0$. As a consequence the limit distribution of the conjugates of $\theta_{n}^{-1}$ is given by Theorem 6.2, as an analogue of Bilu's equidistribution Theorem of small points on the 1-dimensional torus.

Notations. Let $P(z)$ be a polynomial $\in \mathbb{Z}[z]$, and $n=\operatorname{deg}(P)$. The reciprocal polynomial of $P(z)$ is $P^{*}(z)=z^{n} P\left(\frac{1}{z}\right)$. A polynomial $P$ is a reciprocal polynomial if $P^{*}(z)=P(z)$. The opposite of the reciprocal polynomial: $-G_{n}^{*}$, of $G_{n}$, is the Parry polynomial of $\theta_{n}^{-1}$ (Verger-Gaugry [VG]). If $P(X)=a_{0} \prod_{j=1}^{n}\left(X-\alpha_{j}\right)$ is a polynomial of degree $n \geq 1$ with coefficients in $\mathbb{C}$, and roots $\alpha_{j}$, the Mahler measure of $P$ is

$$
\mathrm{M}(P):=\left|a_{0}\right| \prod_{j=1}^{n} \max \left\{1,\left|\alpha_{j}\right|\right\}
$$


The Mahler measure of an algebraic number is the Mahler measure of its minimal polynomial. Related to the Mahler measure of an algebraic integer $\alpha$ is $\mid \alpha$, the house of $\alpha$, defined as the maximum modulus of its conjugates, including $\alpha$ itself. A Perron number is either 1 or a real algebraic integer $\theta>1$ such that the Galois conjugates $\theta^{(i)}, i \neq 0$, of $\theta^{(0)}:=\theta$ satisfy: $\left|\theta^{(i)}\right|<\theta$. A Pisot number is a Perron number $>1$ for which $\left|\theta^{(i)}\right|<1$ for all $i \neq 0$.

Let $A$ be a countable subset of the line. The first derived set of $A$ is by the definition the set of the limit points of nonstationary infinite sequences of elements of $A$. The second derived set of $A$ is the first derived set of the first derived of $A$. Recall that the smallest element in the first derived set of the set of Pisot numbers is the golden mean $\theta_{2}^{-1}=(1+\sqrt{5}) / 2$ (Bertin et al. [B-S]). In particular, if $A$ is the set of Mahler measures of algebraic numbers (in the algebraic closure $\overline{\mathbb{Q}} \subset \mathbb{C}$ of $\mathbb{Q}$ ), then Lehmer's Conjecture implies that 1 does not belong to the first derived set of $A$. For $x>0, \log ^{+} x$ denotes $\max \{0, \log x\}$. Let $\mathcal{F}$ be an infinite subset of the set of nonzero algebraic numbers which are not a root of unity; we say that the Conjecture of Lehmer is true for $\mathcal{F}$ if there exists a constant $c_{\mathcal{F}}>0$ such that

$$
\mathrm{M}(\alpha) \geq 1+c_{\mathcal{F}} \quad \text { for all } \quad \alpha \in \mathcal{F} .
$$

For instance, the Conjecture of Lehmer is true for the set of Pisot numbers, and remains open for the set of Salem numbers. We use the same terminology, i.e., "true for ...", for the other conjectures: for the Conjecture of Schinzel-Zassenhaus, the Conjecture of Smyth,...

\section{Factorization of the trinomials $-1+X+X^{n}$}

The factorization of $G_{n}(X)$ is more or less known since Selmer [Sr]. For fixing the notation and a simple, convenient, and coherent indexation of the roots, it is needed under the form (2.15) in the sequel. The form (2.15) is justified below by the Lemmas 2.15, 2.2 and the Propositions 2.3, 3.1, 3.4 and 3.7. Summing in pairs over complex conjugate imaginary roots, we write the factorization of $G_{n}(X)$ as

$$
G_{n}(X)=\left(X-\theta_{n}\right)\left(\prod_{j=1}^{\left\lfloor\frac{n}{6}\right\rfloor}\left(X-z_{j, n}\right)\left(X-\overline{z_{j, n}}\right)\right) \times q_{n}(X),
$$

where $\theta_{n}$ is the only (real) root of $G_{n}(X)$ in the interval $(0,1)$, 
where

$$
q_{n}(X)= \begin{cases}\left(\prod_{j=1+\left\lfloor\frac{n}{6}\right\rfloor}\left(X-z_{j, n}\right)\left(X-\overline{z_{j, n}}\right)\right) \times\left(X-z_{\frac{n}{2}, n}\right) & \text { if } n \text { is even, with } \\ \prod_{j=1+\left\lfloor\frac{n}{6}\right\rfloor}^{\frac{n-1}{2}}\left(X-z_{j, n}\right)\left(X-\overline{z_{j, n}}\right) & z_{\frac{n}{2}, n} \text { real }<-1,\end{cases}
$$

where the index $j=1,2, \ldots$ is such that $z_{j, n}$ is a (nonreal) complex zero of $G_{n}(X)$, except if $n$ is even and $j=n / 2$, such that the $\operatorname{argument} \arg \left(z_{j, n}\right)$ of $z_{j, n}$ is roughly equal to $2 \pi j / n$ and that the family of $\operatorname{arguments}\left(\arg \left(z_{j, n}\right)\right)_{1 \leq j<\lfloor n / 2\rfloor}$ forms a strictly increasing sequence with $j$ :

$$
0<\arg \left(z_{1, n}\right)<\arg \left(z_{2, n}\right)<\cdots<\arg \left(z_{\left\lfloor\frac{n}{2}\right\rfloor, n}\right) \leq \pi .
$$

For $n \geq 2$ all the roots of $G_{n}(X)$ are simple, and the roots of $G_{n}^{*}(X)$, as inverses of the roots of $G_{n}(X)$, are classified in the reversed order (see Figure 1).

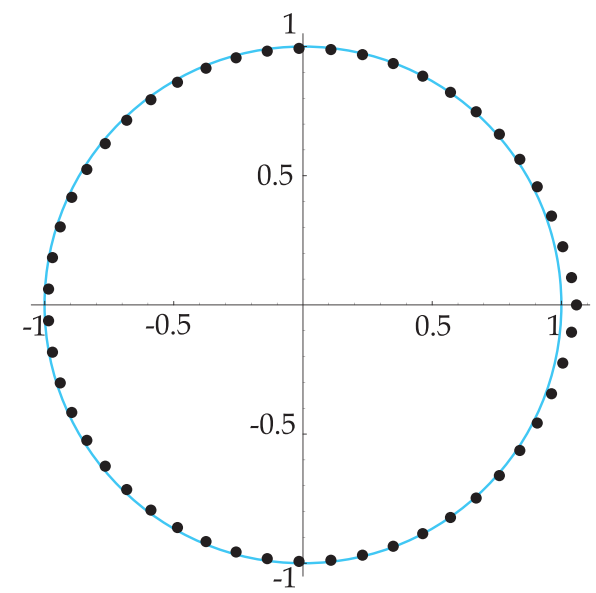

FiguRE 1. The roots (black bullets) of $G_{n}^{*}(X)$ (represented here with $n=51$ ) are uniformly distributed near $|z|=1$ according to the theory of Erdős-Turán-Amoroso-Mignotte. A slight bump appears in the half-plane $\Re(z)>1 / 2$ in the neighbourhood of 1 , at the origin of the different regimes of asymptotic expansions. The dominant root of $G_{n}^{*}(X)$ is the Perron number $\theta_{n}^{-1}>1$. 
Lemma 2.1. For all $n \geq 2$, all zeros $z_{j, n}$ and $\theta_{n}$ of the polynomials $G_{n}(X)$ have a modulus in the interval

$$
\left[1-\frac{2 \log n}{n}, 1+\frac{2 \log 2}{n}\right] .
$$

P r o of. Selmer [Sr, pp. 291-292].

The zeros of the trinomials $G_{n}(X)$ lie at a distance of the unit circle $|z|=1$ which tends to zero with $n$ tending to infinity due to the bounds (2.16). The lower bound is further improved in Proposition 3.1 and Proposition 3.4, as a function of $n$ and the index $j$ of the root only, for those roots lying in the unit disc. The coherency of the indexation of the roots of $G_{n}(X)$ is made clearer by Proposition 3.7.

Lemma 2.2. (i) The trinomial $G_{n}(X)$ admits an unique real root $\theta_{n}$ in the interval $(0,1)$. The sequence $\left(\theta_{n}\right)_{n \geq 2}$ is strictly increasing, with

$$
\theta_{2}=\frac{2}{1+\sqrt{5}}=0.618 \ldots, \quad \text { and } \lim _{n \rightarrow+\infty} \theta_{n}=1 .
$$

(ii) The root $\theta_{n}$ is the unique root of smallest modulus among all the roots of $G_{n}(X)$.

Proof. (i) For all $n \geq 2$, the derivative $G_{n}^{\prime}(x)$ is strictly positive on $[0,1]$ and the values $G_{n}(0)=-1$ and $G_{n}(1)=1$ are of opposite sign. Now $\theta_{n+1}>\theta_{n}$ since $G_{n+1}\left(\theta_{n}\right)=\left(\theta_{n}\right)^{n+1}+\theta_{n}-1=-\left(\theta_{n}-1\right)^{2}<0$. The inequalities $0<$ $\theta_{n}<\theta_{n+1}<1$ imply that the sequence $\left(\theta_{n}\right)_{n \geq 2}$ is increasing. By Lemma 2.1, using (2.16), we deduce that $\lim _{n \rightarrow+\infty} \theta_{n}=1$.

(ii) Among the moduli of the roots of $G_{n}(X) \theta_{n}$ is the smallest one: indeed, for all $n \geq 2$ and all $z$ such that $|z|<\theta_{n},\left|G_{n}(z)\right| \geq 1-|z|-|z|^{n}=-G_{n}(|z|)>0$. The uniqueness comes from Selmer [Sr, pp 291-292].

As a consequence of Lemma 2.2 the dominant root of the reciprocal polynomial $G_{n}^{*}(X)$ of $G_{n}(X), n \geq 2$, is $\theta_{n}^{-1}>1$ : the other $\operatorname{roots} z_{j, n}^{-1}, \overline{z_{j, n}^{-1}}$ of $G_{n}^{*}(X)$ satisfy $\left|z_{j, n}^{-1}\right|<\theta_{n}^{-1}$ for $j \in\left\{1,2, \ldots,\left\lfloor\frac{n}{2}\right\rfloor\right\}$. Therefore, $\theta_{n}^{-1}$ is a Perron number.

From Flatto, Lagarias and Poonen [FLP] the roots $z_{1, n}^{-1}$ and $\overline{z_{1, n}^{-1}}$ are the roots $\neq 1$ the closest to 1 and the alone second-largest roots of $G_{n}^{*}(X)$.

Proposition 3.8 extends Lemma 2.2 (ii) and [FLP] by providing a complete classification of the roots of $G_{n}$, lying in $|z|<1$, by strictly increasing modulus.

The Pisot number (golden mean) $\theta_{2}^{-1}=\frac{1+\sqrt{5}}{2}=1.618 \ldots$ is the largest Perron number in the family $\left(\theta_{n}^{-1}\right)_{n \geq 2}$. 
Proposition 2.3. Let $n \geq 2$. If $n \not \equiv 5(\bmod 6)$, then $G_{n}(X)$ is irreducible. If $n \equiv 5$ pmod6, then the polynomial $G_{n}(X)$ admits $X^{2}-X+1$ as irreducible factor in its factorization and $G_{n}(X) /\left(X^{2}-X+1\right)$ is irreducible.

P r o of. Selmer in [Sr, Theorem 3 ] shows that either $G_{n}(X)$ is irreducible, or is the product of two irreducible factors, whose one is necessarily of the type $X^{2}-X+1=\left(X-e^{i \pi / 3}\right)\left(X-e^{-i \pi / 3}\right)$. A modulus equal to 1 for the roots of $G_{n}(X)$ can occur only for $z=e^{ \pm i \pi / 3}$. It is easily seen that this is possible only in the case $n \equiv 5 \quad(\bmod 6)$.

\section{Poincaré asymptotic expansions of the roots}

In the calculation of the Mahler measure of the trinomial $G_{n}, n \geq 2$, only one part of the roots of $G_{n}$ is required, since $\mathrm{M}\left(G_{n}\right)=\mathrm{M}\left(\theta_{n}^{-1}\right)=\mathrm{M}\left(\theta_{n}\right)$ : those which lie inside the closed unit disc, or equivalently, those which lie outside the closed unit disc. We will consider those which lie inside the closed unit disc: they will be characterized by a real part greater than $1 / 2$ (Proposition 3.7). The idea followed here consists in expanding asymptotically $\theta_{n}$ and those roots $z_{j, n}$ of $G_{n}(X)$ for which $\Re\left(z_{j, n}\right)$ is strictly greater than $1 / 2$ as a function of $n$, and $j$, at an order which is sufficiently high, in order to make precise calculations with approximant values. The others roots $z_{j, n}$ for which $0<\Re\left(z_{j, n}\right) \leq 1 / 2$ will also be considered, in particular for studying how $\left\{z_{j, n}\right\}$ are dispatched in the neighbourhood of $e^{i \pi / 3}$.

The expressions of the asymptotic expansions given below come from the iterative resolution of the equation $G_{n}(z)=0$, starting from the coefficient vector $(-1,1,0,0, \ldots, 1)$ of $G_{n}$, with common height equal to 1 (for all $n \geq 2$ ), of length $n+1$ : cf. Remark 3.3 for precise statements.

The terminology order in a given asymptotic expansion comes from the general theory (Borel [Bl], Copson [C], Dingle [Di], Erdélyi [E]); the approximant solutions of $G_{n}(z)=0$ which arise naturally correspond to order 1 . The solutions corresponding to order 2 are obtained by inserting the order 1 approximant solutions into the equation $G_{n}(z)=0$, for getting order 2 approximant solutions, and so on. The order is defined as the number of steps in this iterative convergence process. Ideally, exact expressions of the roots of $G_{n}$, as (infinite) asymptotic expansions of the variables $n$ and $j$, can be obtained by iterating indefinitely this process. However, it would be a formidable task to do so, and stopping at an order sufficiently high is suitable for making calculations, increasing relevance of this iteration over a few steps. 


\section{CONJECTURE OF LEHMER, ASYMPTOTIC EXPANSIONS OF THE MAHLER MEASURE}

The theory of terminants has a long history, refers to the use of divergent series, at least since Euler, and Poincaré for the many-body problem in celestial mechanics, and to asymptotic expansions of many various types (Borel [Bl], Dingle [Di, Chap. XXI]).

For the roots of $G_{n}$ which lie in $|z|<1$, we generically write their asymptotic expansions under the following form:

$$
\begin{gathered}
\theta_{n}=\mathrm{D}\left(\theta_{n}\right)+\operatorname{tl}\left(\theta_{n}\right), \\
\operatorname{Re}\left(z_{j, n}\right)=\mathrm{D}\left(\operatorname{Re}\left(z_{j, n}\right)\right)+\operatorname{tl}\left(\operatorname{Re}\left(z_{j, n}\right)\right), \\
\operatorname{Im}\left(z_{j, n}\right)=\mathrm{D}\left(\operatorname{Im}\left(z_{j, n}\right)\right)+\operatorname{tl}\left(\operatorname{Im}\left(z_{j, n}\right)\right),
\end{gathered}
$$

where "D" and "tl" have the meaning given in the introduction. Instead of considering the "exact" product

we consider the approximant products

$$
\mathrm{M}\left(G_{n}\right)=\theta_{n}^{-1} \prod_{j=1}^{\lfloor n / 6\rfloor}\left|z_{j, n}\right|^{-2}
$$

$$
\Pi_{G_{n}}:=D\left(\theta_{n}\right)^{-1} \times \prod_{j=1}^{\lfloor n / 6\rfloor} \mathrm{D}\left(\left|z_{j, n}\right|\right)^{-2}
$$

in which the Ds' are expanded at a sufficiently high order, and where the in$\operatorname{dex} j$ allowing the enumeration of the roots, and its maximal value $\lfloor n / 6\rfloor$, is justified by Proposition 3.7. We are bound to work with expressions of $\mathrm{D}\left(\theta_{n}\right)$, $\mathrm{D}\left(\operatorname{Re}\left(z_{j, n}\right)\right)$ and $\mathrm{D}\left(\operatorname{Im}\left(z_{j, n}\right)\right)$, limited to sums of a few terms. Reaching an high order in the expansions is somehow lengthy but allows to control better the smallness of the tail

$$
\mathrm{tl}_{G_{n}}:=\mathrm{M}\left(G_{n}\right)-\Pi_{G_{n}}
$$

in the asymptotic expansion of the Mahler measure.

Table 1 (the page 92) reports the state of the art for the expressions "D" and "tl".

\section{The basic questions are the following:}

(i) Whether the family $\left(\Pi_{G_{n}}\right)_{n \geq 2}$ is such that there exists $b>1$ such that

$$
b<\Pi_{G_{n}} \text { for all } n \geq 2 \text {. }
$$

(ii) Whether $\liminf _{n \rightarrow+\infty} \Pi_{G_{n}}$ exists.

(iii) Whether the $\operatorname{limit}_{n \rightarrow+\infty} \operatorname{tl}\left(G_{n}\right)$ is equal to zero.

The expansions of the roots given by Proposition 3.1 and Proposition 3.4 allow to answer positively to these questions (in Section 4.2 and Section 5).

Expanding $\theta_{n}, n \geq 2$, by the method of Poincaré (in Proposition 3.1) leads to asymptotic expansions of $\theta_{n}$ as divergent formal series of functions of only one variable: which is " $n$ ", the degree of $G_{n}$. 


\section{JEAN-LOUIS VERGER-GAUGRY}

TABLE 1. Expressions of $\mathrm{D}\left(\theta_{n}\right), \mathrm{D}\left(\Re\left(z_{j, n}\right)\right)$ and $\mathrm{D}\left(\Im\left(z_{j, n}\right)\right)$ in Selmer [Sr] and Flatto, Lagarias and Poonen [FLP]. They are limited to first order terms, with focus on $\theta_{n}$ and the root $z_{1, n}$ the closest to $\theta_{n}$. The interest for the other roots $z_{j, n}, 2 \leq j \leq\lfloor n / 6\rfloor$, seems to have been negligible since no result has been found by the author.

\begin{tabular}{|c|c|c|}
\hline $\mathrm{D}$ & tail & \\
\hline $\mathrm{D}\left(\theta_{n}\right)=1-\frac{\log n}{n}$ & not given & {$[\mathrm{Sr}, \mathrm{p} 292]$} \\
$\mathrm{D}\left(\theta_{n}\right)=1-\frac{(\log n-\log \log n)}{n}$ & $\frac{1}{n} O\left(\frac{\log \log n}{\log n}\right)$ & {$[\mathrm{FLP}$, Lemma $5.1(\mathrm{i})]$} \\
\hline $\mathrm{D}\left(\Re\left(z_{1, n}\right)\right)=\theta_{n}+\frac{2 \pi^{2}}{n \log ^{2} n}$ & $O\left(\frac{\log \log n}{n \log ^{3} n}\right)$ & {$[\mathrm{FLP}$, Lemma $5.3(\mathrm{ii})]$} \\
$\mathrm{D}\left(\Im\left(z_{1, n}\right)\right)=\frac{2 \pi}{n}$ & $o\left(\frac{1}{n}\right)$ & {$[\mathrm{FLP}$, Lemma 5.3 (ii)] } \\
\hline
\end{tabular}

\section{Proposition 3.1.}

Let $n \geq 2$. The root $\theta_{n}$ can be expressed as: $\theta_{n}=\mathrm{D}\left(\theta_{n}\right)+\operatorname{tl}\left(\theta_{n}\right)$ with

$$
\begin{aligned}
\mathrm{D}\left(\theta_{n}\right)=1-\frac{\log n}{n} & \left(1-\left(\frac{n-\log n}{n \log n+n-\log n}\right)\right. \\
& \left.\times\left(\log \log n-n \log \left(1-\frac{\log n}{n}\right)-\log n\right)\right)
\end{aligned}
$$

and

$$
\operatorname{tl}\left(\theta_{n}\right)=\frac{1}{n} O\left(\left(\frac{\log \log n}{\log n}\right)^{2}\right) \text {, with the constant } 1 / 2 \text { involved in } O() \text {. }
$$

P r o of. Let us put $\theta_{n}=1-\frac{t}{n}$ with $0<t<n$. Then

$$
\frac{t}{n}=\left(1-\frac{t}{n}\right)^{n}
$$

Let us show that $t<\log n$. Let $g(x)=x e^{x}$ be the increasing function of the variable $x$ on $\mathbb{R}$. The equation (3.24) implies $\frac{t}{n}=\left(1-\frac{t}{n}\right)^{n}<e^{-t} \Leftrightarrow g(t)<n$. Since $n<n \log n$ for $n \geq 3$ and $g(\log n)=n \log n$, we deduce the claim.

Taking the logarithm of (3.24) we obtain

$$
\log t-\log n=n \log \left(1-\frac{t}{n}\right)=-t-\frac{1}{2} \frac{t^{2}}{n}-\frac{1}{3} \frac{t^{3}}{n^{2}}-\cdots
$$


The identity

$$
t+\log t+\frac{1}{2} \frac{t^{2}}{n}+\frac{1}{3} \frac{t^{3}}{n^{2}}+\cdots=\log n
$$

has now to be inversed in order to obtain $t$ as a function of $n$. For doing this, we put $t=\log n+w$. Equation (3.25) transforms into the following equation in $w$, $(\log n+w)+\log (\log n+w)+\frac{1}{2} \frac{(\log n+w)^{2}}{n}+\frac{1}{3} \frac{(\log n+w)^{3}}{n^{2}}+\cdots=\log n$.

We deduce

$$
\begin{aligned}
w+\log (\log n)+\log \left(1+\frac{w}{\log n}\right) & +\frac{1}{2} \frac{\log ^{2} n}{n}\left(1+\frac{w}{\log n}\right)^{2} \\
& +\frac{1}{3} \frac{\log ^{3}}{n^{2}}\left(1+\frac{w}{\log n}\right)^{3}+\cdots=0 .
\end{aligned}
$$

Since

$$
n \log \left(1-\frac{\log n}{n}\right)+\log n=-\frac{1}{2} \frac{\log ^{2} n}{n}-\frac{1}{3} \frac{\log ^{3} n}{n^{2}}-\cdots
$$

and that

$$
\log \left(1+\frac{w}{\log n}\right)=\frac{w}{\log n}-\frac{w^{2}}{2 \log ^{2} n}+\frac{w^{3}}{3 \log ^{3} n}-\cdots
$$

we have

$$
\begin{aligned}
\log \log n- & n \log \left(1-\frac{\log n}{n}\right)-\log n \\
=w & {\left[-1-\frac{1}{\log n}-\frac{\log n}{n}-\frac{\log ^{2} n}{n^{2}}-\frac{\log ^{3} n}{n^{3}}-\cdots\right] } \\
& +w^{2}\left[\frac{1}{2 \log ^{2} n}-\frac{1}{2 n}-\frac{\log n}{n^{2}}-\frac{6}{4} \frac{\log ^{2} n}{n^{3}}-\cdots\right]+\cdots
\end{aligned}
$$

The coefficient coeff $(w)$ of $w$ is

$$
-1-\frac{1}{\log n}-\frac{\log n}{n}\left(1+\frac{\log n}{n}+\left(\frac{\log n}{n}\right)^{2}+\cdots\right)=\frac{-n \log n-n+\log n}{(\log n)(n-\log n)} .
$$

We deduce

$$
w=\frac{(\log n)(n-\log n)\left(\log \log n-n \log \left(1-\frac{\log n}{n}\right)-\log n\right)}{-n \log n-n+\log n}+\cdots
$$

which gives the expression of $\mathrm{D}\left(\theta_{n}\right)$. 


\section{JEAN-LOUIS VERGER-GAUGRY}

Let us write $w$ in $(3.27)$ as $\mathrm{D}(w)+u$, where $u$ denotes the remainding terms. Putting $w=\mathrm{D}(w)+u$ in (3.26) we obtain, for large $n$,

$$
0=u \operatorname{coeff}(w)+\mathrm{D}(w)^{2} \frac{1}{2 \log ^{2} n}+\cdots
$$

Since, for large $n, \operatorname{coeff}(w) \cong-1$ and $\mathrm{D}(w) \cong-\log \log n$, we deduce

$$
u \cong O\left(\left(\frac{\log \log n}{\log n}\right)^{2}\right)
$$

with a constant $1 / 2$ involved in $O()$. We deduce the tail $\operatorname{tl}\left(\theta_{n}\right)$ of $\theta_{n}$.

LEMmA 3.2. Given the limited expansion $D\left(\theta_{n}\right)$ of $\theta_{n}$ as in (3.22), denote

$$
\lambda_{n}:=1-\left(1-\mathrm{D}\left(\theta_{n}\right)\right) \frac{n}{\log n} .
$$

Then

$$
\lambda_{n}=\mathrm{D}\left(\lambda_{n}\right)+\mathrm{tl}\left(\lambda_{n}\right)
$$

with

$$
\mathrm{D}\left(\lambda_{n}\right)=\frac{\log \log n}{\log n}\left(\frac{1}{1+\frac{1}{\log n}}\right), \quad \operatorname{tl}\left(\lambda_{n}\right)=O\left(\frac{\log \log n}{n}\right)
$$

with the constant 1 in the Big $O$.

P r o o f. Indeed, the identity $\mathrm{D}\left(\theta_{n}\right)=1-\frac{\log n}{n}\left(1-\lambda_{n}\right)$, from (3.22), readily gives the result.

In the sequel, for short, we write $\lambda_{n}$ instead of $\mathrm{D}\left(\lambda_{n}\right)$, which amounts to the same in the limited asymptotic expansions.

REMARK 3.3. Angular sectorization of the roots $z_{j, n}$, transition regions and notations used in Proposition 3.4.

For the roots $z_{j, n}$ lying in the first quadrant, the Poincaré asymptotic expansions of $z_{j, n}$ will be obtained below as divergent formal series of functions of the couple of two variables which is:

- $\left(n, \frac{j}{n}\right), \quad$ in the angular sector: $\frac{\pi}{2}>\arg z>2 \pi \frac{\log n}{n}$,

- $\left(n, \frac{j}{\log n}\right)$, in the angular sector ("bump" sector)

$$
2 \pi \frac{\log n}{n}>\arg z \geq 0 \text {. }
$$


Moreover expanding the roots at the fourth order has for consequence to dispatch the roots $z_{j, n}$ lying in the bump sector into the two subsectors:

- $2 \pi \frac{\sqrt{(\log n)(\log \log n)}}{n}>\arg z>0$,

- $2 \pi \frac{\log n}{n}>\arg z>2 \pi \frac{\sqrt{(\log n)(\log \log n)}}{n}$.

As compared with the (full) sector $\frac{\pi}{3}>\arg z>0$, the relative size of the bump sector is negligible, as soon as $n$ is large enough. By transition region, we mean a small neighbourhood of the argument

$$
\arg z=2 \pi \frac{\log n}{n}, \quad \text { or of } \quad 2 \pi \frac{\sqrt{(\log n)(\log \log n)}}{n} .
$$

Outside these two transition regions, one unique dominant asymptotic expansion of $z_{j, n}$ exists. In a transition region an asymptotic expansion contains more second- or third- or fourth- order terms, of the same order of magnitude, which should be taken into account together. Let us define more precisely these neighbourhoods. Let $\epsilon \in(0,1)$. We introduce two strictly increasing sequences of real numbers $\left(u_{n}\right),\left(v_{n}\right)$ which satisfy: for $n \geq n_{0}=18$,

$$
\lfloor n / 6\rfloor>v_{n}>\log n, \quad \log n>u_{n}>\sqrt{(\log n)(\log \log n)},
$$

such that

$$
\lim _{n \rightarrow \infty} \frac{v_{n}}{n}=\lim _{n \rightarrow \infty} \frac{\sqrt{(\log n)(\log \log n)}}{u_{n}}=\lim _{n \rightarrow \infty} \frac{u_{n}}{\log n}=\lim _{n \rightarrow \infty} \frac{\log n}{v_{n}}=0
$$

and

$$
v_{n}-u_{n}=O\left((\log n t)^{1+\epsilon}\right)
$$

with the constant 1 involved in the big $\mathrm{O}$.

The roots $z_{j, n}$ lying in the first transition region about $2 \pi(\log n) / n$ are such that

$$
2 \pi \frac{v_{n}}{n}>\arg z_{j, n}>2 \pi \frac{\left(2 \log n-v_{n}\right)}{n} .
$$

The roots $z_{j, n}$ lying in the second transition region about

$$
2 \pi \sqrt{(\log n)(\log \log n)} / n
$$

are such that

$$
2 \pi \frac{u_{n}}{n}>\arg z_{j, n}>2 \pi \frac{2 \sqrt{(\log n)(\log \log n)}-u_{n}}{n} .
$$

In Proposition 3.4, for simplicity's sake, these two transition regions are schematically denoted by

$$
\arg z \asymp 2 \pi \frac{(\log n)}{n}, \quad \text { respectively, } \quad \arg z \asymp 2 \pi \frac{\sqrt{(\log n)(\log \log n)}}{n} .
$$


By complementarity, the other sectors are schematically written:

instead of

$$
2 \pi \frac{\sqrt{(\log n)(\log \log n)}}{n}>\arg z>0
$$

$$
2 \pi \frac{2 \sqrt{(\log n)(\log \log n)}-u_{n}}{n}>\arg z>0,
$$

respectively,

$$
2 \pi \frac{\log n}{n}>\arg z 2 \pi \frac{\sqrt{(\log n)(\log \log n)}}{n}
$$

instead of

$$
2 \pi \frac{2 \log n-v_{n}}{n}>\arg z>2 \pi \frac{u_{n}}{n},
$$

respectively,

$$
\frac{\pi}{2}>\arg z>2 \pi \frac{\log n}{n} \quad \text { instead of } \frac{\pi}{2}>\arg z>2 \pi \frac{v_{n}}{n} .
$$

The asymptotic expansions of the roots $z_{j, n}$ lying in the transition region $\arg z \asymp 2 \pi \frac{\log n}{n}$ are not reported in Proposition 3.4; they are left to the reader, and can be easily obtained by superimposition of those of the two cases (i) and (ii) in Proposition 3.4.

In the following asymptotic expansions, the values of $n$ which will be considered are greater than a critical value $n_{0}$ for which $2 \pi \frac{\log n}{n}$, is small enough compared to $\pi / 3$ for all $n \geq n_{0}$. Arbitrarily we take $n_{0}=18$ in the sequel and in Theorem 1.2 (cf. Figure 1, on the page 88 to visualize the geometry of the roots of $\left.G_{51}^{*}\right)$.

Proposition 3.4. Let $n \geq n_{0}=18$ and $1 \leq j \leq\left\lfloor\frac{n-1}{4}\right\rfloor$. The roots $z_{j, n}$ of $G_{n}(X)$ have the following asymptotic expansions, $z_{j, n}=\mathrm{D}\left(z_{j, n}\right)+\operatorname{tl}\left(z_{j, n}\right)$, in the following angular sectors:

(i) "Bump" sector $2 \pi \frac{\log n}{n}>\arg z>0$ :

- Subsector $2 \pi \frac{\sqrt{(\log n)(\log \log n)}}{n}>\arg z>0$ :

$$
\begin{aligned}
& \mathrm{D}\left(\Re\left(z_{j, n}\right)\right)=\theta_{n}+\frac{2 \pi^{2}}{n}\left(\frac{j}{\log n}\right)^{2}\left(1+2 \lambda_{n}\right), \\
& \mathrm{D}\left(\Im\left(z_{j, n}\right)\right)=\frac{2 \pi \log n}{n}\left(\frac{j}{\log n}\right)\left[1-\frac{1}{\log n}\left(1+\lambda_{n}\right)\right],
\end{aligned}
$$

with

$$
\operatorname{tl}\left(\Re\left(z_{j, n}\right)\right)=\frac{1}{n \log n}\left(\frac{j}{\log n}\right)^{2} O\left(\left(\frac{\log \log n}{\log n}\right)^{2}\right),
$$




$$
\operatorname{tl}\left(\Im\left(z_{j, n}\right)\right)=\frac{1}{n \log n}\left(\frac{j}{\log n}\right) O\left(\left(\frac{\log \log n}{\log n}\right)^{2}\right),
$$

- Subsector $2 \pi \frac{\log n}{n}>\arg z>2 \pi \frac{\sqrt{(\log n)(\log \log n)}}{n}$ :

$$
\begin{aligned}
\mathrm{D}\left(\Re\left(z_{j, n}\right)\right)= & \theta_{n}+\frac{2 \pi^{2}}{n}\left(\frac{j}{\log n}\right)^{2}\left(1+\frac{2 \pi^{2}}{3}\left(\frac{j}{\log n}\right)^{2}\left(1+\lambda_{n}\right)\right) \\
\mathrm{D}\left(\Im\left(z_{j, n}\right)\right)= & \frac{2 \pi \log n}{n}\left(\frac{j}{\log n}\right) \\
& \times\left[1-\frac{1}{\log n}\left(1-\frac{4 \pi^{2}}{3}\left(\frac{j}{\log n}\right)^{2}\left(1-\frac{1}{\log n}\left(1-\lambda_{n}\right)\right)\right)\right],
\end{aligned}
$$

with

$$
\operatorname{tl}\left(\Re\left(z_{j, n}\right)\right)=\frac{1}{n} O\left(\left(\frac{j}{\log n}\right)^{6}\right), \quad \operatorname{tl}\left(\Im\left(z_{j, n}\right)\right)=\frac{1}{n} O\left(\left(\frac{j}{\log n}\right)^{5}\right),
$$

- Transition region $\arg z \asymp 2 \pi \frac{\sqrt{(\log n)(\log \log n)}}{n}$ :

$$
\begin{aligned}
\mathrm{D}\left(\Re\left(z_{j, n}\right)\right)= & \theta_{n}+\frac{2 \pi^{2}}{n}\left(\frac{j}{\log n}\right)^{2}\left(1+2 \lambda_{n}+\frac{2 \pi^{2}}{3}\left(\frac{j}{\log n}\right)^{2}\left(1+\lambda_{n}\right)\right), \\
\mathrm{D}\left(\Im\left(z_{j, n}\right)\right)= & \frac{2 \pi \log n}{n}\left(\frac{j}{\log n}\right) \times\left[1-\frac{1}{\log n}\right. \\
& \left.\times\left(1+\lambda_{n}-\frac{4 \pi^{2}}{3}\left(\frac{j}{\log n}\right)^{2}\left(1-\frac{1}{\log n}\left(1-\lambda_{n}\right)\right)\right)\right],
\end{aligned}
$$

with

$$
\operatorname{tl}\left(\Re\left(z_{j, n}\right)\right)=\frac{1}{n} O\left(\left(\frac{\log \log n}{\log n}\right)^{3}\right), \operatorname{tl}\left(\Im\left(z_{j, n}\right)\right)=\frac{1}{n} O\left(\left(\frac{\log \log n}{\log n}\right)^{5 / 2}\right),
$$

(ii) Sector $\frac{\pi}{2}>\arg z>2 \pi \frac{\log n}{n}$ :

$$
\begin{aligned}
& \mathrm{D}\left(\Re\left(z_{j, n}\right)\right)=\cos \left(2 \pi \frac{j}{n}\right)+\frac{\log \left(2 \sin \left(\pi \frac{j}{n}\right)\right)}{n}, \\
& \mathrm{D}\left(\Im\left(z_{j, n}\right)\right)=\sin \left(2 \pi \frac{j}{n}\right)+\tan \left(\pi \frac{j}{n}\right) \frac{\log \left(2 \sin \left(\pi \frac{j}{n}\right)\right)}{n}
\end{aligned}
$$

with

$$
\operatorname{tl}\left(\Re\left(z_{j, n}\right)\right)=\operatorname{tl}\left(\Im\left(z_{j, n}\right)\right)=\frac{1}{n} O\left(\left(\frac{\log \log n}{\log n}\right)^{2}\right) .
$$




\section{JEAN-LOUIS VERGER-GAUGRY}

Proof. Let us write the expression of the $j$ th-root $z_{j, n}$ as

$$
z_{j, n}=\theta_{n} e^{2 i \pi\left(\frac{j}{n}+\phi_{j, n}\right)}
$$

where $\phi_{j, n}$ a complex number which is assumed to be of small modulus. This assumption on $\phi_{j, n}$ will be confirmed in each case. Let us denote, for short, by $\Re$ the real part of $\phi_{j, n}$, resp. by $\Im$, the imaginary part of $\phi_{j, n}$. The determination of $\phi_{j, n}$ will be done in the sequel by giving expressions of $\mathrm{D}(\Re)$ and $\mathrm{D}(\Im)$ and of their corresponding tails $\operatorname{tl}(\Re)$ and $\operatorname{tl}(\Im)$.

Since $\theta_{n}^{n}=1-\theta_{n}$, the equation $G_{n}\left(z_{j, n}\right)=0$ implies

Then

$$
0=1-\theta_{n} e^{2 i \pi\left(\frac{j}{n}+\Re+i \Im\right)}-\left(1-\theta_{n}\right) e^{2 i n \pi(\Re+i \Im)} .
$$

$$
0=1-\theta_{n} \cos \left(2 \pi\left(\frac{j}{n}+\Re\right)\right) e^{-2 \pi \Im}-\left(1-\theta_{n}\right) \cos (2 \pi n \Re) e^{-2 \pi n \Im},
$$

and

$$
0=-\theta_{n} \sin \left(2 \pi\left(\frac{j}{n}+\Re\right)\right) e^{-2 \pi \Im}-\left(1-\theta_{n}\right) \sin (2 \pi n \Re) e^{-2 \pi n \Im} .
$$

We will assume throughout the rest of the proof that $\Re=o(1)$ and $\Im=o(1)$ when $n$ tends to infinity, whatever the value of $j$ in $\left\{1,2, \ldots,\left\lfloor\frac{n-1}{2}\right\rfloor\right\}$; hence $\sin (2 \pi \Re) \cong 2 \pi \Re$ and $\cos (2 \pi \Re) \cong 1-2 \pi^{2} \Re^{2}$ for large $n$. Then (3.32) implies

$$
\frac{\theta_{n}}{1-\theta_{n}}\left(\sin \left(\frac{2 \pi j}{n}\right)\left(1-2 \pi^{2} \Re^{2}\right)+2 \pi \Re \cos \left(\frac{2 \pi j}{n}\right)\right)=-\sin (2 \pi n \Re) e^{-2 \pi(n-1) \Im} .
$$

“Bump" Sector $2 \pi \frac{\log n}{n}>\arg z>0$ :

In this case $j / \log n=o(1)$, for all $j$ with $z_{j, n}$ in this sector, and then $n \Re=o(1)$ and $n \Im=o(1)$. In fact, we will consider these two last conditions as assumptions in making the expansions and will show that they are checked at the end. For large $n, \sin (2 \pi n \Re) \cong 2 \pi n \Re-\frac{4 \pi^{3}}{3} n^{3} \Re^{3}$ and $e^{-2 \pi(n-1) \Im} \cong$ $1-2 \pi(n-1) \Im+2 \pi^{2}(n-1)^{2} \Im^{2}$. From (3.33) we deduce, up the third-order terms,

$$
\begin{aligned}
& \frac{\theta_{n}}{2 \pi\left(1-\theta_{n}\right)} \sin \left(\frac{2 \pi j}{n}\right)=\Re\left[-\frac{\theta_{n}}{1-\theta_{n}} \cos \left(\frac{2 \pi j}{n}\right)-n\right]+\Re^{2}\left[\pi \frac{\theta_{n}}{1-\theta_{n}} \sin \left(\frac{2 \pi j}{n}\right)\right] \\
& +\Re \Im[2 \pi n(n-1)]+\Re \Im^{2}\left[2 \pi^{2} n(n-1)^{2}\right]+\Re^{3}\left[\frac{2 \pi^{2}}{3} \frac{\theta_{n}}{1-\theta_{n}} \cos \left(\frac{2 \pi j}{n}\right)+\frac{2 \pi^{2}}{3} n^{3}\right]+\cdots
\end{aligned}
$$


In the rhs of (3.34) only the first term is the dominant one. Then

$$
\Re=-\frac{\sin \left(\frac{2 \pi j}{n}\right)}{2 \pi\left(\cos \left(\frac{2 \pi j}{n}\right)+n \frac{1-\theta_{n}}{\theta_{n}}\right)}+u,
$$

where $u$ represents the remaining terms. For large $n$, since $\sin \left(\frac{2 \pi j}{n}\right) \cong \frac{2 \pi j}{n}$ and $\frac{\mathrm{D}\left(\theta_{n}\right)}{n\left(1-\mathrm{D}\left(\theta_{n}\right)\right)} \cong \frac{1}{\log n}$, using (3.22), we obtain the following approximant expression

$$
\mathrm{D}\left(-\frac{\sin \left(\frac{2 \pi j}{n}\right)}{2 \pi\left(\cos \left(\frac{2 \pi j}{n}\right)+n \frac{1-\theta_{n}}{\theta_{n}}\right)}\right)=\mathrm{D}(\Re)=\frac{-j}{n \log n},
$$

with

$$
\begin{aligned}
\mathrm{tl}\left(-\frac{\sin \left(\frac{2 \pi j}{n}\right)}{2 \pi\left(\cos \left(\frac{2 \pi j}{n}\right)+n \frac{1-\theta_{n}}{\theta_{n}}\right)}\right)= & \frac{-j}{n \log n} \lambda_{n} \\
& +\frac{j}{n \log ^{2} n} O\left(\left(\frac{\log \log n}{\log n}\right)^{2}\right) .
\end{aligned}
$$

From (3.36) we readily deduce that the approximation conditions $\Re=o(1)$ and $n \Re=o(1)$ we have taken are valid.

Let us now turn to the imaginary part of $\phi_{j, n}$. Multiplying (3.31) by $e^{2 \pi \Im}$, with the same assumptions as above: $n \Re=o(1)$ and $n \Im=o(1)$, we obtain

$$
\begin{aligned}
0= & \left(1+2 \pi \Im+2 \pi^{2} \Im^{2}+\frac{4 \pi^{3}}{3} \Im^{3}\right) \\
& -\theta_{n}\left[\cos \left(\frac{2 \pi j}{n}\right)\left(1-2 \pi^{2} \Re^{2}+\left(2 \pi^{4} / 3\right) \Re^{4}\right)-\sin \left(\frac{2 \pi j}{n}\right)\left(2 \pi \Re-\frac{4 \pi^{3}}{3} \Re^{3}\right)\right] \\
& -\left(1-\theta_{n}\right)\left(1-2 \pi^{2} n^{2} \Re^{2}+\frac{2 \pi^{4}}{3} n^{4} \Re^{4}\right) \\
& \times\left(1-2 \pi(n-1) \Im+2 \pi^{2}(n-1)^{2} \Im^{2}-\frac{4 \pi^{3}}{3}(n-1)^{3} \Im^{3}\right)+\cdots
\end{aligned}
$$

Then

$$
\begin{aligned}
0= & -\theta_{n}\left(\cos \left(\frac{2 \pi j}{n}\right)-1\right)+\Re\left[2 \pi \theta_{n} \sin \left(\frac{2 \pi j}{n}\right)\right] \\
& +\Re^{2}\left[2 \pi^{2} \theta_{n} \cos \left(\frac{2 \pi j}{n}\right)+2 \pi^{2} n^{2}\left(1-\theta_{n}\right)\right] \\
& +\Im\left[2 \pi+2 \pi(n-1)\left(1-\theta_{n}\right)\right]+\Im^{2}\left[2 \pi^{2}-2 \pi^{2}(n-1)^{2}\left(1-\theta_{n}\right)\right]
\end{aligned}
$$




$$
\begin{aligned}
& +\Re^{3}\left[-\frac{4 \pi^{3}}{3} \theta_{n} \sin \left(\frac{2 \pi j}{n}\right)\right]+\Re^{2} \Im\left[-4 \pi^{3}\left(1-\theta_{n}\right) n^{2}(n-1)\right] \\
& +\Im^{3}\left[\frac{4 \pi^{3}}{3}+\frac{4 \pi^{3}}{3}\left(1-\theta_{n}\right)(n-1)^{3}\right] \\
& +\Re^{4}\left[-\frac{2 \pi^{4}}{3} \theta_{n} \cos \left(\frac{2 \pi j}{n}\right)-\frac{2 \pi^{4}}{3} n^{4}\left(1-\theta_{n}\right)\right]+\cdots
\end{aligned}
$$

Among the first four terms in the rhs in (3.39), the 3rd and 4th terms are the dominant ones for large $n$. Except these two dominant terms, all the other terms of order $\geq 2$ are negligible for a first-order approximation. Then, replacing $\Re$ as obtained in (3.35), we deduce

$$
\Im=-\frac{1}{4 \pi} \frac{\left(\sin ^{2}\left(\frac{2 \pi j}{n}\right)\right)\left(\cos \left(\frac{2 \pi j}{n}\right)+n^{2} \frac{1-\theta_{n}}{\theta_{n}}\right)}{\left(\cos \left(\frac{2 \pi j}{n}\right)+n \frac{1-\theta_{n}}{\theta_{n}}\right)^{2}\left(1+n \frac{1-\theta_{n}}{\theta_{n}}\right)}+v,
$$

where $v$ represents the remaining terms. For large $n$, using (3.22), we obtain the following approximant expression

$$
\mathrm{D}\left(-\frac{1}{4 \pi} \frac{\left(\sin ^{2}\left(\frac{2 \pi j}{n}\right)\right)\left(\cos \left(\frac{2 \pi j}{n}\right)+n^{2} \frac{1-\theta_{n}}{\theta_{n}}\right)}{\left(\cos \left(\frac{2 \pi j}{n}\right)+n \frac{1-\theta_{n}}{\theta_{n}}\right)^{2}\left(1+n \frac{1-\theta_{n}}{\theta_{n}}\right)}\right)=\mathrm{D}(\Im)=\frac{-\pi j^{2}}{n \log ^{2} n},
$$

with

$$
\begin{gathered}
\mathrm{tl}\left(-\frac{1}{4 \pi} \frac{\left(\sin ^{2}\left(\frac{2 \pi j}{n}\right)\right)\left(\cos \left(\frac{2 \pi j}{n}\right)+n^{2} \frac{1-\theta_{n}}{\theta_{n}}\right)}{\left(\cos \left(\frac{2 \pi j}{n}\right)+n \frac{1-\theta_{n}}{\theta_{n}}\right)^{2}\left(1+n \frac{1-\theta_{n}}{\theta_{n}}\right)}\right) \\
=\frac{-2 \pi j^{2}}{n \log ^{2} n} \lambda_{n}+\frac{j^{2}}{n \log ^{3} n} O\left(\left(\frac{\log \log n}{\log n}\right)^{2}\right) .
\end{gathered}
$$

The expression (3.41) implies that the approximation conditions $\Im=o(1)$ and $n \Im=o(1)$ are valid, and (3.36) together with (3.41) both imply the negligibility of above-mentioned higher-order terms in (3.34) and (3.39). Thus the approximations made are a posteriori justified. Let us now improve the limited expansions $\mathrm{D}(\Re)$ and $\mathrm{D}(\Im)$ given by (3.36) and (3.41) with higher-order terms, namely with some (limited) expansions $\mathrm{D}(u)$ and $\mathrm{D}(v)$ of $u$ and $v$.

In the rhs of (3.34), except the first one, the 3rd term and the 5th term are the dominant terms among all the others. Replacing the expression (3.35) of $\Re$ in (3.34) gives

$$
0=u\left(-\frac{\theta_{n}}{1-\theta_{n}} \cos \left(\frac{2 \pi j}{n}\right)-n\right)+\frac{4 \pi^{2}}{3} \frac{j^{3}}{\log ^{3} n}+O\left(\frac{j^{5}}{\log ^{5} n}\right) .
$$


Therefore,

$$
u=\frac{4 \pi^{2}}{3} \frac{j^{3}}{n \log ^{3} n}\left(1-\frac{n}{\log ^{2} n}\left(1-\mathrm{D}\left(\theta_{n}\right)\right)\right)+\frac{1}{n} O\left(\left(\frac{j}{\log n}\right)^{5}\right) .
$$

Denote $\mathrm{D}\left(\mathrm{tl}_{\Re}\right):=\frac{-j}{n \log n} \lambda_{n}$ the first term in the rhs of (3.37) and

$$
\mathrm{D}(u):=\frac{4 \pi^{2}}{3} \frac{j^{3}}{n \log ^{3} n}\left(1-\frac{1}{\log n}\left(1-\lambda_{n}\right)\right) .
$$

According to the range of values taken by $j$, the expressions $\mathrm{D}(u)$ and $\mathrm{D}\left(\mathrm{tl}_{\Re}\right)$ for large $n$ are either of similar orders of magnitude, or one is completely dominant over the other. The comparison between both is easy and, with $\mathrm{D}\left(\theta_{n}\right)$ given by (3.22), readily implies the following expression of $\mathrm{D}(\Re)$.

(i) Subsector $2 \pi \frac{\sqrt{(\log n)(\log \log n)}}{n}>\arg z>0$ :

in this case, $j / \sqrt{(\log n)(\log \log n)}=o(1)$ for all $j$ such that $z_{j, n}$ belongs to this subsector; $\mathrm{D}\left(\mathrm{tl}_{\Re}\right)$ is dominant over $\mathrm{D}(u)$ and the new (limited) expansion of $\Re=\Re\left(\phi_{j, n}\right)$ is

with

$$
\mathrm{D}(\Re)=-\frac{j}{n \log n}\left(1+\lambda_{n}\right)
$$

$$
\operatorname{tl}(\Re)=\frac{j}{n \log ^{2} n} O\left(\left(\frac{\log \log n}{\log n}\right)^{2}\right) .
$$

(ii) Subsector $2 \pi \frac{\log n}{n}>\arg z>2 \pi \frac{\sqrt{(\log n)(\log \log n)}}{n}$ :

in this case, $(\sqrt{(\log n)(\log \log n)}) / j=o(1)$, for all $j$ such that $z_{j, n}$ belongs to this subsector; $\mathrm{D}(u)$ is dominant over $\mathrm{D}\left(\mathrm{tl}_{\Re}\right)$, and then

$$
\mathrm{D}(\Re)=-\frac{j}{n \log n}\left(1-\frac{4 \pi^{2}}{3} \frac{j^{2}}{\log ^{2} n}\left(1-\frac{1}{\log n}\left(1-\lambda_{n}\right)\right)\right)
$$

with

$$
\operatorname{tl}(\Re)=\frac{1}{n} O\left(\left(\frac{j}{\log n}\right)^{5}\right) .
$$

(iii) Transition region $j \asymp \sqrt{(\log n)(\log \log n)}$ :

both expansions have to be considered and

$$
\mathrm{D}(\Re)=\frac{-j}{n \log n}\left(1+\lambda_{n}-\frac{4 \pi^{2}}{3} \frac{j^{2}}{\log ^{2} n}\left(1-\frac{1}{\log n}\left(1-\lambda_{n}\right)\right)\right)
$$


with

$$
\operatorname{tl}(\Re)=\frac{1}{n} O\left(\left(\frac{\log \log n}{\log n}\right)^{5 / 2}\right) .
$$

In the rhs of (3.39) the 5th, 7 th and 9 th terms are the dominant remaining terms among all the others. Replacing the expressions (3.36) of $\Re$ and (3.41) of $\Im$ in $(3.39)$ gives

$$
0=2 \pi v\left[1+(n-1)\left(1-\theta_{n}\right)\right]+\frac{j^{4}}{n \log ^{3} n}\left[-2 \pi^{4}+4 \pi^{4}-\frac{2 \pi^{4}}{3}\right]+\cdots
$$

Then

$$
v=\frac{-2 \pi^{3}}{3} \frac{j^{4}}{n \log ^{4} n}\left(2-\left(1-\mathrm{D}\left(\theta_{n}\right)\right) \frac{n}{\log n}\right)+\frac{1}{n} O\left(\left(\frac{j}{\log n}\right)^{6}\right) .
$$

Denote $\mathrm{D}\left(\mathrm{tl}_{\Im}\right):=\frac{-2 \pi j^{2}}{n \log ^{2} n} \lambda_{n}$ the first term in the rhs of $(3.42)$ and

$$
\mathrm{D}(v):=\frac{-2 \pi^{3}}{4} \frac{j^{4}}{n \log ^{4} n}\left(1+\lambda_{n}\right) .
$$

We now compare the expressions $\mathrm{D}(v)$ and $\mathrm{D}\left(\mathrm{tl}_{\Im}\right)$ for large $n$ to check which one is dominant over the other. It readily implies the following expression of $\mathrm{D}(\Im)$.

(i) Subsector $2 \pi \frac{\sqrt{(\log n)(\log \log n)}}{n}>\arg z>0$ :

$\mathrm{D}\left(\mathrm{tl}_{\Im}\right)$ is dominant over $\mathrm{D}(v)$ and the new (limited) expansion of $\Im=$ $\Im\left(\phi_{j, n}\right)$ is

$$
\mathrm{D}(\Im)=\frac{-\pi j^{2}}{n \log ^{2} n}\left(1+2 \lambda_{n}\right)
$$

with

$$
\operatorname{tl}(\Im)=\frac{j^{2}}{n \log ^{3} n} O\left(\left(\frac{\log \log n}{\log n}\right)^{2}\right)
$$

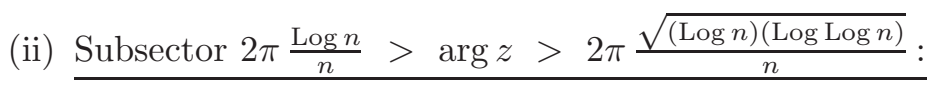

$\mathrm{D}(v)$ is dominant over $\mathrm{D}\left(\mathrm{tl}_{\Im}\right)$, and then

with

$$
\mathrm{D}(\Im)=-\frac{\pi j^{2}}{n \log ^{2} n}\left(1+\frac{2 \pi^{2}}{3} \frac{j^{2}}{\log ^{2} n}\left(1+\lambda_{n}\right)\right)
$$

$$
\operatorname{tl}(\Im)=\frac{1}{n} O\left(\left(\frac{j}{\log n}\right)^{6}\right) \text {. }
$$


(iii) Transition region: $j \asymp \sqrt{(\log n)(\log \log n)}$ :

both expansions have to be considered and

with

$$
\mathrm{D}(\Im)=-\frac{\pi j^{2}}{n \log ^{2} n}\left[1+2 \lambda_{n}+\frac{2 \pi^{2}}{3} \frac{j^{2}}{\log ^{2} n}\left(1+\lambda_{n}\right)\right]
$$

$$
\operatorname{tl}(\Im)=\frac{1}{n} O\left(\left(\frac{\log \log n}{\log n}\right)^{3}\right) .
$$

Sector $\frac{\pi}{2}>\arg z>2 \pi \frac{\log n}{n}:$

In this case, $(\log n) / j=o(1)$ for all $j$ such that $z_{j, n}$ belongs to this sector. Taking the squares of (3.31) and of (3.32) and summing them up lead to the removal of the terms $\sin (2 \pi n \Re)$ and $\cos (2 \pi n \Re)$. We obtain

$$
\begin{aligned}
\left(\theta_{n}-1\right)^{2} e^{-4 \pi n \Im} & =\theta_{n}^{2} e^{-4 \pi \Im} \\
& -2 \theta_{n} e^{-2 \pi \Im}\left[\cos \left(\frac{2 \pi j}{n}\right) \cos (2 \pi \Re)-\sin \left(\frac{2 \pi j}{n}\right) \sin (2 \pi \Re)\right]+1 .
\end{aligned}
$$

Then, with the assumptions $\Re=o(1)$ and $\Im=o(1)$, (3.50) becomes

$$
\begin{aligned}
e^{-4 \pi n \Im}=1+\frac{2 \theta_{n}}{\left(\theta_{n}-1\right)^{2}}[ & -2 \pi \Im\left(\theta_{n}-\cos \left(\frac{2 \pi j}{n}\right)\right) \\
& \left.+2 \pi \Re \sin \left(\frac{2 \pi j}{n}\right)+\left(1-\cos \left(\frac{2 \pi j}{n}\right)\right)\right]+\cdots
\end{aligned}
$$

Let us observe that $\lim _{n \rightarrow+\infty} \theta_{n} /\left(\theta_{n}-1\right)^{2}=+\infty$, and that the term within brackets is positive and less than (say) 3 in modulus. Then, by taking the logarithm of (3.51), we deduce

$$
\begin{aligned}
\Im= & \frac{-1}{4 \pi n}\left[\log \left(\frac{\theta_{n}}{\left(\theta_{n}-1\right)^{2}}\right)+\log \left(2\left(1-\cos \left(\frac{2 \pi j}{n}\right)\right)\right)\right. \\
& \left.+\log \left(1-2 \pi \Im\left(\frac{\theta_{n}-\cos \left(\frac{2 \pi j}{n}\right)}{1-\cos \left(\frac{2 \pi j}{n}\right)}\right)+2 \pi \Re\left(\frac{\sin \left(\frac{2 \pi j}{n}\right)}{1-\cos \left(\frac{2 \pi j}{n}\right)}\right)\right)\right]+\cdots
\end{aligned}
$$

Let us give a first-order expansion of $\mathrm{D}(\Im)$ from (3.52) under the assumption that the quantities $2 \pi \Im\left(\frac{\theta_{n}-\cos \left(\frac{2 \pi j}{n}\right)}{1-\cos \left(\frac{2 \pi j}{n}\right)}\right)$ are $=o(1)$ for all $j$. This assumption 
will imply that the quantities $2 \pi \Re\left(\frac{\sin \left(\frac{2 \pi j}{n}\right)}{1-\cos \left(\frac{2 \pi j}{n}\right)}\right)$ also have the same property. We will check below that these assumptions are valid. First, by (3.28), we have

$$
\begin{gathered}
\log \left(\frac{\theta_{n}}{\left(\theta_{n}-1\right)^{2}}\right)=\log \theta_{n}-2 \log \left(1-\theta_{n}\right) \\
=-\frac{\log n}{n}\left(1-\lambda_{n}\right)-2 \log \left(\frac{\log n}{n}\left(1-\lambda_{n}\right)\right)+\cdots \\
=(2 \log n)\left[1-\frac{\log \log n}{\log n}+\frac{\lambda_{n}}{\log n}\right]+\lambda_{n}^{2}+\frac{2}{3} \lambda_{n}^{3}+\cdots \\
=(2 \log n)\left[1-\frac{\log \log n}{\log n}+\frac{\log \log n}{(\log n)(1+\log n)}\right]+O\left(\left(\frac{\log \log n}{\log n}\right)^{2}\right) \\
=(2 \log n)\left[1-\lambda_{n}\right]+O\left(\left(\frac{\log \log n}{\log n}\right)^{2}\right)
\end{gathered}
$$

with the constant 1 involved in the Big O. Expanding the last Logarithm at the first-order in (3.52) implies that the solution of (3.52) is $\Im=\mathrm{D}(\Im)+\operatorname{tl}(\Im)$ with

and

$$
\mathrm{D}(\Im)=\frac{-1}{2 \pi n}\left[(\log n)\left(1-\lambda_{n}\right)+\log \left(2 \sin \left(\frac{\pi j}{n}\right)\right)\right]
$$

$$
\operatorname{tl}(\Im)=+\frac{1}{n} O\left(\left(\frac{\log \log n}{\log n}\right)^{2}\right),
$$

such that the constant involved in the Big $\mathrm{O}$ in (3.56) is equal to $1 / 4 \pi$ (and does not depend upon $j$ ). Let us show that the assumption about

$$
\begin{aligned}
& -2 \pi \mathrm{D}(\Im)\left(\frac{\theta_{n}-\cos \left(\frac{2 \pi j}{n}\right)}{1-\cos \left(\frac{2 \pi j}{n}\right)}\right) \\
& =\frac{1}{n}\left[(\log n)\left(1-\mathrm{D}\left(\lambda_{n}\right)\right)+\log \left(2 \sin \left(\frac{\pi j}{n}\right)\right)\right]\left(\frac{\theta_{n}-1}{1-\cos \left(\frac{2 \pi j}{n}\right)}+1\right)
\end{aligned}
$$

is valid. Indeed, the function $F(x):=(\log (2 \sin x)) /(1-\cos (2 x))$ takes negative values on $[\pi(\log n) / n, \pi / 6]$ with positive derivative on this interval. Then checking at $x=\pi(\log n) / n$ is sufficient to obtain an upper bound of the quantity (3.57) in modulus. Taking for $\pi j / n$ the quantity $\pi(\log n) / n$, assuming $n$ large enough, it is easy to check that (3.57) is equivalent to

$$
\cong-\frac{1}{2 \pi^{2}} \frac{\log \log n}{(\log n)^{2}}
$$

uniformly in $j$, for all the roots $z_{j, n}$ in this sector. 
By truncating the power series $\log \left(1-\lambda_{n}\right)=-\lambda_{n}-\frac{1}{2} \lambda_{n}^{2}-\frac{1}{3} \lambda_{n}^{3}-\cdots$ in (3.53) into the sum of a polynomial section in $\lambda_{n}$ and a remainder, let us observe that, whatever the degree of this polynomial section is, the resulting tail in (3.54) is always the same, as a function of $n$, and the term $\log \theta_{n}$ in (3.52) asymptotically negligible. This observation allows to restore (3.52), using only the dominant terms and (3.53) with the full series, as

$\Im=\frac{-1}{4 \pi n}\left[\log \left(\frac{1}{\left(\theta_{n}-1\right)^{2}}\right)+2 \log \left(2 \sin \left(\frac{\pi j}{n}\right)\right)\right]+\frac{1}{n} O\left(\left(\frac{\log \log n}{\log n}\right)^{2}\right)$.

From (3.59) and from the expansion of the term within brackets [ ] in (3.52), we deduce that the following summation

$$
\log \theta_{n}-2 \pi \Im\left(\frac{\theta_{n}-\cos \left(\frac{2 \pi j}{n}\right)}{1-\cos \left(\frac{2 \pi j}{n}\right)}\right)+2 \pi \Re\left(\frac{\sin \left(\frac{2 \pi j}{n}\right)}{1-\cos \left(\frac{2 \pi j}{n}\right)}\right)
$$

should be equal to zero, up to second-order terms. Then, from (3.22), (3.59) and $(3.60)$,

with

$$
\mathrm{D}(\Re)=-\frac{1}{2 \pi n}\left[\frac{1-\cos \left(\frac{2 \pi j}{n}\right)}{\sin \left(\frac{2 \pi j}{n}\right)} \log \left(2 \sin \left(\frac{\pi j}{n}\right)\right)\right]
$$

$$
\operatorname{tl}(\Re)=+\frac{1}{n} O\left(\left(\frac{\log \log n}{\log n}\right)^{2}\right),
$$

and with the constant $\tan (\pi j / n)$ in the Big O. An uniform upper bound of all $\tan (\pi j / n)$ is $\tan (\pi / 4)=1$.

Let us now check the validity of the assumption on

$$
2 \pi \Re \sin \left(\frac{2 \pi j}{n}\right) /\left(1-\cos \left(\frac{2 \pi j}{n}\right)\right):
$$

using (3.58) in the summation (3.60) set equal to 0 , we readily obtain

$$
+2 \pi \Re\left(\frac{\sin \left(\frac{2 \pi j}{n}\right)}{1-\cos \left(\frac{2 \pi j}{n}\right)}\right) \cong+\frac{1}{2 \pi^{2}} \frac{\log \log n}{(\log n)^{2}}
$$

for $n$ large enough, uniformly in $j$, for all the roots $z_{j, n}$ in this sector.

To finish up the proof of Proposition 3.4 we have to provide the expansions of the real and imaginary parts of the roots $z_{j, n}$ as functions of $\Re$ and $\Im$. From (3.30), taking the expansions of $\Re$ and $\Im$, we deduce

$$
\Re\left(z_{j, n}\right)=\cos \left(\frac{2 \pi j}{n}\right)\left[\theta_{n}-2 \pi \mathrm{D}(\Im)\right]-\sin \left(\frac{2 \pi j}{n}\right)(2 \pi \mathrm{D}(\Re))+\cdots
$$

and 


$$
\Im\left(z_{j, n}\right)=\sin \left(\frac{2 \pi j}{n}\right)\left[\theta_{n}-2 \pi \mathrm{D}(\Im)\right]+\cos \left(\frac{2 \pi j}{n}\right)(2 \pi \mathrm{D}(\Re))+\cdots
$$

We now replace $\mathrm{D}(\Re)$ and $\mathrm{D}(\Im)$ by their respective expressions in (3.63) and (3.64). The expressions of $\mathrm{D}\left(\Re\left(z_{j, n}\right)\right)$ and $\mathrm{D}\left(\Im\left(z_{j, n}\right)\right)$ follow as functions of the two variables $n$ and $j / n$.

Proposition 3.5. The roots $z_{j, n}$ of $G_{n}(X), n \geq n_{0}=18$, which belong to the angular sector (with the convention taken in Remark 3.3) $\frac{\pi}{2}>\arg z>2 \pi \frac{\log n}{n}$, have a modulus whose asymptotic expansion is

$$
\left|z_{j, n}\right|=1+\frac{1}{n} \log \left(2 \sin \left(\frac{\pi j}{n}\right)\right)+\frac{1}{n} O\left(\left(\frac{\log \log n}{\log n}\right)^{2}\right),
$$

where the constant involved in $O()$ in the tail does not depend upon $j$.

Pr o of. For the roots $z_{j, n}$ in this sector, since

$$
\left|z_{j, n}\right|=\theta_{n}-2 \pi \mathrm{D}(\Im)+\cdots
$$

by (3.30) and that $(\log n)\left[1-\lambda_{n}\right]=n\left(1-\mathrm{D}\left(\theta_{n}\right)\right)$ we readily obtain (3.65), from (3.22), (3.23), (3.55) and (3.56).

Proposition 3.5 will be used in particular to study the geometry of the roots which lie in a small neighbourhood of $e^{i \pi / 3}$.

Corollary 3.6. Let $\psi_{j, n}:=\arg \left(z_{j, n}\right) \in(0, \pi / 3)$ for $1 \leq j \leq\lfloor n / 6\rfloor$. Then, for $j / n$ close to $1 / 6$ and $n$ large enough, $\psi_{j, n}$ admits the following (two variables-) asymptotic expansion

$$
\begin{aligned}
\psi_{j, n}=\frac{\pi}{3} & -2 \pi\left(1-\frac{1}{2 n}\right)\left(\frac{1}{6}-\frac{j}{n}\right)-\frac{2 \sqrt{3} \pi^{2}}{3 n}\left(\frac{1}{6}-\frac{j}{n}\right)^{2} \\
+ & \frac{1}{n} O\left(\left(\frac{1}{6}-\frac{j}{n}\right)^{3}\right)+\frac{1}{n} O\left(\left(\frac{\log \log n}{\log n}\right)^{2}\right) .
\end{aligned}
$$

P r o o f. By Proposition 3.4 (ii), more precisely using (3.30), (3.61), (3.62), since

$$
\log \left(2 \sin \left(\frac{\pi j}{n}\right)\right)=-\sqrt{3} \pi\left(\frac{1}{6}-\frac{j}{n}\right)-2 \pi^{2}\left(\frac{1}{6}-\frac{j}{n}\right)^{2}+O\left(\left(\frac{1}{6}-\frac{j}{n}\right)^{3}\right),
$$

we readily obtain the asymptotic expansion of $\psi_{j, n}$ in the two variables " $j / n$ " and " $n$ ":

$$
\psi_{j, n}=2 \pi\left(\frac{j}{n}-\frac{1}{2 \pi n} \frac{\sin \left(\frac{\pi j}{n}\right)}{\cos \left(\frac{\pi j}{n}\right)} \log \left(2 \sin \left(\frac{\pi j}{n}\right)\right)\right)+\frac{1}{n} O\left(\left(\frac{\log \log n}{\log n}\right)^{2}\right)=\frac{2 \pi j}{n}
$$




$$
+\frac{\pi}{n}\left(\frac{1}{6}-\frac{j}{n}\right)-\frac{2 \sqrt{3} \pi^{2}}{3 n}\left(\frac{1}{6}-\frac{j}{n}\right)^{2}+\frac{1}{n} O\left(\left(\frac{1}{6}-\frac{j}{n}\right)^{3}\right)+\frac{1}{n} O\left(\left(\frac{\log \log n}{\log n}\right)^{2}\right) .
$$

Up to the error terms $\psi_{j, n}$ behaves as $\frac{2 \pi j}{n}$. From (3.68) we deduce (3.66).

Proposition 3.7. Let $n \geq 2$. Then (i) the number $p_{n}$ of roots of $G_{n}(X)$ which lie inside the open sector $\mathcal{S}=\{z|| \arg (z) \mid<\pi / 3\}$ is equal to

$$
1+2\left\lfloor\frac{n}{6}\right\rfloor
$$

(ii) the correlation between the geometry of the roots of $G_{n}(X)$ which lie inside the unit disc and the upper half-plane and their indexation is given by

$$
j \in\left\{1,2, \ldots,\left\lfloor\frac{n}{6}\right\rfloor\right\} \Longleftrightarrow \Re\left(z_{j, n}\right)>\frac{1}{2} \Longleftrightarrow\left|z_{j, n}\right|<1,
$$

and the Mahler measure $\mathrm{M}\left(G_{n}\right)$ of the trinomial $G_{n}(X)$ is

$$
\mathrm{M}\left(G_{n}\right)=\mathrm{M}\left(G_{n}^{*}\right)=\theta_{n}^{-1} \prod_{j=1}^{\lfloor n / 6\rfloor}\left|z_{j, n}\right|^{-2} .
$$

Proof. The formula $p_{n}=1+2\left\lfloor\frac{n}{6}\right\rfloor$ is true for $n=2$ to 51 as it can be easily checked. For larger $n$ we now prove it recursively.

First let us observe that the open unit $\operatorname{disc}\{z \in \mathbb{C}|| z-1 \mid<1\}$ centred at 1 contains all the roots of $G_{n}(X)$ of modulus $<1$ since

$$
\left|z_{j, n}\right|<1 \Longleftrightarrow\left|z_{j, n}\right|^{n}=\left|1-z_{j, n}\right|<1 \text {. }
$$

Then it suffices to understand the geometry of the roots in a neighbourhood of $e^{i \pi / 3}$. The integer $j$ is $\geq 1$ in the sequel. Let us show the following equivalences

$$
\arg \left(z_{j, n}\right)<\pi / 3 \Longleftrightarrow\left|z_{j, n}\right|<1 \Longleftrightarrow \Re\left(z_{j, n}\right)>\frac{1}{2} .
$$

For proving (3.73) we make use of the expansions $\mathrm{D}\left(\Re\left(z_{j, n}\right)\right)$ and $\mathrm{D}\left(\Im\left(z_{j, n}\right)\right)$ for $z_{j, n}$ in a neighbourhood of $e^{i \pi / 3}$, belonging to the main sector " $\pi / 2>\arg z>$ $2 \pi \log n / n "$ (Proposition 3.4 (ii)): since

$$
\begin{aligned}
\Re\left(z_{j, n}\right) & =\cos \left(\frac{2 \pi j}{n}\right)+\frac{\log \left(2 \sin \left(\frac{\pi j}{n}\right)\right)}{n}+\cdots \\
& =\cos \left(\left(\frac{2 \pi j}{n}-\frac{\pi}{3}\right)+\frac{\pi}{3}\right)+\frac{\left.\log \left(2 \sin \left(\left(\frac{\pi j}{n}-\frac{\pi}{6}\right)+\frac{\pi}{6}\right)\right)\right)}{n}+\cdots \\
& =\frac{1}{2}-\frac{\sqrt{3}}{2}\left(\frac{2 \pi j}{n}-\frac{\pi}{3}\right)+\cdots,
\end{aligned}
$$




\section{JEAN-LOUIS VERGER-GAUGRY}

we obtain the equivalence

$$
\arg \left(z_{j, n}\right)<\pi / 3 \Longleftrightarrow \Re\left(z_{j, n}\right)>\frac{1}{2}
$$

when $n \not \equiv 0 \quad(\bmod 6)$, and it holds, in this case, if and only if $\frac{2 \pi j}{n}-\frac{\pi}{3}<0$, that is $j \leq\lfloor n / 6\rfloor$. By Proposition 3.5,

$$
\left|z_{j, n}\right|=1+\frac{1}{n} \log \left(2 \sin \left(\frac{\pi j}{n}\right)\right)+\frac{1}{n} O\left(\left(\frac{\log \log n}{\log n}\right)^{2}\right) .
$$

Since, for $x \in[-1 / 2,+1 / 2], \log (2|\sin (\pi x)|)<0 \Longleftrightarrow|x|<1 / 6$, we deduce that the inequality $\left|z_{j, n}\right|<1$ is approximately equivalent to $\frac{\pi j}{n}<\frac{\pi}{6}$, i.e., $j$ approximately less than $n / 6$. Obviously it is equivalent to $j \leq\lfloor n / 6\rfloor$ when $n \not \equiv 0 \quad(\bmod 6)$, and therefore (3.73) holds if and only if $1 \leq j \leq\lfloor n / 6\rfloor$ when $n \not \equiv 0 \quad(\bmod 6)$. Let us now prove that $(3.73)$ is equivalent to $1 \leq j \leq\lfloor n / 6\rfloor$ for all integers $n \geq 2$.

By Proposition 2.3 the trinomial $G_{n}(X)$ admits $e^{ \pm i \pi / 3}=\frac{1}{2} \pm i \frac{\sqrt{3}}{2}$ as roots if and only if $n \equiv 5 \quad(\bmod 6)$. For $n \equiv 5(\bmod 6)$ the root $e^{i \pi / 3}$ is necessarily one of the roots $z_{j, n}$ for which $j$ is close to $n / 6$, by Proposition 3.4 (ii), whose real part is

$$
\mathrm{D}\left(\Re\left(z_{j, n}\right)\right)=\cos \left(\frac{2 \pi j}{n}\right)+\frac{\log \left(2 \sin \left(\frac{\pi j}{n}\right)\right)}{n} .
$$

Therefore, if $n=6 q+5$, with $q \geq 1$, the corresponding value of $j$ is $q+1$, so that

$$
z_{q+1,6 q+5}=z_{q+2,6 q+11}=e^{i \pi / 3} \quad \text { for all } q \geq 0 .
$$

Let us assume that $p_{6 q+5}=1+2\left\lfloor\frac{6 q+5}{6}\right\rfloor$ (with $q \geq 1$ ) holds and let us prove that the formula $p_{6 q+6}=1+2\left\lfloor\frac{6 q+6}{6}\right\rfloor$ also holds. From Proposition 3.4 (see (3.68)) the distance between two successive roots of $G_{n}(X)$ in $\Re(z)>0$ is $=2 \pi / n+\cdots$, and therefore, when $j$ is fixed, the $j$ th root $z_{j, n}$ "rotates to the right towards 1 along the unit circle", to become the $j$ th root $z_{j, n+1}$ of $G_{n+1}(X)$. Thus the number of roots of $G_{6 q+5+1}(X)$ within the sector $\mathcal{S}$ is the number of roots of $G_{6 q+5}(X)$ within $\mathcal{S}$ plus 2 , and $p_{6 q+6}=1+2\left\lfloor\frac{6 q+6}{6}\right\rfloor=1+2\left\lfloor\frac{6 q+5}{6}\right\rfloor+2$ is also true.

Let us continue the recursion. For $n=6 q+m$ and $m=6$ to 11 , when $n$ is increased by 1 to $n+1$, the collection of roots $\left\{z_{j, n}\right\}$ which lie near $e^{i \pi / 3}$ slightly rotates to the right to become the collection $\left\{z_{j, n+1}\right\}$. Using the expressions of $\mathrm{D}\left(\Re\left(z_{j, n}\right)\right)$ in Proposition 3.4, for $j$ close to $n / 6$, we deduce 
CONJECTURE OF LEHMER, ASYMPTOTIC EXPANSIONS OF THE MAHLER MEASURE

$$
\begin{aligned}
\Re\left(z_{q+2,6 q+5}\right) & \\
<\Re\left(z_{q+2,6 q+6}\right) & <\Re\left(z_{q+2,6 q+7}\right)<\Re\left(z_{q+2,6 q+8}\right)<\Re\left(z_{q+2,6 q+9}\right) \\
<\Re\left(z_{q+2,6 q+10}\right) & <\frac{1}{2}=\Re\left(z_{q+2,6 q+11}\right)=\Re\left(z_{q+1,6 q+5}\right)<\Re\left(z_{q+1,6 q+6}\right) \\
& <\Re\left(z_{q+1,6 q+7}\right)<\Re\left(z_{q+1,6 q+8}\right)<\Re\left(z_{q+1,6 q+9}\right)<\Re\left(z_{q+1,6 q+10}\right) .
\end{aligned}
$$

Counting the complex conjugates, these inequalities show that

$$
p_{6 q+6}=p_{6 q+7}=p_{6 q+8}=p_{6 q+9}=p_{6 q+10}=p_{6 q+11} .
$$

We deduce the general formula (3.69) for $p_{n}$, and the equivalences (3.70) and (3.73), for all integers $n \geq 2$.

To obtain (3.71) we first invoke the general fact that $\mathrm{M}\left(G_{n}^{*}\right)=\mathrm{M}\left(G_{n}\right)$ for $n \geq 2$. Then the roots of $G_{n}^{*}(X)$ which possess a real part $>1 / 2$ are those which lie outside the closed unit disc. These roots are the inverses of those of $G_{n}(X)$ which lie inside the unit disc, characterized by (3.70). The expression (3.71) of $\mathrm{M}\left(G_{n}\right)$ follows; it is valid for $n$ odd or $n$ even, whatever the form (2.15) the trinomial $G_{n}(X)$ takes.

Proposition 3.8. Let $n \geq 6$. The roots of modulus $<1$ of $G_{n}(z)$ in the closed upper half-plane have the following properties:

(i) $\theta_{n}<\left|z_{1, n}\right|$,

(ii) for any pair of successive indices $j, j+1$ in $\{1,2, \ldots,\lfloor n / 6\rfloor\}$,

$$
\left|z_{j, n}\right|<\left|z_{j+1, n}\right|
$$

P r o o f. (i) is proved in [FLP]. Let us prove (ii). Assume the contrary: $\left|z_{j+1, n}\right|<$ $\left|z_{j, n}\right|$ for a certain $j$ in $\{1,2, \ldots,\lfloor n / 6\rfloor-1\}$. Then

$$
-1+z_{j, n}+z_{j, n}^{n}=-1+z_{j+1, n}+z_{j+1, n}^{n} .
$$

We deduce

$$
\frac{\left|z_{j+1, n}\right|}{\left|z_{j, n}\right|}=\frac{\left|1+z_{j, n}^{n-1}\right|}{\left|1+z_{j+1, n}^{n-1}\right|} .
$$

The assumption $\left|z_{j+1, n}\right|<\left|z_{j, n}\right|$ implies that the ratio $\frac{\left|z_{j+1, n}\right|}{\left|z_{j, n}\right|}$ is $<1$.

Now there are two cases: either (ii-a) $j$ and $j+1$ together are such that $z_{j, n}$ and $z_{j+1, n}$ belong to the "bump" sector (i.e., for which $j / \log n=o(1)$ ) or to the "main sector" (i.e., for which $(\log n) / j=o(1))$, (the two cases (i) and (ii) of Proposition 3.4), or (ii-b) $j$ and $j+1$ are both such that $z_{j, n}$ and $z_{j+1, n}$ in the transition region $j \asymp \log n$. 
In the first case (ii-a), from Proposition 3.4, we deduce from the asymptotic expansion of $z_{j, n}$ that $\arg \left(z_{j, n}\right)$ is equal to $\frac{2 i \pi j}{n}+\cdots$ Therefore $\arg \left(z_{j, n}^{n-1}\right) \cong$ $-\arg \left(z_{j, n}\right)=\arg \left(z_{-j, n}\right)$ and $z_{j, n}^{n-1} \cong\left|z_{j, n}\right|^{n-1} e^{-2 i \pi j / n}$. The enumeration of the roots $\left(z_{-j, n}\right)_{j}$ by the index $-j$ is clockwise and, by Proposition 3.7, both real parts $\Re\left(\left|z_{j, n}\right|^{n-1} e^{-2 i \pi j / n}\right)$ and $\Re\left(\left|z_{j+1, n}\right|^{n-1} e^{-2 i \pi(j+1) / n}\right)$ are $>1 / 2$. Moreover, the assumption $\left|z_{j+1, n}\right|<\left|z_{j, n}\right|$ implies $\left|z_{j+1, n}^{n-1}\right|<\left|z_{j, n}^{n-1}\right|$. Therefore, assuming $\left|z_{j+1, n}\right|<\left|z_{j, n}\right|$ implies that the distance $\left|1+z_{j, n}^{n-1}\right|$ between -1 and $z_{j, n}^{n-1}$ is larger than the distance $\left|1+z_{j+1, n}^{n-1}\right|$ between -1 and $z_{j+1, n}^{n-1}$. Contradiction with (3.74) since the ratio $\left|1+z_{j, n}^{n-1}\right| /\left|1+z_{j+1, n}^{n-1}\right|$ is $<1$.

In the second case (ii-b), using the notations of Remark 3.3, the two successive roots $z_{j, n}$ and $z_{j+1, n}$ are such that $j \asymp \log n$, i.e.,

$$
\frac{2 i \pi u_{n}}{n}<\arg \left(z_{j, n}\right)<\arg \left(z_{j+1, n}\right)<\frac{2 i \pi\left(v_{n}+1\right)}{n}
$$

with

$$
\Re\left(z_{j, n}\right)>1 / 2 \text { and } \Re\left(z_{j+1, n}\right)>1 / 2 .
$$

Therefore,

$$
\frac{-2 i \pi u_{n}}{n}>\arg \left(z_{j, n}^{n-1}\right)>\arg \left(z_{j+1, n}^{n-1}\right)>\frac{-2 i \pi\left(v_{n}+1\right)}{n}
$$

for which $\lim _{n \rightarrow \infty} u_{n} / n=\lim _{n \rightarrow \infty} v_{n} / n=0$. Hence

$$
\Re\left(z_{j, n}^{n-1}\right)>1 / 2 \text { and } \Re\left(z_{j+1, n}^{n-1}\right)>1 / 2 .
$$

We can now conclude as in (ii-a). Assuming $\left|z_{j+1, n}\right|<\left|z_{j, n}\right|$ implies that the distance $\left|1+z_{j, n}^{n-1}\right|$ between -1 and $z_{j, n}^{n-1}$ is larger than the distance $\left|1+z_{j+1, n}^{n-1}\right|$ between -1 and $z_{j+1, n}^{n-1}$. Therefore we would have $\left|1+z_{j, n}^{n-1}\right| /\left|1+z_{j+1, n}^{n-1}\right|>1$, which is a contradiction with (3.74).

\section{Limit Mahler measure : proof of Theorem 1.1}

\subsection{Using bivariate Mahler measures}

The Mahler measure of a polynomial $f(X, Y)$ in two variables is defined by

$$
\mathrm{M}(f(X, Y))=\exp \left(\int_{0}^{1} \int_{0}^{1} \log \left|f\left(e^{2 \pi i s}, e^{2 \pi i t}\right)\right| \mathrm{d} s \mathrm{~d} t\right) .
$$

Boyd [Bo3], [Lw] ([Bo1, p 130]; [Bo2, in Lemma 1]) prove that the Mahler measure of a polynomial in two variables $f(X, Y)$ is the limit of the Mahler measures 
of polynomials in one variable obtained by replacing the second variable by a power of the first one

$$
\mathrm{M}(f(X, Y))=\lim _{n \rightarrow \infty} \mathrm{M}\left(f\left(X, X^{n}\right)\right) .
$$

Since

$$
\begin{aligned}
\mathrm{M}\left(G_{n}\right) & =\exp \left(\frac{1}{2 \pi} \int_{0}^{2 \pi} \log \left|-1+e^{i t}+e^{i n t}\right| \mathrm{d} t\right), \\
\lim _{n \rightarrow+\infty} \log \left(\mathrm{M}\left(G_{n}\right)\right) & =\left(\frac{1}{2 \pi}\right)^{2} \int_{0}^{2 \pi} \mathrm{d} s \int_{0}^{2 \pi} \log \left|e^{i t}+e^{i s}-1\right| \mathrm{d} t .
\end{aligned}
$$

From Theorem 2 in Smyth [Sy2] and by Jensen's formula

$$
\begin{aligned}
\frac{3 \sqrt{3}}{4 \pi} \mathrm{L}\left(2, \chi_{3}\right) & =\log \mathrm{M}(-1+X+Y)=\log \mathrm{M}(\max \{|-1+X|, 1\}) \\
& =\frac{1}{\pi} \int_{0}^{\pi} \log ^{+}\left|-1+e^{i t}\right| \mathrm{d} t=\frac{-1}{\pi} \int_{0}^{\pi / 3} \log (2 \sin (t / 2)) \mathrm{d} t \\
& =\log (1.38135 \ldots) .
\end{aligned}
$$

This value is given by the Bloch-Wigner dilogarithm [BM] [Ln].

\subsection{Using asymptotic expansions}

In the case where $G_{n}$ is not irreducible, i.e., for $n \equiv 5 \bmod 6$, the Mahler measure $\mathrm{M}\left(G_{n}\right)$ of $G_{n}(X)$ is equal to the Mahler measure

$$
\mathrm{M}\left(G_{n}(X) /\left(X^{2}-X+1\right)\right)
$$

by Proposition 2.3. We will consider the two cases " $G_{n}$ irreducible" and " $G_{n}$ reducible" simultaneously, not taking care of reducibility. Indeed, the roots of $X^{2}-X+1$ lie on the unit circle, and the indexation of the roots of $G_{n}$ which is chosen, with $j$, in Proposition 3.7, is such that the maximal value $\lfloor n / 6\rfloor$ of the index $j$ involves both cases.

First let us observe that the Riemann-Stieljes sum

$$
S(f, n):=-2 \sum_{j=1}^{\lfloor n / 6\rfloor} \frac{1}{n} \log \left(2 \sin \left(\frac{\pi j}{n}\right)\right)=\frac{-1}{\pi} \sum_{j=1}^{\lfloor n / 6\rfloor}\left(x_{j}-x_{j-1}\right) f\left(x_{j}\right)
$$

with $x_{j}=\frac{2 \pi j}{n}$ and $f(x):=\log \left(2 \sin \left(\frac{x}{2}\right)\right)$ converges to the limit

$$
\lim _{n \rightarrow \infty} S(f, n)=\frac{-1}{\pi} \int_{0}^{\pi / 3} f(x) d x=\log \Lambda=\log (1.38135 \ldots) .
$$


From Proposition 3.7 we have

$$
\log \mathrm{M}\left(G_{n}\right)=-\log \left(\theta_{n}\right)-2 \sum_{j=1}^{\lfloor n / 6\rfloor} \log \left|z_{j, n}\right| .
$$

Since $\lim _{n_{\infty}} \theta_{n}=1$, by Proposition 3.1, then $\lim _{n_{\infty}} \log \theta_{n}=0$. Therefore we will just show that

$$
\lim _{n \rightarrow \infty} S(f, n)=\lim _{n \rightarrow \infty} \log \mathrm{M}\left(G_{n}\right)=-2 \lim _{n \rightarrow \infty} \sum_{j=1}^{\lfloor n / 6\rfloor} \log \left|z_{j, n}\right| .
$$

by a suitable decomposition of the summation in (4.75). In this summation the asymptotic expansion of $\log \left|z_{j, n}\right|$ takes different forms, by Proposition 3.4 and Proposition 3.5, according to the angular sector to which $z_{j, n}$ belongs, i.e., to the asymptotic behaviour of $j / n$ or $j / \log n$ (cf. Remark 3.3).

For $n \geq n_{0}=18$, the decomposition into angular sectors between $\pi / 3$ and 0 follows from Remark 3.3, as:

$$
\frac{\pi}{3} \geq 2 \pi \frac{v_{n}}{n}>2 \pi \frac{2 \log n-v_{n}}{n}>2 \pi \frac{u_{n}}{n}>2 \pi \frac{2 \sqrt{(\log n)(\log \log n)}-u_{n}}{n}>0 .
$$

The corresponding decomposition of the summation $\sum_{j=1}^{\lfloor n / 6\rfloor}$ in (4.76) is

$$
\begin{gathered}
=\sum_{j=1}^{\left\lfloor 2 \sqrt{(\log n)(\log \log n)}-u_{n}\right\rfloor}+\sum_{\left\lceil 2 \sqrt{(\log n)(\log \log n)}-u_{n}\right\rceil}^{\left\lfloor u_{n}\right\rfloor} \\
+\sum_{j=\left\lceil u_{n}\right\rceil}^{\left\lfloor 2 \log n-v_{n}\right\rfloor}+\sum_{\left\lceil 2 \log n-v_{n}\right\rceil}^{\left\lfloor v_{n}\right\rfloor}+\sum_{j=\left\lceil v_{n}\right\rceil}^{\lfloor n / 6\rfloor}
\end{gathered}
$$

We now show that, in (4.77), the last summation $\sum_{j=\left\lceil v_{n}\right\rceil}^{\lfloor n / 6\rfloor}$ is asymptotically the dominant one and that the other summations tend to zero when $n$ goes to infinity.

Main sector, dominant contribution: $\pi / 3>\arg z>2 \pi \frac{\log n}{n}$ :

From (3.65) we have

$$
\sum_{j=\left\lceil v_{n}\right\rceil}^{\lfloor n / 6\rfloor} \log \left|z_{j, n}\right|=\sum_{j=\left\lceil v_{n}\right\rceil}^{\lfloor n / 6\rfloor} \log \left(1+\frac{1}{n} \log \left(2 \sin \left(\frac{\pi j}{n}\right)\right)+\frac{1}{n} O\left(\left(\frac{\log \log n}{\log n}\right)^{2}\right)\right) .
$$


By the remainder Theorem of alternating series let us recall that, for $x$ a real number, $|x|<1$, the inequality $|\log (1+x)-x| \leq \frac{x^{2}}{2}$ holds. Then

$$
\begin{aligned}
& \left|\sum_{j=\left\lceil v_{n}\right\rceil}^{\lfloor n / 6\rfloor} \log \right| z_{j, n}\left|-\sum_{j=\left\lceil v_{n}\right\rceil}^{\lfloor n / 6\rfloor} \frac{1}{n} \log \left(2 \sin \left(\frac{\pi j}{n}\right)\right)\right| \leq \sum_{j=\left\lceil v_{n}\right\rceil}^{\lfloor n / 6\rfloor} \frac{1}{n}\left|O\left(\left(\frac{\log \log n}{\log n}\right)^{2}\right)\right| \\
& \quad+\frac{1}{2} \sum_{j=\left\lceil v_{n}\right\rceil}^{\lfloor n / 6\rfloor} \frac{1}{n^{2}}\left[\log \left(2 \sin \left(\frac{\pi j}{n}\right)\right)+O\left(\left(\frac{\log \log n}{\log n}\right)^{2}\right)\right]^{2}
\end{aligned}
$$

For $1 \leq j \leq\lfloor n / 6\rfloor$, the inequalities $0<2 \sin (\pi j / n) \leq 1$ and $\log (2 \sin (\pi j / n))<0$ hold. Then $|\log (2 \sin (\pi j / n))| \leq|\log (2 \sin (\pi / n))|=O(\log n)$. On the other hand, by Proposition 3.5 the two $O($ )s in the rhs of the inequality (4.78) involve a constant which does not depend upon $j$. Therefore the rhs of (4.78) is

$$
=O\left(\left(\frac{\log \log n}{\log n}\right)^{2}\right)+O\left(\frac{\log ^{2} n}{n}\right)=O\left(\left(\frac{\log \log n}{\log n}\right)^{2}\right) \text {. }
$$

From (4.78), using (3.29), we deduce

$$
\begin{aligned}
& \left|\sum_{j=\left\lceil v_{n}\right\rceil}^{\lfloor n / 6\rfloor} \log \right| z_{j, n}\left|-\sum_{j=1}^{\lfloor n / 6\rfloor} \frac{1}{n} \log \left(2 \sin \left(\frac{\pi j}{n}\right)\right)\right| \\
& \leq \sum_{j=1}^{\left\lfloor u_{n}\right\rfloor} \frac{1}{n}\left|\log \left(2 \sin \left(\frac{\pi j}{n}\right)\right)\right|+\sum_{j=\left\lceil u_{n}\right\rceil}^{\left\lfloor v_{n}\right\rfloor} \frac{1}{n}\left|\log \left(2 \sin \left(\frac{\pi j}{n}\right)\right)\right|+O\left(\left(\frac{\log \log n}{\log n}\right)^{2}\right) \\
& =O\left(\frac{\log ^{2} n}{n}\right)+O\left(\frac{\log ^{2+\epsilon} n}{n}\right)+O\left(\left(\frac{\log \log n}{\log n}\right)^{2}\right)=O\left(\left(\frac{\log \log n}{\log n}\right)^{2}\right) .
\end{aligned}
$$

Moreover, let us observe that: $\lim _{n \rightarrow+\infty} \frac{2 \pi}{n}\left\lfloor\frac{n}{6}\right\rfloor=\pi / 3$. Hence

$$
\lim _{n \rightarrow \infty} S(f, n)=\lim _{n \rightarrow \infty}(-2) \sum_{j=\left\lceil v_{n}\right\rceil}^{\lfloor n / 6\rfloor} \log \left|z_{j, n}\right| .
$$

"Bump sector", the other contributions in (4.77):

Instead of considering the four other summations of (4.77) independently, it will be sufficient to gather the roots involved into these summations into the following two subcollections indexed by $j$, as:

- $1 \leq j \leq\left\lfloor u_{n}\right\rfloor$, and

- $\left\lceil u_{n}\right\rceil \leq j \leq\left\lfloor v_{n}\right\rfloor$. 


\section{JEAN-LOUIS VERGER-GAUGRY}

Case (i): for all $j$ satisfying $1 \leq j \leq\left\lfloor u_{n}\right\rfloor$, by taking the first terms in the

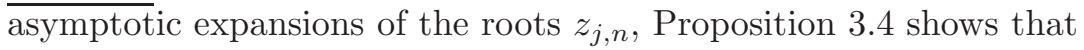

$$
\mathrm{D}\left(\Im\left(z_{j, n}\right)\right)=\frac{2 \pi j}{n}+\frac{1}{n} O\left(\frac{u_{n}}{\log n}\right),
$$

with a $j$-independent constant involved in $O()$. From Proposition 3.5 the asymptotic expansions of the moduli $\left|z_{j, n}\right|$ are given by

$$
\left|z_{j, n}\right|=\theta_{n}-2 \pi \mathrm{D}\left(\Im\left(z_{j, n}\right)\right)+\cdots
$$

By the remainder Theorem of alternating series and Proposition 3.1 we deduce

$$
\begin{aligned}
\log \left|z_{j, n}\right|= & \left(\theta_{n}-1\right)-2 \pi \mathrm{D}\left(\Im\left(z_{j, n}\right)\right) \\
& +\frac{1}{n} O\left(\frac{u_{n}}{\log n}\right)+O\left(\left(\left(\theta_{n}-1\right)-2 \pi \mathrm{D}\left(\Im\left(z_{j, n}\right)\right)\right)^{2}\right) \\
= & -\frac{\log n}{n}-\frac{4 \pi^{2} j}{n}+O\left(\frac{\log \log n}{n}\right) \\
& +\frac{1}{n} O\left(\frac{u_{n}}{\log n}\right)+O\left(\left(\frac{\log n}{n}\right)^{2}\right) .
\end{aligned}
$$

Hence,

$$
\begin{aligned}
\sum_{j=1}^{\left\lfloor u_{n}\right\rfloor} \log \left|z_{j, n}\right|= & -\frac{\left\lfloor u_{n}\right\rfloor \log n}{n}-\frac{\left\lfloor u_{n}\right\rfloor\left(\left\lfloor u_{n}\right\rfloor\right) 2 \pi^{2}}{n} \\
& +O\left(\frac{\left\lfloor u_{n}\right\rfloor(\log \log n)}{n}\right)=O\left(\frac{\log ^{2} n}{n}\right) .
\end{aligned}
$$

Therefore,

$$
\lim _{n \rightarrow \infty} \sum_{j=1}^{\left\lfloor u_{n}\right\rfloor} \log \left|z_{j, n}\right|=0 .
$$

$C$ ase (ii) : for all $j$ satisfying " $\left\lceil u_{n}\right\rceil \leq j \leq\left\lfloor v_{n}\right\rfloor$ ", Proposition 3.4 does not provide

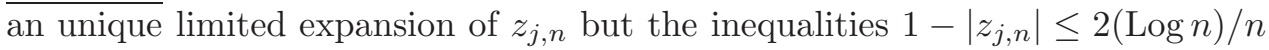
hold by Lemma 2.1. We deduce

$$
\begin{aligned}
\left|\sum_{j=\left\lceil u_{n}\right\rceil}^{\left\lfloor v_{n}\right\rfloor} \log \right| z_{j, n}|| & \leq\left(v_{n}-u_{n}\right) \frac{2 \log n}{n}+O\left(\left(v_{n}-u_{n}\right) \frac{\log ^{2} n}{n^{2}}\right) \\
& =O\left(\frac{\log ^{2+\epsilon} n}{n}\right),
\end{aligned}
$$


and the limit

$$
\lim _{n \rightarrow \infty} \sum_{j=\left\lceil u_{n}\right\rceil}^{\left\lfloor v_{n}\right\rfloor} \log \left|z_{j, n}\right|=0 .
$$

To finish up the proof of Theorem 1.1 we take the exponential of (4.76).

\section{Asymptotics of the Mahler measure}

\subsection{Second-order terms for the moduli of the roots}

The second-order terms in the asymptotic expansions of the moduli $\left|z_{j, n}\right|$ of the roots $z_{j, n}$ of $G_{n}$ are required for obtaining the asymptotic speed of convergence of the Mahler measure $\mathrm{M}\left(G_{n}\right)$ towards the limit Mahler measure $\Lambda$. From Subsection 4.2 it is sufficient to obtain them for the roots lying in the main sector: $\pi / 3>\arg z>2 \pi \frac{\log n}{n}$, the other roots lying in the "bump" sector contributing negligibly to the Mahler measure. The expansions used for obtaining (3.65) have to be improved; the first step is to expand further (3.53) by taking into account the term $\lambda_{n}^{2} \cong\left(\frac{\log \log n}{\log n}\right)^{2}\left(1-\frac{2}{\log n}\right)$, from (3.28). Then (3.55) and (3.56) are replaced by

$$
\mathrm{D}(\Im)=\frac{-1}{2 \pi n}\left[(\log n)\left(1-\lambda_{n}\right)+\frac{1}{2}\left(\frac{\log \log n}{\log n}\right)^{2}+\log \left(2 \sin \left(\frac{\pi j}{n}\right)\right)\right]
$$

with

$$
\operatorname{tl}(\Im)=+\frac{1}{n} O\left(\frac{(\log \log n)^{2}}{(\log n)^{3}}\right)
$$

with the constant $1 /(2 \pi)$ involved in the Big O, independently of $j$. Then, for $\pi / 3 \geq \arg z_{j, n}>2 \pi \frac{\left\lceil v_{n}\right\rceil}{n}$, (3.65) is expanded further as

$$
\left|z_{j, n}\right|=\mathrm{D}\left(\left|z_{j, n}\right|\right)+\mathrm{tl}\left(\left|z_{j, n}\right|\right)
$$

with

$$
\mathrm{D}\left(\left|z_{j, n}\right|\right)=1+\frac{1}{n} \log \left(2 \sin \left(\frac{\pi j}{n}\right)\right)+\frac{1}{2 n}\left(\frac{\log \log n}{\log n}\right)^{2}
$$

and

$$
\operatorname{tl}\left(\left|z_{j, n}\right|\right)=\frac{1}{n} O\left(\frac{(\log \log n)^{2}}{(\log n)^{3}}\right),
$$

where the constant involved in $O($ ) is 1 (does not depend upon $j$ ). 


\subsection{Minoration of the Mahler measure and proof of Theorem 1.2}

In 1933, in the search of big prime numbers, Lehmer [Le] asked the following problem: if $\epsilon$ is a positive quantity, to find a polynomial of the form

$$
f(x)=x^{r}+a_{1} x^{r-1}+\cdots+a_{r}
$$

where the a's are integers, such that the absolute value of the product of those roots of $f$ which lie outside the unit circle, lies between 1 and $1+\epsilon \ldots$ Whether or not the problem has a solution for $\epsilon<0.176$ we do not know.

Lehmer's Conjecture was initially addressed to nonzero algebraic integers which are not roots of unity. Today (2015), the smallest known Mahler measure of nonzero algebraic numbers which are not roots of unity is the one discovered by Lehmer [Le], a Salem number (Lehmer's number)

$$
\mathrm{M}\left(X^{10}+X^{9}-X^{7}-X^{6}-X^{5}-X^{4}-X^{3}+X+1\right)=1.1762 \ldots
$$

Exhaustive search [Sy3] for the smallest Mahler measures was carried out by Boyd, Poulet, Mossinghoff, Lisonek, Flammang, Grandcolas, Rhin, Sac-Epée. The list of Mossinghoff [MfL] gives primitive, irreducible, noncyclotomic integer polynomials with degree at most 180 having Mahler measure less than 1.3, and this list is complete through degree 44 (Mossinghoff, Rhin and $\mathrm{Wu}$ [MRW]).

For $\alpha$ an algebraic integer of degree $d>1$, not a root of unity, Blansky and Montgomery $[\mathrm{ByM}]$ showed, with multivariate Fourier series,

$$
\mathrm{m}(\alpha)>1+\frac{1}{52} \frac{1}{d \log (6 d)} .
$$

By a different approach, using an auxiliary function and a proof of transcendence (Thue's method), Stewart [St] obtained in 1977 the same minoration but with a constant $c \neq 1 / 52$ instead of $1 / 52$ (Waldschmidt [W0]). In 1979, Dobrowolski [Do2], using an auxiliary function, obtained the asymptotic minoration

$$
\mathrm{M}(\alpha)>1+(1-\epsilon)\left(\frac{\log \log d}{\log d}\right)^{3}, \quad d>d_{5},
$$

with the constant $1-\epsilon$ replaced by $1 / 1200$ for any $d \geq 2$, for an effective version of the minoration. For sufficiently large degree $d$, Waldschmidt [W2], Theorem 3.17, showed that the constant $1-\epsilon$ could be replaced by $1 / 250$ with a transcendence proof which uses an interpolation determinant. If $\alpha$ is a nonzero algebraic number of degree $d \geq 2$, Voutier [V] obtained the effective minorations :

$$
\mathrm{M}(\alpha)>1+\frac{1}{4}\left(\frac{\log \log d}{\log d}\right)^{3} \quad \text { and } \quad \mathrm{M}(\alpha)>1+\frac{2}{(\log (3 d))^{3}}
$$


For algebraic integers $\alpha$, of degree $d$, of norm $N(\alpha)$, which are totally real, Schinzel's minoration (1.7) was improved by Bertin [Bn] as

$$
\mathrm{M}(\alpha) \geq \max \left\{\theta_{2}^{-d / 2}, \sqrt{|N(\alpha)|} \theta_{2}^{-\frac{d}{2|N(\alpha)|^{1 / d}}}\right\} .
$$

Further, following Bertin [Bn], if a $\nu$-Salem is by definition an algebraic integer $\alpha$ having $\nu$ conjugates outside the closed unit disc and at least one conjugate of modulus 1 , then the measure $\mathrm{M}(\alpha)$ of such an algebraic integer, of degree $2 \nu+2 k$, totally real (i.e., for which the conjugates of modulus $>1$ are all real), satisfies

$$
\mathrm{M}(\alpha) \geq \theta_{2}^{-\frac{\nu}{2^{k / \nu}}} .
$$

Zaimi [Zi] also obtained minorations for the Mahler measures of algebraic integers named $K$-Pisot numbers.

Lehmer's Conjecture is solved in some cases. If $\alpha$ is an algebraic number of degree $d$ such that there exists a prime number $p \leq d \log d$ that is not ramified in the field $\mathbb{Q}(\alpha)$, then Mignotte [Mt0] showed: $\mathrm{M}(\alpha) \geq 1.2$. For any noncyclotomic irreducible polynomial $P$ with all odd coefficients, Borwein, Dobrowolski and Mossinghoff [BDM] showed

$$
\mathrm{M}(P) \geq 5^{1 / 4}=1.4953 \ldots
$$

In terms of the Weil height, Amoroso and David [ADd2] proved that there exists a constant $c>0$ such that, for all nonzero algebraic number $\alpha$, of degree $d$, not being a root of unity, under the assumption that the extension $\mathbb{Q}(\alpha) / \mathbb{Q}$ is Galois, then

$$
h(\alpha) \geq \frac{c}{d} .
$$

If $\mathbb{L} / \mathbb{Q}$ is an abelian extension of number fields, Amoroso and Dvornicich [AD] proved

$$
h(\alpha) \geq \frac{\log 5}{12}
$$

for any nonzero $\alpha \in \mathbb{L}$ which is not a root of unity. Later, given an abelian extension $\mathbb{L} / \mathbb{Q}$ of number fileds and a nonzero algebraic number $\alpha$ which is not a root of unity, with $D:=[\mathbb{L}(\alpha): \mathbb{L}]$, Amoroso and Zannier [AZ1] proved the relative result, which makes use of Dobrowolski's minoration and the previous minoration

$$
h(\alpha) \geq \frac{c(\mathbb{K})}{D}\left(\frac{\log \log 5 D}{\log 2 D}\right)^{13},
$$

where $c(\mathbb{K})>0$. Amoroso and Delsinne [ADn] computed a lower bound, depending upon the degree and the discriminant of the number field $\mathbb{K}$, for the 


\section{JEAN-LOUIS VERGER-GAUGRY}

constant $c(\mathbb{K})$. In 2010 , given $\mathbb{K} / \mathbb{Q}$ an extension of algebraic number fields, of degree $d$, Amoroso and Zannier [AZ2] showed

$$
h(\alpha) \geq 3^{-d^{2}-2 d-6}
$$

for any nonzero algebraic number $\alpha$ which is not a root of unity such that $\mathbb{K}(\alpha) / \mathbb{K}$ is abelian. As a corollary they obtained

$$
h(\alpha) \geq 3^{-14}
$$

for any dihedral extension $\mathbb{L} / \mathbb{Q}$ and any nonzero $\alpha \in \mathbb{L}$ which is not a root of unity. For cyclotomic extensions, they obtained sharper results:

(i) If $\mathbb{K}$ is a number field of degree $d$, there exists an absolute constant $c_{2}>0$ such that, with $\mathbb{L}$ denoting the number field generated by $\mathbb{K}$ and any given root of unity, then

$$
h(\alpha) \geq \frac{c_{2}}{d} \frac{(\log \log 5 d)^{3}}{(\log 2 d)^{4}},
$$

for any nonzero $\alpha \in \mathbb{L}$ which is not a root of unity.

(ii) if $\mathbb{K}$ is a number field of degree $d$, and $\alpha$ any nonzero algebraic number, not a root of unity, such that $\alpha^{n} \in \mathbb{K}$ for some integer $n$ under the assumption that $\mathbb{K}(\alpha) / \mathbb{K}$ is an abelian extension, then

$$
h(\alpha) \geq \frac{c_{3}}{d} \frac{(\log \log 5 d)^{2}}{(\log 2 d)^{4}},
$$

for some constant $c_{3}>0$.

Pr o of of The or em 1.2. We now extend the asymptotic expansions of Section 4.2 with the second-order terms obtained in Section 5.1, inserting them in $(4.75)$.

First, with the new expansions of the moduli of the roots $z_{j, n}$, we have

$$
\begin{aligned}
\sum_{j=\left\lceil v_{n}\right\rceil}^{\lfloor n / 6\rfloor} \log \left|z_{j, n}\right|=\sum_{j=\left\lceil v_{n}\right\rceil}^{\lfloor n / 6\rfloor} \log (1 & +\frac{1}{n} \log \left(2 \sin \left(\frac{\pi j}{n}\right)\right) \\
& \left.+\frac{1}{2 n}\left(\frac{\log \log n}{\log n}\right)^{2}+\frac{1}{n} O\left(\frac{(\log \log n)^{2}}{(\log n)^{3}}\right)\right)
\end{aligned}
$$

with the constant 1 involved in the Big O. Let us apply the remainder Theorem of alternating series: for $x$ real, $|x|<1$ the following inequality holds

$$
|\log (1+x)-x| \leq \frac{x^{2}}{2}
$$


Then

$$
\begin{aligned}
& \left|\sum_{j=\left\lceil v_{n}\right\rceil}^{\lfloor n / 6\rfloor} \log \right| z_{j, n}\left|-\sum_{j=\left\lceil v_{n}\right\rceil}^{\lfloor n / 6\rfloor} \frac{1}{n} \log \left(2 \sin \left(\frac{\pi j}{n}\right)\right)-\sum_{j=\left\lceil v_{n}\right\rceil}^{\lfloor n / 6\rfloor} \frac{1}{2 n}\left(\frac{\log \log n}{\log n}\right)^{2}\right| \\
& \leq \sum_{j=\left\lceil v_{n}\right\rceil}^{\lfloor n / 6\rfloor} \frac{1}{n}\left|O\left(\frac{(\log \log n)^{2}}{(\log n)^{3}}\right)\right| \\
& \quad+\frac{1}{2} \sum_{j=\left\lceil v_{n}\right\rceil}^{\lfloor n / 6\rfloor} \frac{1}{n^{2}}\left[\log \left(2 \sin \left(\frac{\pi j}{n}\right)\right)+\frac{1}{2}\left(\frac{\log \log n}{\log n}\right)^{2}+O\left(\frac{(\log \log n)^{2}}{(\log n)^{3}}\right)\right]^{2} .
\end{aligned}
$$

Similarly as the upper bound in (4.78), we deduce that the upper bound in (5.83) is

Therefore,

$$
O\left(\frac{(\log \log n)^{2}}{(\log n)^{3}}\right) \text {. }
$$

$$
(-2) \sum_{j=\left\lceil v_{n}\right\rceil}^{\lfloor n / 6\rfloor} \log \left|z_{j, n}\right|+\sum_{j=\left\lceil v_{n}\right\rceil}^{\lfloor n / 6\rfloor} \frac{2}{n} \log \left(2 \sin \left(\frac{\pi j}{n}\right)\right)=O\left(\left(\frac{\log \log n}{\log n}\right)^{2}\right)
$$

with the constant $1 / 6$ involved in the Big O. Since $\log \theta_{n}=O\left(\frac{\log n}{n}\right)$ and

$$
\sum_{j=\left\lceil u_{n}\right\rceil}^{\left\lfloor v_{n}\right\rfloor} \log \left|z_{j, n}\right|=O\left(\frac{(\log n)^{2+\epsilon}}{n}\right), \quad \sum_{j=1}^{\left\lfloor u_{n}\right\rfloor} \log \left|z_{j, n}\right|=O\left(\frac{(\log n)^{2}}{n}\right),
$$

we deduce

$$
\log \mathrm{M}\left(G_{n}\right)-\log \Lambda+\left(\log \Lambda+\sum_{j=\left\lceil v_{n}\right\rceil}^{\lfloor n / 6\rfloor} \frac{2}{n} \log \left(2 \sin \left(\frac{\pi j}{n}\right)\right)\right)=O\left(\left(\frac{\log \log n}{\log n}\right)^{2}\right)
$$

with the constant $1 / 6$ involved in the Big O.

In (5.84) the summation $\sum_{j=\left\lceil v_{n}\right\rceil}^{\lfloor n / 6\rfloor}$ can be replaced by $\sum_{j=\lceil\log n\rceil}^{\lfloor n / 6\rfloor}$. Indeed, using Remark 3.3,

$$
\sum_{j=\lceil\log n\rceil}^{\left\lceil v_{n}\right\rceil} \frac{2}{n} \log \left(2 \sin \left(\frac{\pi j}{n}\right)\right)=O\left(\frac{(\log n)^{2+\epsilon}}{n}\right) .
$$

Let us now estimate and give an upper bound of

$$
\left|\frac{-1}{\pi} \int_{0}^{\pi / 3} \log (2 \sin (x / 2)) \mathrm{d} x-\sum_{j=\lceil\log n\rceil}^{\lfloor n / 6\rfloor} \frac{-2}{n} \log \left(2 \sin \left(\frac{\pi j}{n}\right)\right)\right| .
$$




\section{JEAN-LOUIS VERGER-GAUGRY}

In (5.85) the sums are truncated Riemann-Stieltjes sums of $\log \Lambda$, the integral being $\log \Lambda$. Referring to Stoer and Bulirsch ([SB], pp 126-128) we now replace $\log \Lambda$ by an approximate value obtained by integration of an interpolation polynomial by the methods of Newton-Cotes; we just need to know this approximate value up to

$$
O\left(\left(\frac{\log \log n}{\log n}\right)^{2}\right)
$$

Up to

$$
O\left(\left(\frac{\log \log n}{\log n}\right)^{2}\right)
$$

we will show that: (i) an upper bound of (5.85) is $\frac{1}{6 \log n}$, and (ii) the approximate value of $\log \Lambda$ is independent of the integer $m$ (i.e., step length) used in the Newton-Cotes formulas, assuming the weights $\left(\alpha_{q}\right)_{q=0,1, \ldots, m}$ associated with $m$ all positive. Indeed, if $m$ is arbitrarily large, the estimate of the integral should be very good by these methods, ideally exact at the limit $(m "="+\infty)$.

(i) We consider the decomposition of the integration interval as

$$
\left(0, \frac{\pi}{3}\right]=\left(0, \frac{2 \pi\lceil\log n\rceil}{n}\right] \cup\left(\bigcup_{j=\lceil\log n\rceil}^{\lfloor n / 6\rfloor-1}\left[\frac{2 \pi j}{n}, \frac{2 \pi(j+1)}{n}\right]\right) \cup\left[\frac{2 \pi}{n}\left\lfloor\frac{n}{6}\right\rfloor, \frac{\pi}{3}\right]
$$

and proceed by calculating the estimations of

$$
\left|\frac{-1}{\pi} \int_{\frac{2 \pi j}{n}}^{\frac{2 \pi(j+1)}{n}}(\log (2 \sin (x / 2))) \mathrm{d} x-\frac{-2}{n} \log \left(2 \sin \left(\frac{\pi j}{n}\right)\right)\right|
$$

on the intervals $\mathcal{L}_{j}:=\left[\frac{2 \pi j}{n}, \frac{2 \pi(j+1)}{n}\right], j=\lceil\log n\rceil,\lceil\log n\rceil+1, \ldots,\lfloor n / 6\rfloor-1$. On each such $\mathcal{L}_{j}$, the function $f(x)$ is approximated by its interpolation polynomial $P_{m}(x)$, where $m \geq 1$ is the number of subintervals forming an uniform partition of $\mathcal{L}_{j}$ given by

$$
y_{q}=\frac{2 \pi j}{n}+q \frac{2 \pi}{n} \frac{1}{m}, \quad q=0,1, \ldots, m,
$$

of step length $h:=\frac{2 \pi}{n m}$, and $P_{m}$ the interpolating polynomial of degree $m$ or less with

$$
P_{m}\left(y_{q}\right)=f\left(y_{q}\right), \quad \text { for } q=0,1, \ldots, m .
$$

The Newton-Cotes formulas

$$
\int_{\frac{2 \pi j}{n}}^{\frac{2 \pi(j+1)}{n}} P_{m}(x) \mathrm{d} x=h \sum_{q=0}^{m} \alpha_{q} f\left(y_{q}\right)
$$


provide approximate values of $\int_{\frac{2 \pi j}{n}}^{\frac{2 \pi(j+1)}{n}} f(x) \mathrm{d} x$, where the $\alpha_{q}$ are the weights obtained by integrating the Lagrange's interpolation polynomials. Steffensen [Sff] ([SB], p 127) showed that the approximation error may be expressed as follows

$$
\int_{\frac{2 \pi j}{n}}^{\frac{2 \pi(j+1)}{n}} P_{m}(x) \mathrm{d} x-\int_{\frac{2 \pi j}{n}}^{\frac{2 \pi(j+1)}{n}} f(x) \mathrm{d} x=h^{p+1} \cdot K \cdot f^{(p+1)}(\xi), \quad \xi \in \mathcal{L}_{j},
$$

where $p \geq 2$ is an integer related to $m$, and $K$ a constant.

Using [SB], p. 128, and $m=1$, the method being the "Trapezoidal rule", we have:

$$
p=2, \quad K=1 / 12, \quad \alpha_{0}=\alpha_{1}=1 / 2 .
$$

Then (5.86) is estimated by

$$
\begin{aligned}
& \mid \frac{1}{2} \frac{2 \pi}{n}\left[\frac{-1}{\pi} \log \left(2 \sin \left(\frac{\pi j}{n}\right)\right)+\frac{-1}{\pi} \log \left(2 \sin \left(\frac{\pi(j+1)}{n}\right)\right)\right] \\
& =\frac{1}{n}\left|\log \left(2 \sin \left(\frac{\pi j}{n}\right)\right)-\log \left(2 \sin \left(\frac{\pi(j+1)}{n}\right)\right)\right| \\
& =\frac{2 \pi}{n^{2}}\left|\frac{\cos (\xi / 2)}{2 \sin (\xi / 2)}\right| \leq \frac{1}{n} \frac{1}{\log n} \quad \text { for some } \xi \in \mathcal{L}_{j}, \quad \text { for large } n .
\end{aligned}
$$

The (Steffensen's) approximation error " $h^{3} \cdot(1 / 12) \cdot f^{(2)}(\xi)$ " is

$$
\frac{1}{\pi}\left(\frac{2 \pi}{n}\right)^{3} \frac{1}{12}\left|\frac{-1}{4 \sin ^{2}(\xi / 2)}\right| \leq \frac{1}{6 n} \frac{1}{(\log n)^{2}} .
$$

Summing up the contributions of all the intervals $\mathcal{L}_{j}$, we obtain the following upper bound of (5.85)

$$
\left|\frac{-1}{\pi} \int_{0}^{(2 \pi \log n) / n} \log (2 \sin (x / 2)) \mathrm{d} x\right|+\frac{1}{6 \log n} .
$$

with global (Steffensen's) approximation error

$$
\frac{1}{36} \frac{1}{(\log n)^{2}} \text { which is an } O\left(\left(\frac{\log \log n}{\log n}\right)^{2}\right) \text {. }
$$




\section{JEAN-LOUIS VERGER-GAUGRY}

By integrating by parts the integral in (5.88), for large $n$, it is easy to show that this integral is $=O\left(\frac{(\log n)^{2}}{n}\right)$. We deduce the claim.

(ii) Let us show that the upper bound $1 /(6 \log n)$ is independent of the integer $m$ used, once assumed the positivity of the weights $\left(\alpha_{q}\right)_{q=0,1, \ldots, m}$. For $m \geq 1$ fixed, this is merely a consequence of the relation between the weights in the Newton-Cotes formulas. Indeed, we have $\sum_{q=0}^{m} \alpha_{q}=m$, and therefore

$$
\begin{aligned}
\left|\int_{\frac{2 \pi j}{n}}^{\frac{2 \pi(j+1)}{n}} P_{m}(x) \mathrm{d} x-h m f\left(y_{0}\right)\right| & =h\left|\sum_{q=0}^{m} \alpha_{q}\left(f\left(y_{q}\right)-f\left(y_{0}\right)\right)\right| \\
& \leq h\left(\sum_{q=0}^{m}\left|\alpha_{q}\right|\right) \sup _{\xi \in \mathcal{L}_{j}}\left|f^{\prime}(\xi)\right| .
\end{aligned}
$$

Since $h m=\frac{2 \pi}{n}$ and that the inequality $\sup _{\xi \in \mathcal{L}_{j}}\left|f^{\prime}(\xi)\right| \leq\left|f^{\prime}((2 \pi \log n) / n)\right|$ holds uniformly for all $j$, we deduce the same upper bound as in (5.87) for the Trapezoidal rule. Summing up the contributions over all the intervals $\mathcal{L}_{j}$, we obtain the same upper bound (5.88) of (5.85) as before.

As for the (Steffensen's) approximation errors, they make use of the successive derivatives of the function $f(x)=\log (2 \sin (x / 2))$. We have:

$$
f^{\prime}(x)=\frac{\cos (x / 2)}{2 \sin (x / 2)}, \quad f^{\prime \prime}(x)=-\frac{1}{4 \sin ^{2}(x / 2)}, \quad f^{\prime \prime \prime}(x)=\frac{\cos (x / 2)}{4 \sin ^{3}(x / 2)} \cdots
$$

Recursively, it is easy to show that the $q$-th derivative of $f(x), q \geq 1$, is a rational function of the two quantities $\cos (x / 2)$ and $\sin (x / 2)$ with bounded numerator on the interval $(0, \pi / 3]$, and a denominator which is $\sin ^{q}(x / 2)$. For the needs of majoration in the Newton-Cotes formulas over each interval of the collection $\left(\mathcal{L}_{j}\right)$, this denominator takes its smallest value at $\xi=(2 \pi\lceil\log n\rceil) / n$. Therefore, for large $n$, the (Steffensen's) approximation error " $h^{p+1} \cdot K \cdot f^{(p)}(\xi)$ " on one interval $\mathcal{L}_{j}$ is

$$
O\left(\left(\frac{2 \pi}{n m}\right)^{p+1} \cdot K \cdot \frac{n^{p}}{(\pi \log n)^{p}}\right)=O\left(\frac{1}{n(\log n)^{p}}\right) .
$$

By summing up over the intervals $\mathcal{L}_{j}$, we obtain the global (Steffensen's) approximation error $(p \geq 2)$

$$
O\left(\frac{1}{(\log n)^{p}}\right) \text { which is an } O\left(\left(\frac{\log \log n}{\log n}\right)^{2}\right) \text {. }
$$




\subsection{Minoration of the house $\sqrt{\theta_{n}^{-1}}=\theta_{n}^{-1}$, Schinzel-Zassenhaus Conjec- ture and proof of Theorem 1.8}

Denote by $\mathrm{m}_{h}(n)$ the minimum of the houses of the algebraic integers of degree $n$ which are not a root of unity (to avoid ambiguity with the logarithmic Mahler measure, usually denoted by $m$, we use $\mathrm{m}_{h}$ with the subscript $h$ for "houses"). An algebraic integer $\alpha$, of degree $n$, is said extremal if $\alpha=\mathrm{m}_{h}(n)$. An extremal algebraic integer is not necessarily a Perron number.

In 1965 Schinzel and Zassenhaus [SZ] conjectured that

$$
\mathrm{m}_{h}(n) \geq 1+\frac{c_{1}}{n}
$$

for a constant $c_{1}>0$ (i.e.; independent of $n$ ). The first result in this direction they obtained is: when $\alpha \neq 0$ is an algebraic integer of degree $n \geq 2$ which is not a root of unity, then $|\propto|>1+4^{-(s+2)}$, where $2 s$ is the number of nonreal conjugates of $\alpha$.

For a nonreciprocal algebraic integer $\alpha$ of degree $n$, Cassels $[\mathrm{Ca}]$ obtained the inequality

$$
|\alpha|>1+\frac{c_{2}}{n}, \quad \text { with } c_{2}=0.1
$$

Breusch $[\mathrm{Br}]$, independently and previously, showed that $c_{2}=\log (1.179)=$ $0.165 \ldots$ could be taken; Schinzel [Sc1] noticed that the constant $c_{2}=0.2$ could also be taken. Finally, from Smyth's Theorem [Sy1] which claims that $\mathrm{M}(\alpha) \geq \Theta$, Smyth improved the minoration in 1971, with: $c_{2}=\log \Theta=0.2811 \ldots$ On the other hand, Boyd [Bo4] has shown that $c_{2}$ cannot exceed $\frac{3}{2} \log \Theta=0.4217 \ldots$ In 1997 Dubickas [Ds3] showed that $c_{2}=\omega-\epsilon$ with $\omega=0.3096 \ldots$ the smallest root of an equation in the interval $(\log \Theta,+\infty)$, with $\epsilon>0, n_{0}(\epsilon)$ an effective constant, and for all $n>n_{0}(\epsilon)$.

In 1857, for nonzero algebraic integers $\alpha$, Kronecker showed that $\alpha=1$ if and only if $\alpha$ is a root of unity. The sufficient condition was weakened by Blansky and Montgomery [ByM] who showed that $\alpha$, with $\operatorname{deg} \alpha=n$, is a root of unity provided

$$
|\propto| \leq 1+\frac{1}{30 n^{2} \log (6 n)}
$$

Dobrowolsky [Do1] sharpened this condition by: if

$$
|\alpha|<1+\frac{\log n}{6 n^{2}}
$$

then $\alpha$ is a root of unity. 
For algebraic integers $\alpha$, of degree $n$, Dobrowolski [Do2] showed the inequality (1.5) in 1979. Since $\mathrm{M}(\alpha) \leq|\alpha|^{n}$, (1.5) implies

$$
\mid \alpha>1+(1-\epsilon)\left(\frac{\log \log n}{\log n}\right)^{3} \frac{1}{n}, \quad n>n_{3}(\epsilon) .
$$

The constant coefficient $1-\epsilon$ in (5.91) has been improved successively by Cantor and Strauss [CS], and Rausch [Ra], to $2-\epsilon$, Louboutin [Lt], and Meyer [Me], to $\frac{9}{4}-\epsilon$, Voutier [V] to $\frac{1}{4}$, Dubickas [Ds1] to $\frac{64}{\pi^{2}}-\epsilon$. Waldschmidt [W2] showed that this constant $1-\epsilon$ could be replaced by $1 / 250$, with a proof of transcendence using an interpolation determinant.

Dobrowolski's result is effective [Do2]: he showed that the inequality (5.91) is valid for all $n \geq 3$ with $1-\epsilon$ replaced by $1 / 1200$. In 1991, Matveev [Mv] obtained a minoration which revealed to be better for the small degrees: if $\alpha$ is an algebraic integer, not a root of unity, with $\operatorname{deg}(\alpha)=n \geq 2$, then

$$
\mid \alpha \geq \exp \frac{\log \left(n+\frac{1}{2}\right)}{n^{2}} .
$$

In 2007, Rhin and $\mathrm{Wu}$ [RW] verified the conjecture of Schinzel-Zassenhaus up to $n=28$ and improved Matveev's inequality (5.92) to the following: if $\alpha$ is an algebraic integer, not a root of unity, with $\operatorname{deg}(\alpha)=n$, then for $4 \leq n \leq 12$,

$$
\left\lceil=\exp \frac{3 \log \left(\frac{n}{3}\right)}{n^{2}},\right.
$$

and, for $n \geq 13$,

$$
\widetilde{\alpha}=\exp \frac{3 \log \left(\frac{n}{2}\right)}{n^{2}} .
$$

The lower bound (5.92) is better than Voutier's bound

$$
\mathrm{m}_{h}(n) \geq\left(1+\frac{1}{4}\left(\frac{\log \log n}{\log n}\right)^{3}\right)^{1 / n} .
$$

for $n \leq 1434$, and (5.94) is better than (5.95) for $n \leq 6380$. The two numerical values 1434 and 6380 can be improved if Dubickas's constant [Ds1] $\frac{64}{\pi^{2}}-\epsilon$ replaces $1 / 4$ in Voutier's bound (5.95).

For reciprocal nonzero algebraic integers $\alpha$, of degree $n \geq 2$, which are not roots of unity, the lower bounds of $\alpha$ deduced from the Mahler measure $\operatorname{M}(\alpha)$ have a factor 2 in the coefficient since $\mathrm{M}(\alpha) \leq|\alpha|^{n / 2}$ holds, instead of the general inequality: $\mathrm{M}(\alpha) \leq|\alpha|^{n}$. Namely, Dobrowolski's lower bound becomes

$$
\mid \alpha>1+(2-\epsilon)\left(\frac{\log \log n}{\log n}\right)^{3} \frac{1}{n}, \quad n>n_{4}(\epsilon),
$$


the constant $2-\epsilon$ could be replaced by $\frac{9}{2}-\epsilon$ from Louboutin [Lt], or better by Dubickas's constant. Actually, the constant $\frac{64}{\pi^{2}}-\epsilon$ obtained by Dubickas [Ds1] is indifferently valid for reciprocal and nonreciprocal algebraic integers.

For totally real algebraic integers $\alpha$, of degree $n$, either the house $\propto$ lies in $(1,2]$, in which case there exists a root of unity $\omega$ such that $\alpha=\omega+1 / \omega$ from a theorem of Kronecker (1857) [Sy3], or, for $\alpha>2$ and $\alpha \neq 2 \cos (\pi r)$ for some $r \in \mathbb{Q}$, the following lower bound were obtained by Dubickas [Ds2]

$$
\mid \alpha>2+3.8\left(\frac{(\log \log n)^{3}}{(\log n)^{4}}\right) \frac{1}{n}, \quad n>n_{4}(\epsilon),
$$

where the constant 3.8 can be replaced by 4.6 (Dubickas [Ds3]).

In Boyd [Bo4] the following conjectures are formulated:

Conjecture (Lind - Boyd). The smallest Perron number of degree $n \geq 2$ has minimal polynomial:

$$
\begin{array}{rlr}
X^{n}-X-1 & \text { if } \mathrm{n} \not \equiv 3,5 \bmod 6, \\
\left(X^{n+2}-X^{4}-1\right) /\left(X^{2}-X+1\right) & \text { if } \mathrm{n} \equiv 3 \bmod 6, \\
\left(X^{n+2}-X^{2}-1\right) /\left(X^{2}-X+1\right) & \text { if } \mathrm{n} \equiv 5 \bmod 6 .
\end{array}
$$

Conjecture (Boyd).

(i) If $\alpha$ is extremal, then it is always nonreciprocal.

(ii) If $n=3 k$, then the extremal $\alpha$ has minimal polynomial

$$
X^{3 k}+X^{2 k}-1, \quad \text { or } \quad X^{3 k}-X^{2 k}-1 .
$$

(iii) The extremal $\alpha$ of degree $n$ has asymptotically a number of conjugates $\alpha^{(i)}$ outside the closed unit disc equal to

$$
\cong \frac{2}{3} n, \quad n \rightarrow \infty .
$$

Proposition 5.1. Assuming Lind-Boyd's conjecture and Boyd's conjecture true, the Perron number $\theta_{n}^{-1}, n \geq 2$, is not extremal, except if $n=2,3$.

P r o of. First, by Proposition 3.7 (i), the number of conjugates of $\theta_{n}^{-1}, n \geq 2$, lying outside the closed unit disc, is equal to $1+2\lfloor n / 6\rfloor$. Asymptotically it is equal to $n / 3$ and not to $2 n / 3$ as expected from Boyd's conjecture. Second, for the small values of $n$, the minimal polynomials of $\theta_{n}^{-1}, n \geq 2$, are $X^{n}-X^{n-1}-1$ if $n \not \equiv 5 \bmod 6$, and $\left(X^{n}-X^{n-1}-1\right) /\left(X^{2}-X+1\right)$ if $n \equiv 5 \bmod 6($ Section 2$)$. Then, from Lind-Boyd's conjecture, the only cases of extremality are reached for $n=2,3$.

Let $n \neq 2,3$. How far is $\theta_{n}^{-1}$ from extremality? 
Pro of of The or em 1.8. Let $n \geq 2$. From Proposition 3.1 and Lemma 3.2, the Perron number $\theta_{n}^{-1}$ can be expressed as: $\theta_{n}^{-1}=\mathrm{D}\left(\theta_{n}^{-1}\right)+\operatorname{tl}\left(\theta_{n}^{-1}\right)$ with $\operatorname{tl}\left(\theta_{n}^{-1}\right)=\operatorname{tl}\left(\theta_{n}\right)$, and

$$
\mathrm{D}\left(\theta_{n}^{-1}\right)=1+\frac{\log n}{n}\left(1-\lambda_{n}\right)=1+\frac{\log n}{n}\left(1-\frac{\log \log n}{\log n}\left(\frac{1}{1+\frac{1}{\log n}}\right)\right)
$$

so that, for sufficiently large $n$,

$$
\theta_{n}^{-1}>1+\frac{\log n\left(1-\frac{\log \log n}{\log n}\right)}{n} .
$$

From this strict inequality we deduce (1.10); the Schinzel-Zassenhaus lower bound (1.9) is obtained by computing the values of

$$
n\left(\theta_{n}^{-1}-1\right) \quad \text { for } \quad 2 \leq n \leq 200 .
$$

\subsection{Proofs of Theorem 1.3, Corollary 1.4 and Corollary 1.5}

Pro of of The or em 1.3. We will use the method introduced by Smyth and Boyd in [Bo3], Appendix 2, to deduce the expansions (1.4). The case " $n$ odd" is merely a consequence of Appendix 2 in [Bo3], since

$$
\mathrm{M}\left(G_{n}\right)=\mathrm{M}\left(-1+X+X^{n}\right)=\mathrm{M}\left(-1-X-X^{n}\right)=\mathrm{M}\left(1+X+X^{n}\right),
$$

in this case. Let us now assume that $n$ is even. Let $z=e^{i t}$ for $-\pi<t<\pi$. then

$$
\log \left(1+z-z^{n}\right)= \begin{cases}\log (1+z)-\sum_{m=1}^{\infty} \frac{1}{m}\left(\frac{z^{n}}{1+z}\right)^{m} & \text { if }|t|<\frac{2 \pi}{3}, \\ \log \left(-z^{n}\right)-\sum_{m=1}^{\infty} \frac{1}{m}\left(\frac{1+z}{z^{n}}\right)^{m} & \text { if }|t|>\frac{2 \pi}{3} .\end{cases}
$$

We have

$$
\begin{aligned}
\log \mathrm{M}\left(-1+z+z^{n}\right) & =\log \mathrm{M}\left(1-z-z^{n}\right)=\log \mathrm{M}\left(1+z-z^{n}\right) \\
& =\frac{1}{\pi} \int_{0}^{\pi} \log \left|1+e^{i t}-e^{i n t}\right| \mathrm{d} t \\
& =\frac{1}{\pi} \int_{0}^{\pi} \Re\left(\log \left(1+e^{i t}-e^{i n t}\right)\right) \mathrm{d} t
\end{aligned}
$$

and, by Jensen's formula and in Boyd [Bo3, Appendix 1],

$$
\begin{aligned}
\log \mathrm{M}\left(1+z_{1}-z_{2}\right) & =\log \mathrm{M}\left(\max \left\{\left|1+z_{1}\right|, 1\right\}\right) \\
& =\frac{1}{\pi} \int_{0}^{\pi} \log ^{+}\left|1+e^{i t}\right| \mathrm{d} t=\frac{1}{\pi} \int_{0}^{2 \pi / 3} \log \left|1+e^{i t}\right| \mathrm{d} t \\
& =\frac{1}{\pi} \int_{0}^{2 \pi / 3} \Re\left(\log \left(1+e^{i t}\right)\right) \mathrm{d} t .
\end{aligned}
$$


Then the difference $\Delta:=\log \mathrm{M}\left(1+z-z^{n}\right)-\log \mathrm{M}\left(1+z_{1}-z_{2}\right)$ is

with

$$
\Delta=\frac{-1}{\pi} \Re\left[\sum_{m=1}^{\infty} \frac{1}{m}\left(c_{1}(m)+c_{2}(m)\right)\right]
$$

$$
c_{1}(m)=\int_{0}^{2 \pi / 3}\left(1+e^{i t}\right)^{-m} e^{i n m t} \mathrm{~d} t, \quad c_{2}(m)=\int_{2 \pi / 3}^{\pi}\left(1+e^{i t}\right)^{m} e^{-i n m t} \mathrm{~d} t .
$$

Let $\omega=e^{i \pi / 3}$. Using $1+\omega^{2}=\omega$, we now integrate by parts three times. Then

$$
c_{1}(m)=\frac{\omega^{m(2 n-1)}-2^{-m}}{i n m}+\frac{\omega^{-m(1-2 n)+1}-2^{-m-1}}{i n(n m+1)}+O\left(\frac{1}{n^{3} m}\right)
$$

and

$$
c_{2}(m)=\frac{\omega^{m(1-2 n)}}{i n m}+\frac{\omega^{m(1-2 n)+1}}{i n(n m-1)}+O\left(\frac{1}{n^{3} m}\right) .
$$

We deduce

$$
\Re\left(c_{1}(m)+c_{2}(m)\right)=\frac{1}{n^{2} m} \Re[(-i) \omega 2 \cos (m(1-2 n) \pi / 3)]+O\left(\frac{1}{n^{3} m}\right)
$$

and

$$
\Delta=-\frac{\sqrt{3}}{\pi n^{2}} \sum_{m=1}^{\infty} \frac{1}{m^{2}} \cos (m(1-2 n) \pi / 3)+O\left(n^{-5}\right)
$$

Since $\exp (\Delta)=1-\frac{\sqrt{3}}{\pi n^{2}} \sum_{m=1}^{\infty} \frac{1}{m^{2}} \cos (m(1-2 n) \pi / 3)+O\left(n^{-3}\right)$, the coefficient $s(n)$ is

$$
s(n)=-\frac{\sqrt{3}}{\pi} \sum_{m=1}^{\infty} \frac{1}{m^{2}} \cos (m(1-2 n) \pi / 3) .
$$

Obviously, $s(n+6)=s(n)$ for all (even) integer $n \geq 2$, and $s(4)=s(6)$. Denote by

$$
\Phi(z, s, a)=\sum_{m=0}^{\infty} \frac{z^{m}}{(m+a)^{s}}
$$

the Lerch transcendent function [Al] [Ln], and by $\zeta(2)=\sum_{m \geq 1} m^{-2}=\pi^{2} / 6$ the value of the Riemann zeta function at $z=2$. Let us compute $s(4)$ and $s(8)$. For $n=4$,

$$
s(4)=-\frac{\sqrt{3}}{\pi} \sum_{m=1}^{\infty} \frac{1}{m^{2}} \cos (m \pi / 3)
$$


The $\operatorname{sum} \sum_{m=1}^{\infty} \frac{1}{m^{2}} \cos (m \pi / 3)$ is equal to

$$
\begin{array}{r}
\sum_{\substack{m=1 \\
m \in 6 \mathbb{Z}}}^{\infty} \frac{1}{m^{2}}-\sum_{\substack{m=1 \\
m \in 6 \mathbb{Z}+3}}^{\infty} \frac{1}{m^{2}}+\frac{1}{2}\left[\sum_{\substack{m=1 \\
m \in 6 \mathbb{Z}+1}}^{\infty} \frac{1}{m^{2}}+\sum_{\substack{m=1 \\
m \in 6 \mathbb{Z}+5}}^{\infty} \frac{1}{m^{2}}\right]-\frac{1}{2}\left[\sum_{\substack{m=1 \\
m \in 6 \mathbb{Z}+4}}^{\infty} \frac{1}{m^{2}}+\sum_{\substack{m=1 \\
m \in 6 \mathbb{Z}+2}}^{\infty} \frac{1}{m^{2}}\right] \\
=\frac{1}{36}\left(\zeta(2)-\Phi\left(1,2, \frac{1}{2}\right)+\frac{1}{2}\left[\Phi\left(1,2, \frac{1}{6}\right)+\Phi\left(1,2, \frac{5}{6}\right)\right]\right. \\
\left.-\frac{1}{2}\left[\Phi\left(1,2, \frac{1}{3}\right)+\Phi\left(1,2, \frac{2}{3}\right)\right]\right) .
\end{array}
$$

The decomposition

$$
\sum_{m \geq 1} m^{-2}=\pi^{2} / 6=\sum_{\substack{m=1 \\ m \text { even }}}^{\infty} m^{-2}+\sum_{\substack{m=1 \\ m \text { odd }}}^{\infty} m^{-2}
$$

implies $\Phi\left(1,2, \frac{1}{2}\right)=3 \zeta(2)=\pi^{2} / 2$. Then the decomposition

$$
\frac{\pi^{2}}{8}=\sum_{\substack{m=1 \\ m \text { odd }}}^{\infty} \frac{1}{m^{2}}=\sum_{\substack{q=0 \\ q \in 3 \mathbb{Z}+1}}^{\infty} \frac{1}{(2 q+1)^{2}}+\sum_{\substack{q=0 \\ q \in 3 \mathbb{Z}}}^{\infty} \frac{1}{(2 q+1)^{2}}+\sum_{\substack{q=0 \\ q \in 3 \mathbb{Z}+2}}^{\infty} \frac{1}{(2 q+1)^{2}}
$$

implies

$$
\Phi\left(1,2, \frac{1}{6}\right)+\Phi\left(1,2, \frac{5}{6}\right)=36\left(\frac{\pi^{2}}{8}-\frac{1}{36} \Phi\left(1,2, \frac{1}{2}\right)\right)=4 \pi^{2} .
$$

Finally, the decomposition

$$
\frac{\pi^{2}}{6}=\sum_{m \geq 1} \frac{1}{m^{2}}=\sum_{q=0}^{\infty} \frac{1}{(3 q+3)^{2}}+\sum_{q=0}^{\infty} \frac{1}{(3 q+2)^{2}}+\sum_{q=0}^{\infty} \frac{1}{(3 q+1)^{2}}
$$

implies

$$
\Phi\left(1,2, \frac{1}{3}\right)+\Phi\left(1,2, \frac{2}{3}\right)=9\left(\frac{\pi^{2}}{6}-\frac{1}{9} \zeta(2)\right)=\frac{4 \pi^{2}}{3} .
$$

Summing up the contributions, we obtain $s(4)=-\sqrt{3} \pi / 36$.

For $n=8$,

$$
\begin{gathered}
s(8)=-\frac{\sqrt{3}}{\pi} \sum_{m=1}^{\infty} \frac{1}{m^{2}} \cos (m \pi)=-\frac{\sqrt{3}}{\pi}\left(\sum_{\substack{m=1 \\
m^{m} \text { even }}}^{\infty} m^{-2}-\sum_{\substack{m=1 \\
m \text { odd }}}^{\infty} m^{-2}\right) \\
=-\frac{\sqrt{3}}{4 \pi}\left(\zeta(2)-\Phi\left(1,2, \frac{1}{2}\right)\right)=\frac{\sqrt{3} \pi}{12} .
\end{gathered}
$$


Proof of Corollary 1.4. The expansion of $\mathrm{M}\left(G_{n}\right)$ in Theorem 1.3 , is such that the coefficient $s(n)$ is bounded by 1 in modulus for all integers $n \geq 2$, that is uniformly in $n$. We deduce the existence of some integer $n_{0}$ such that $\mathrm{M}\left(G_{n}\right) \geq 1.35$ for all $n \geq n_{0}$ (take $n_{0}=51$ for instance, which corresponds to Figure 1) since $\lim _{m \rightarrow+\infty} \mathrm{M}\left(G_{m}\right)=1.38 \cdots>1.35$. The claim of the uniqueness, for $n=5$, comes from the checking of the values $\mathrm{M}\left(G_{n}\right)$, for all the integers $n$ between 3 and $n_{0}$, by hand and on the computer. The similar claim can also be obtained using (1.3) with a larger value of $n_{0}$ (which underestimates the speed of convergence in some sense).

Pro of of Corollary 1.5. From Theorem 1.3 the function $s(n)$ takes a negative sign in the two cases:

(i) $n \geq 2$ odd and $n \equiv 5(\bmod 6)$; in this case, 3 divides $n+1$ and $\mathrm{M}\left(-1+z+z^{n}\right)=\mathrm{M}\left(1+z+z^{n}\right)<\Lambda$ as soon as $n$ is large enough,

(ii) $n \geq 2$ even and $n \equiv 0$ or $4(\bmod 6)$; in this case, 3 does not divide $n+1$ and $\mathrm{M}\left(-1+z+z^{n}\right)=\mathrm{M}\left(-1-z+z^{n}\right)<\Lambda$ as soon as $n$ is large enough.

The conditions are necessary and sufficient since the coefficients $s(n)$ take positive signs in the other respective cases. In the statement of Smyth's conjecture the trinomials $G_{n}$ only refer to the cases $k=1$ of the first and third items. To finish up the proof of Corollary 1.5 we observe that the coefficient $s(n)$ is uniformly bounded by 1 in modulus for all integers $n \geq 4$. We deduce the existence of some integer $n_{0}$ such that $\mathrm{M}\left(G_{n}\right) \geq 1.35$ for all $n \geq n_{0}$ (take $n_{0}=51$ for instance, as in Figure 1 on the 88), and compute the values of $\mathrm{M}\left(G_{n}\right)$ for $4 \leq n \leq 51$ for comparison with $\Lambda$.

\section{Erdős-Turán-Amoroso-Mignotte Theory and distribution of conjugates}

The purpose of the Erdös-Turán-Amoroso-Mignotte (ETAM) theory is to study the angular regularity of the geometry of the roots of a given polynomial, in sectors all centered at the origin, by establishing best discrepancy functions (Erdős-Turán [ET], Ganelius [G], Amoroso and Mignotte [AM], Mignotte [Mt1] [Mt2]). The ETAM theory is one of the basic ingredient of recent limit equidistribution theorems of conjugates on the unit circle: in Bilu $[\mathrm{Bu}]$, Petsche $[\mathrm{Pe}]$ and Pritsker $[\mathrm{Pr}]$. 


\subsection{Proof of Theorem 1.9}

Let $n \geq n_{0}=18$ and $1 \leq j \leq\left\lfloor\frac{n-1}{4}\right\rfloor$.

(i) First, let us prove (1.12). Let us decompose the summation in (1.12) as

$$
\sum_{j=1}^{\left\lfloor\frac{n-1}{4}\right\rfloor} \arg \left(z_{j, n}\right)=\sum_{j=1}^{\left\lfloor v_{n}\right\rfloor} \arg \left(z_{j, n}\right)+\sum_{j=\left\lceil v_{n}\right\rceil}^{\left\lfloor\frac{n-1}{4}\right\rfloor} \arg \left(z_{j, n}\right) .
$$

Using (3.30), (3.61) and (3.62), the roots $z_{j, n}$ of $G_{n}$ lying in the main Sector " $\frac{\pi}{2}>\arg z>2 \pi \frac{\log n}{n} "$ have the following arguments:

$$
\arg \left(z_{j, n}\right)=2 \pi\left(\frac{j}{n}+\Re\right) \quad \text { with } \quad \Re=-\frac{1}{2 \pi n}\left[\frac{1-\cos \left(\frac{2 \pi j}{n}\right)}{\sin \left(\frac{2 \pi j}{n}\right)} \log \left(2 \sin \left(\frac{\pi j}{n}\right)\right)\right]
$$

with

$$
\operatorname{tl}\left(\arg \left(z_{j, n}\right)\right)=+\frac{1}{n} O\left(\left(\frac{\log \log n}{\log n}\right)^{2}\right)
$$

with the constant $2 \pi$ involved in the Big O. Second, using (3.45), (3.46) and (3.47), the roots which lie in the "Bump Sector", in the first quadrant, are such that

$$
\left|\frac{1}{\left\lfloor\frac{n-1}{4}\right\rfloor} \sum_{j=1}^{\left\lfloor v_{n}\right\rfloor} \arg \left(z_{j, n}\right)\right|=\left|\frac{2 \pi\left(1-\frac{1}{\log n}\right)}{n\left\lfloor\frac{n-1}{4}\right\rfloor} \sum_{j=1}^{\left\lfloor v_{n}\right\rfloor} j+\cdots\right|=O\left(\frac{(\log n)^{2+\epsilon}}{n^{2}}\right) .
$$

Then

$$
\lim _{n \rightarrow+\infty} \frac{1}{\left\lfloor\frac{n-1}{4}\right\rfloor} \sum_{j=1}^{\left\lfloor\frac{n-1}{4}\right\rfloor} \arg \left(z_{j, n}\right)=\lim _{n \rightarrow \infty} \frac{2 \pi}{n\left\lfloor\frac{n-1}{4}\right\rfloor} \sum_{j=\lceil\log n\rceil}^{\left\lfloor\frac{n-1}{4}\right\rfloor} j=\frac{\pi}{4} .
$$

(ii) Now, to prove (1.13), let us observe that the Riemann-Stieljes sum

$$
\begin{aligned}
S(a, n) & :=-\sum_{j=1}^{\lfloor(n-1) / 4\rfloor} \frac{1}{n} \tan \left(\frac{\pi j}{n}\right) \log \left(2 \sin \left(\frac{\pi j}{n}\right)\right) \\
& =\frac{-1}{2 \pi} \sum_{j=1}^{\lfloor(n-1) / 4\rfloor}\left(x_{j}-x_{j-1}\right) a\left(x_{j}\right)
\end{aligned}
$$

with $x_{j}=\frac{2 \pi j}{n}$ and $a(x):=\tan \left(\frac{x}{2}\right) \log \left(2 \sin \left(\frac{x}{2}\right)\right)$ converges to the limit

$$
\lim _{n \rightarrow \infty} S(a, n)=\frac{-1}{2 \pi} \int_{0}^{\pi / 2} a(x) \mathrm{d} x=0.07994 \ldots
$$


To estimate the second-order terms, we proceed as in Subsection 5.2 to give an upper bound of

$\left|\frac{-1}{2 \pi} \int_{0}^{\pi / 2} \tan \left(\frac{x}{2}\right) \log \left(2 \sin \left(\frac{x}{2}\right)\right) \mathrm{d} x-\sum_{j=\lceil\log n\rceil}^{\lfloor(n-1) / 4\rfloor} \frac{-1}{n} \tan \left(\frac{\pi j}{n}\right) \log \left(2 \sin \left(\frac{\pi j}{n}\right)\right)\right|$.

with Newton-Cotes methods applied to the integral. We apply the Trapezoidal rule with the error terms in the tail $\operatorname{tl}\left(\sum \arg \left(z_{j, n}\right)\right)$ controlled by Steffensen's approximation error and $\frac{1}{n} O\left(\left(\frac{\log \log n}{\log n}\right)^{2}\right)$. Let us prove that these terms are here negligible. The derivative

$$
a^{\prime}(x)=\frac{1}{2}\left(1+\frac{\log (2 \sin (x / 2))}{\cos ^{2}(x / 2)}\right) \text { satisfies } \sup _{y \in\left[\frac{2 \pi \log n}{n}, \frac{\pi}{2}\right]}\left|a^{\prime}(y)\right| \leq \log n .
$$

Hence an upper bound of (6.97) is

$$
\frac{n}{4} \frac{1}{2 n} \frac{\pi}{n} \log n+\left|\frac{1}{2 \pi} \int_{0}^{(2 \pi \log n) / n} \tan (x / 2) \log (2 \sin (x / 2)) \mathrm{d} x\right|
$$

which is negligible, as for (5.88) (by integration by parts for the integral). Therefore,

$$
\frac{1}{\left\lfloor\frac{n-1}{4}\right\rfloor} \sum_{j=1}^{\left\lfloor\frac{n-1}{4}\right\rfloor} \arg \left(z_{j, n}\right)-\frac{\pi}{4}=O\left(\frac{1}{n}\left(\frac{\log \log n}{\log n}\right)^{2}\right)
$$

with the constant $2 \pi$ in the Big $\mathrm{O}$. We deduce the claim.

\subsection{An upper bound for Mignotte's discrepancy functions}

Let $\kappa:=\sum_{0}^{\infty} \frac{(-1)^{m-1}}{(2 m+1)^{2}}=0.916 \ldots$ be the constant of Catalan. Denote

$$
C:=\int_{0}^{2 \pi}\left(\int_{0}^{2 \pi} \log ^{+}\left|e^{i t}+e^{i s}-1\right| \frac{\mathrm{d} t}{2 \pi}\right) \frac{\mathrm{d} s}{2 \pi}=0.42627 \ldots
$$

Mignotte [Mt1] [Mt2] and Amoroso and Mignotte [AM] have improved the discrepancy function previously given by Erdős and Turán in [ET]. Let us recall Mignotte's discrepancy function for a polynomial $R$ in $\mathbb{Z}[X][\mathrm{Mt2}]$ : the "radial" operator defined as ${ }^{(r)}: \mathbb{Z}[X] \rightarrow \mathbb{R}[X]$,

$$
R(X)=a_{0} \prod_{j=1}^{m}\left(X-\alpha_{j}\right) \rightarrow R^{(r)}(X)=\prod_{j=1}^{m}\left(X-\frac{\alpha_{j}}{\left|\alpha_{j}\right|}\right),
$$

transforms the polynomial $R(X)$ given in its factored form $(m \geq 1$ being its degree, $a_{0} \neq 0$ its leading coefficient and $\left(\alpha_{i}\right)$ its roots in $\left.\mathbb{C}\right)$ to another 


\section{JEAN-LOUIS VERGER-GAUGRY}

polynomial $R^{(r)}(X)$, which is monic, of the same degree, and has all its roots on $|z|=1$. Mignotte's discrepancy function of the polynomial $R$ is defined by

$$
\widetilde{h}(R)=\frac{1}{2 \pi} \int_{0}^{2 \pi} \log ^{+}\left|R^{(r)}\left(e^{i \theta}\right)\right| \mathrm{d} \theta
$$

and satisfies the inequality, for all $0 \leq a<b \leq 2 \pi$,

$$
\left|\frac{1}{m} \times\left(\sum_{\alpha_{i}, a<\arg \left(\alpha_{i}\right)<b} 1\right)-\frac{b-a}{2 \pi}\right|^{2} \leq \frac{2 \pi}{\kappa} \times \frac{\widetilde{h}(R)}{m} .
$$

Theorem 6.1.

$$
\limsup _{n \rightarrow \infty} \widetilde{h}\left(G_{n}\right) \leq C
$$

P r o o f. On one hand, from Erdős-Turán [ET, p 112, Schur's remark], we deduce the following inequality for the moduli:

$$
\left|G_{n}^{(r)}\left(e^{i \theta}\right)\right| \leq\left|G_{n}\left(e^{i \theta}\right)\right| \text { for all } n \geq 2 \text { on }[0,2 \pi]
$$

On the other hand,

$$
\frac{1}{2 \pi} \int_{0}^{2 \pi} \log ^{+}\left|G_{n}\left(e^{i \theta}\right)\right| \mathrm{d} \theta=\frac{1}{2 \pi} \int_{0}^{2 \pi} \log ^{+}\left|e^{i n t}+e^{i t}-1\right| \mathrm{d} t .
$$

But Lemma 1 in Boyd [Bo2] implies that the following limit holds

$$
\lim _{n \rightarrow+\infty} \frac{1}{2 \pi} \int_{0}^{2 \pi} \log ^{+}\left|e^{i n t}+e^{i t}-1\right| \mathrm{d} t=\int_{0}^{2 \pi}\left(\int_{0}^{2 \pi} \log ^{+}\left|e^{i s}+e^{i t}-1\right| \frac{\mathrm{d} t}{2 \pi}\right) \frac{\mathrm{d} s}{2 \pi}
$$

using bivariate Mahler measures. We deduce (6.100) from the formulas (6.98), (6.101) and (6.102).

As a consequence, we deduce from (6.99) the following inequalities relative to the trinomials $G_{n}$ : for all $\epsilon>0$, for all $0 \leq a<b \leq 2 \pi$, there exists $n_{0}$ such that

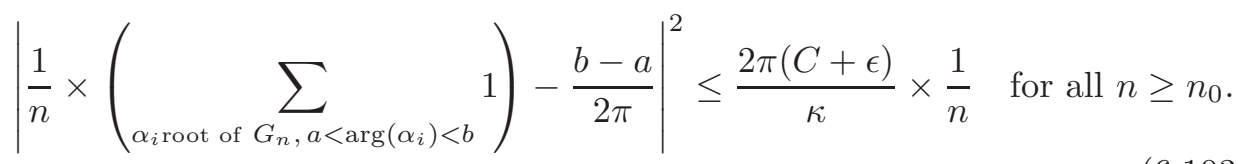

The proof of Theorem 6.1 relies upon the limit properties of bivariate Mahler measures in the method developped by Smyth and Boyd [Bo2]. Comparatively, the method of asymptotic expansions of the roots $\theta_{n}, z_{j, n}$, of $G_{n}$ can also be 
applied directly to obtain the analogue of (6.100) with a constant $C^{\prime} \geq C$ instead of $C$ : it suffices to deduce the asymptotic expansions of the complex numbers $z_{j, n} /\left|z_{j, n}\right|$ of modulus 1 from those of $z_{j, n}$, resp. $\left|z_{j, n}\right|$, i.e.; from Proposition 3.4 and Proposition 3.5, and insert them into the discrepancy functions $\widetilde{h}\left(G_{n}\right)$ of Mignotte (6.98). Though this method works, the upper bound of the limsup in (6.100) is less good. We leave the details of the calculations to the reader.

\subsection{Limit equidistribution of conjugates on the unit circle}

Let $h: \overline{\mathbb{Q}} \rightarrow[0,+\infty)$ denote the logarithmic Weil height (the notation $h$ is traditional and presents no ambiguity with Mignotte's discrepancy function $\widetilde{h}$ ). We denote by $\mathbb{C}^{\times}$, resp. $\mathbb{Q}^{\times}$, the multiplicative group of nonzero elements of $\mathbb{C}$, resp. $\mathbb{Q}$. The unit Dirac measure supported at $\omega \in \mathbb{C}$ is denoted by $\delta_{\omega}$ and $\mu_{\mathbb{T}}$ denotes the Haar measure (unit Borel measure), invariant by rotation, that is supported on the unit circle $\mathbb{T}=\{z \in \mathbb{C}|| z \mid=1\}$, compact subgroup of $\mathbb{C}^{\times}$. Given a point $\alpha \in \overline{\mathbb{Q}}^{\times}$, of degree $d=[\mathbb{Q}(\alpha): \mathbb{Q}]$, we define the unit Borel measure (probability)

$$
\mu_{\alpha}=\frac{1}{d} \sum_{\sigma} \delta_{\sigma(\alpha)}
$$

on $\mathbb{C}^{\times}$, the sum being taken over all $d$ embeddings $\sigma: \mathbb{Q}(\alpha) \rightarrow \mathbb{C}$. A sequence $\left\{\alpha_{k}\right\}$ of points in $\overline{\mathbb{Q}}^{\times}$is said to be strict if any proper algebraic subgroup of $\overline{\mathbb{Q}}^{\times}$ contains $\alpha_{k}$ for only finitely many values of $k$. Theorem 6.2 below is stated as a theorem, but is merely a corollary of Theorem 1.1 in Bilu [Bu], or Theorem 1.2 in Petsche $[\mathrm{Pe}]$.

Theorem 6.2. Let $\left\{\theta_{n}^{-1} \mid n=2,3,4, \ldots\right\}$ be the infinite sequence of Perron numbers in $\mathbb{C}^{\times}$which are the dominant roots of the trinomials $G_{n}^{*}$. Then

$$
\mu_{\theta_{n}^{-1}} \rightarrow \mu_{\mathbb{T}}, \quad n \rightarrow+\infty, \quad \text { weakly }
$$

or equivalently,

$$
\mu_{\theta_{n}} \rightarrow \mu_{\mathbb{T}}, \quad n \rightarrow+\infty, \text { weakly, }
$$

i.e., for all bounded, continuous functions $f: \mathbb{C}^{\times} \rightarrow \mathbb{C}$,

$$
\int f d \mu_{\theta_{n}^{-1}} \rightarrow \int f d \mu_{\mathbb{T}}, \quad n \rightarrow+\infty
$$

Proof. The roots of the trinomials $G_{n}, n \geq n_{0}=18$, all belong to a fixed compact which contains the unit circle, which is the compact annulus

$$
\left\{z \in \mathbb{C}\left|1-\frac{2 \log n_{0}}{n_{0}} \leq\right| z \mid \leq 1+\frac{2 \log 2}{n_{0}}\right\} .
$$




\section{JEAN-LOUIS VERGER-GAUGRY}

The supports of $\mu_{\theta_{n}^{-1}}$ all tend to the unit circle if $n$ tends to infinity, by Lemma 2.1. Let us show that the sequence is strict: by Proposition 2.3, the degree $\operatorname{deg}\left(\theta_{n}\right)$ is respectively equal to $n$ if $n \not \equiv 5 \bmod 6$, and to $n-2$ if $n \equiv 5 \bmod 6$. Hence, $\lim \operatorname{deg}\left(\theta_{n}\right)=+\infty$. The Weil heights are respectively $h\left(\theta_{n}\right)=\frac{1}{n} \times \log \mathrm{M}\left(G_{n}\right)$ if $n \not \equiv 5 \bmod 6$, and $h\left(\theta_{n}\right)=\frac{1}{n-2} \times \log \mathrm{M}\left(G_{n}(X) /\left(X^{2}-X+1\right)\right)$ if $n \equiv 5 \bmod 6$, and can be written (1.14) asymptotically. We have $\lim h\left(\theta_{n}\right)=0$. By Theorem 6.1, Mignotte's discrepancy functions are uniformly bounded. These conditions ensure that Bilu's Theorem [Bu] can be applied.

ACKnowledgement. The author would like to thank G. Rhin and M. Waldschmidt for valuable comments and discussions.

\section{REFERENCES}

[AM] ADLER, R. L.-MARCUS, B.: Topological entropy and equivalence of dynamical systems, Mem. Amer. Math. Soc. 20 (1979), no. 219, iv-84.

[A1] AmOROSO, F.: Sur des polynômes de petites mesures de Mahler, C. R. Acad. Sci. Paris Sér. I Math. 321 (1995), 11-14.

[A2] AMOROSO, F.: Algebraic numbers close to 1: results and methods, in: Number Theory (Tiruchirapalli, India 1996), (V. K. Murty and M. Waldschmidt, Eds.) Amer. Math. Soc., Providence, Contemp. Math. 210 (1998), pp. 305-316.

[ADd1] AMOROSO, F.-DAVID, S.: Le théorème de Dobrowolski en dimension supérieure, C. R. Acad. Sci. paris Sér. I Math. 326 (1998), 1163-1166.

[ADd2] AMOROSO, F.-DAVID, S.: Le problème de Lehmer en dimension supérieure, J. Reine Angew. Math. 513 (1999), 145-179.

[ADn] AMOROSO, F.- DELSINNE, S.: Une minoration relative explicite pour la hauteur dans une extension d'une extension abélienne, Diophantine geometry, CRM Series 4, ed. Norm., Pisa (2007), 1-24.

[AD] AMOROSO, F.-DVORNICICH, R.: A lower bound for the height in abelian extensions, J. Number Theory 80 (2000), 260-272.

[AM] AMOROSO, F.-MIGNOTTE, M.: On the distribution of the roots of polynomials, Ann. Inst. Fourier 46 (1996), 1275-1291.

[AZ1] AMOROSO, F.-ZANNIER, U.: A lower bound for the height in Abelian extensions, J. Number Theory 80 (2000), 260-272.

[AZ2] AMOROSO, F.-ZANNIER, U.: A uniform relative Dobrowolski's lower bound over abelian extensions, Bull. London Math. Soc. 42 (2010), 489-498.

[Al] APOSTOL,T. M.: Zeta and related functions, NIST handbook of mathematical functions, (F. W. F. Olver, D. W. Lozier, R. F. Boisvert and C. W. Clark, Eds.) National Institute of Standards and Technology, Washington, DC, and Cambridge University Press, Cambridge, 2010, 601-616.

[Bk] BAKER, M.: Canonical heights on elliptic curves over abelian extensions, Int. Math. Res. Not. 29 (2003), 1571-1589. 


\section{CONJECTURE OF LEHMER, ASYMPTOTIC EXPANSIONS OF THE MAHLER MEASURE}

[B-S] BERTIN, M. J.-DECOMPS-GUillouX, A.-GRANDET-HUGOT, M.PATHIAUX-DELEFOSSE, M.-SCHREIBER, J. P.: Pisot and Salem Numbers, (with a preface by David W. Boyd.), Birkhaüser Verlag, Basel 1992.

[Bn] BERTIN, M. J.: Quelques résultats nouveaux sur les nombres de Pisot et de Salem, Number Theory in Progress, Vol. I, An Intern. Conf. on Number Theory, org. Stefan Banach Int. Math. Research Center in honor of the 60th birthday of Andrzej Schinzel, Zakopane, Poland, 1997, (K. Győry, H. Iwaniec, J. Urnanowicz, W., Eds.) de Gruyter, Berlin (1999),, pp. 1-10.

[BD] BESSER, A.-DENINGER, C.: p-adic Mahler measures, J. Reine Angew. Math., 517 (1999), 19-50.

[Bu] BILU, Y.: Limit distribution of small points on algebraic tori, Duke Math. J. 89 (1997), 465-476.

[ByM] BLANSKY, P. E.-MONTGOMERY, H. L.: Algebraic integers near the unit circle, Acta Arith. 18 (1971), 355-369.

[Bl] BOREL, E.: Leçons sur les Séries Divergentes, Gauthier-Villars, 2e édition, Paris, 1928.

[BDM] BORWEIN, P.-DOBROWOLSKI, E.-MOSSINGHOFF, M. J.: Lehmer's problem for polynomials with odd coefficients, Ann. of Math. 166 (2007), 347-366.

[BS] BORWEIN, P.-STRAUB, A.: Mahler measures, short walks and log-sine integrals, Theoret. Comput. Sci. 479 (2013), 4-21.

[Bo0] BOYD, D. W.: Pisot numbers and the width of meromorphic functions, privately circulated manuscript (January 1977).

[Bo1] BOYD, D. W.: Variations on a Theme of Kronecker, Canad. Math. Bull. 21 (1978), 129-133.

[Bo2] BOYD, D. W.: Kronecker's Theorem and Lehmer's Problem for polynomials in Several Variables, J. Number Th. 13 (1981), 116-121.

[Bo3] BOYD, D. W.: Speculations concerning the range of Mahler's measure, Canad. Math. Bull. 24 (1981), 453-469.

[Bo4] BOYD, D. W.: The maximal modulus of an algebraic integer, Math. Comp. 45 (1985), 243-249.

[BM] BOYD, D. W.-MOSSInGHOFF, M. J.: Small Limit Points of Mahler's Measure, Exp. Math. 14 (2005), 403-414.

[Br] BREUSCH, R.: On the distribution of the roots of a polynomial with integral coeffcients, Proc. Amer. Math. Soc. 2 (1951), 939-941.

[CS] CANTOR, D. C.-STRAUSS, E. G.: On a conjecture of D.H Lehmer, Acta Arith. 42 (1982/83), 97-100. Correction: ibid. 42 (3) (1983), 327.

[Ca] CASSELS, J.W.S.: On a problem of Schinzel and Zassenhaus, J. Math. Sciences 1 (1966), 1-8.

[CV] CHERN, S.-J.-VAALER, J. D.: The distribution of values of Mahler's measure, J. Reine Angew. Math. 540 (2001), 1-47.

[C] COPSON, E. T.: Asymptotic Expansions, (Reprint of the 1965 original), in: Cambridge Tracts in Math., Vol. 55, Cambridge University Press, Cambridge, 2004.

[DH] DAVID, S.-HINDRY, M.: Minoration de la hauteur de Néron-Tate sur les variétés de type C.M., J. Reine Angew. Math. 529 (2000), 1-74. 


\section{JEAN-LOUIS VERGER-GAUGRY}

[Di] DINGLE, R. B.: Asymptotic Expansions: their Derivation and Interpretation, Academic Press, London-New York, 1973.

[DDs] DIXON, J. D.-DUBICKAS, A.: The values of Mahler Measures, Mathematika 51 (2004), 131-148.

[Do1] DOBROWOLSKI, E.: On the Maximal Modulus of Conjugates of an Algebraic Integer, Bull. Acad. Polon. Sci. Sér. Sci. Math. Astronom. Phys. 26 (1978), 291-292.

[Do2] DOBROWOLSKI, E.: On a question of Lehmer and the number of irreducible factors of a polynomial, Acta Arith. 34 (1979), 391-401.

[De] DOCHE, C.: Zhang-Zagier heights of perturbed polynomials, J. Théor. Nombres Bordeaux 13 (2001), 103-110.

[Ds1] DUBICKAS, A.: On a conjecture of A. Schinzel and H. Zassenhaus, Acta Arith. 63 (1993), 15-20.

[Ds2] DUBICKAS, A.: On algebraic numbers of small measure, Lithuanian Math. J. 35 (1995/1996), 333-342.

[Ds3] DUBICKAS, A.: The maximal conjugate of a non-reciprocal algebraic integer, Lithuanian Math. J. 37 (2) (1997/1998), 129-133.

[Ds4] DUBICKAS, A.: Nonreciprocal algebraic numbers of small measure, Comment. Math. Univ. Carolin. 45 (2004), 693-697.

[Ds5] DUBICKAS, A.: On numbers which are Mahler measures, Monatsh. Math. 141 (2004), 119-126.

[E] ERDÉLYI, A.: Asymptotic Expansions, Dover Publications, New York 1956.

[ET] ERDÖS, P.-TURÁN, P.: On the distribution of roots of polynomials, Ann. Math. $\mathbf{5 1}$ (1950), 105-119.

[F] FLAMMANG, V.: The Mahler measure of trinomials of height 1, J. Aust. Math. Soc. 96 (2014), 231-243.

[FGR] FLAmMANG, V.-GRANDCOLAS, M.-RHIN, G.: Small Salem numbers, in: Number Theory in Progress, Vol. 1, (Zakopane-Kościelisko, 1997), de Gruyter, Berlin (1999), 165-168.

[FLP] L. FLATTO, L.-LAGARIAS, J. C.-POONEN, B.: The zeta function of the betatransformation, Ergod. Th. Dynam. Sys. 14 (1994), 237-266.

[GM] GAlATEAU, A.-MAHÉ, V.: Some consequences of Masser's counting Theorem on Elliptic Curves, (2015).

[G] GANELIUS, T.: Sequences of analytic functions and their zeros, Arkiv Math. 3 (1953), $1-50$.

[HS] HINDRY, M.-SILVERMAN, J.: On Lehmer's conjecture for elliptic curves, in: Séminaire de Théorie des Nombres, Paris 1988/1989, Progress in Math. Vol. 91, Birkhäuser, Paris (1990), pp. 103-116.

[Lg] LANGEVIN, M.: Calculs explicites de constantes de Lehmer, in: Groupe de Travail en Théorie Analytique et Élémentaire des Nombres, 1986/1987, Publ. Math. Orsay, Univ. Paris XI, Orsay, Vol. 88, 1988, pp. 52-68.

[La] LAURENT, M.: Minoration de la hauteur de Néron-Tate, in: Séminaire de Théorie des Nombres Paris 1981/1982, Progress in Math., Vol. 38, Birkhäuser, Paris, 1983, pp. 137-152. 


\section{CONJECTURE OF LEHMER, ASYMPTOTIC EXPANSIONS OF THE MAHLER MEASURE}

[La2] LAURENT, M.: Sur quelques résultats récents de transcendance, Some recent results concerning transcendence, Astérisque, Journées Arithmétiques1989 (Luminy 1991/1992), 209-230.

[Lw] LAWTON, W. M.: A Problem of Boyd Concerning Geometric Means of Polynomials,

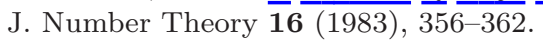

[Le] LEHMER, D. H.: Factorization of certain cyclotomic functions, Ann. Math. 34 (1933), 461-479.

[Ln] LEWIN, L.: Polylogarithms and Associated Functions, with a foreword by A. J. Van der Poorten. North-Holland Publishing Co., New York-Amsterdam, 1981.

[Lt] LOUBOUtin, R.: Sur la mesure de Mahler d'un nombre algébrique, C. R. Acad. Sci. Paris Série I, t. 296 (1983), 707-708.

[Ma] MASSER, D. W.: Counting points of small height on elliptic curves Bull. Soc. Math. France 117 (1989), 247-265.,

[Mv] MATVEEV, E.M.: On the cardinality of algebraic integers, Math. Notes 49 (1991), 437-438.

[Me] MEYER, M.: Le problème de Lehmer, méthode de Dobrowolski et lemme de Siegel "à la Bombieri-Vaaler", Publ. Math. Univ. P. et M. Curie (Paris VI), 90, Problèmes Diophantiens, 1988/1989, No 5 .

[Mt0] MIGNOTTE, M.: Entiers algébriques dont les conjugués sont proches du cercle unité, Séminaire Delange-Pisot-Poitou, 19e année: 1977/78, Théorie des Nombres, Fasc. 2, Exp. No. 39, 6 pp, Paris (1978).

[Mt1] MignOTTE, M.: Sur un théorème de M. Langevin, Acta Arith. 54 (1989), 81-86.

[Mt2] MignOTTE, M.: Remarque sur une question relative à des fonctions conjuguées, C. R. Acad. Sci. Paris, Série I, t. 315 (1992), 907-911.

[Mf] MOSSINGHOFF, M. J.: Polynomials with small Mahler measure, Math. Comp. 67 (1998), 1697-1705, S11-S14.

[MfL] MOSSINGHOFF, M. J.: Known polynomials through degree 180, http://www.cecm.sfu.caz/ mmjm/Lehmer, (1996); implemented (2001): P. Lisonek; and (2003): G. Rhin and J.-M. Sac-Epée; complete through degree 40.

[MRW] MOSSINGHOFF, M. J.-RHIN, G.-WU, Q.: Minimal Mahler Measures, Experimental Math. 17 (2008), 451-458.

[Pe] PETSCHE, C.: A quantitative version of Bilu's equidistribution theorem, Int. J. Number Theory 1 (2005), 281-291.

[P] POINCARÉ, H.: Leçons de Mécanique Céleste Paris, Gauthier-Villars, t. I 1905, t. II-1 1907, t. II-2 1909, t. III 1910.

[Pr] PRITSKER, I. E.: Distribution of algebraic numbers, J. Reine Agew. Math. 657 (2011), 5780 .

[Rz] RATAZZI, N.: Théorème de Dobrowolski-Laurent pour les extensions abéliennes sur une courbe elliptique à multiplications complexes, Int. Math. Res. Not. 58 (2004), 3121-3152.

[Ra] RAUSCH, U.: On a theorem of Dobrowolski about the product of conjugate numbers, Colloq. Math. 50 (1985), 137-142.

[Rd] RÉMOND, G.: Intersection de sous-groupes et de sous-variétés I., Math. Ann. 333 (2005), 525-548. 


\section{JEAN-LOUIS VERGER-GAUGRY}

[Re] RÉNYI, A.: Representations for real numbers and their ergodic properties, Acta Math. Acad. Sci. Hungar. 8 (1957), 477-493.

[RS] RHIN, G.-SMYTH, C. J.: On the absolute Mahler measure of polynomials having all zeros in a sector, Math. Comp. $6 \overline{4(1995)}, \overline{295-304 .}$

[RW] RHIN, G.-WU, Q.: On the absolute Mahler measure of polynomials having all zeros in a sector II, Math. Comp. 74 (2005), 383-388.

[Sc1] SCHINZEL, A.: Reducibility of lacunary polynomials, Acta Arith. 16 (1969), 123-159.

[Sc2] SCHINZEL, A.: On the product of the conjugates outside the unit circle of an algebraic number, Acta Arith. 24 (1973), 385-399; Addendum: ibid. 26 (1974/75), 329-331.

[Sc3] SCHINZEL, A.: On the Mahler measure of polynomials in many variables, Acta Arith. 79 (1997), 77-81.

[SZ] SCHINZEL, A.-ZASSENHAUS, H.: A refinement of two theorems of Kronecker, Michigan Math. J. 12 (1965), 81-85.

[Sr] SELMER, E. S.: On the irreducibility of certain trinomials, Math. Scand. 4 (1956), 287-302.

[Sn] SILVERMAN, J. H.: Lehmer's Conjecture for Polynomials Satisfying a Congruence Divisibility Condition and an Analogue for Elliptic Curves, J. Théorie Nombres Bordeaux 24 (2012), 751-772.

[Si] SINCLAIR, C.: The distribution of Mahler's measures of reciprocal polynomials, Int. J. Math. Math. Sci. 49-52 (2004), 2773-2786.

[Sy1] SMYTH, C.: On the product of the conjugates outside the unit circle of an algebraic integer, Bull. Lond. Math. Soc. 3 (1971), 169-175.

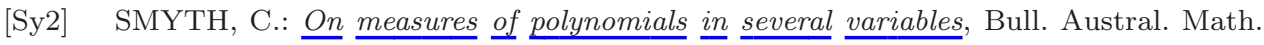
Soc. 23 (1981), 49-63.

[Sy3] SMYTH, C.: The Mahler measure of algebraic numbers: A Survey, in: Number Theory and Polynomials, London Math. Soc. Lecture Note Ser. Vol. 352, Cambridge Univ. press, Cambridge, 2008, pp. 322-349.

[Sy4] SMYTH, C.: Topics in the Theory of Numbers, PhD Thesis, Cambridge 1972.

[Sff] STEFFEnSEN,J. F.: Interpolation, (1927); reprint, 2nd ed. Chelsea Publ. Co, New York 1950.

[St] STEWART, C. L.: Algebraic integers whose conjugates lie near the unit circle, Bull. Soc. Math. France 106 (1978), 169-176.

[SB] StOER, J.-BUliRSCH, R.: , Introduction to Numerical Analysis, in: Texts in Appl. Math. Vol. 12, 2nd ed., Springer-Verlag, New York, 1993.

[VG] VERGER-GAUGRY, J.-L.: Uniform distribution of the Galois conjugates and betaconjugates of a Parry number near the unit circle and dichotomy of Perron numbers, Unif. Distrib. Theory 3 (2008), 157-190.

[V] VOUTIER, P. M.: An effective lower bound for the height of algebraic numbers, Acta Arith. 74 (1996), 81-95.

[W0] WALDSCHMIDT, M.: Sur le produit des conjugués extérieurs au cercle, L'Enseign. Math. 26 (1980), 201-209.

[W1] WALDSCHMIDT, M.: Auxiliary functions in transcendental number theory, SASTRA Ramanujan Lectures, Ramanujan J. 20 no. 3, (2009), 341-373. 


\section{CONJECTURE OF LEHMER, ASYMPTOTIC EXPANSIONS OF THE MAHLER MEASURE}

[W2] WALDSCHMIDT, M.: Diophantine Approximation on Luinear Algebraic Group: Transcendence Properties of the Exponential Function in Several Variables, Grund. Math. Wiss., Vol. 326, Springer-Verlag, Berlin, 2000.

[Wu] WU, Q.: The smallest Perron numbers, Math. Comp. 79 (2010), 2387-2394.

[Za] ZAGIER, D.: Algebraic numbers close to 0 and 1, Math. Comp. 61 (1993), 485-491.

[Zi] ZAÏMI, T.: Sur les K-nombres de Pisot de petite mesure, Acta Arith. 77 (1996), 103-131.

Received March 20, 2015

Accepted September 14, 2015

\author{
Jean-Louis Verger-Gaugry \\ LAMA, CNRS UMR 5127 \\ Université Savoie Mont Blanc \\ 73376 - Le Bourget-du-Lac \\ FRANCE \\ E-mail: Jean-Louis.Verger-Gaugry@univ-smb.fr
}

